\title{
IMPROVING THE SOFTWARE INSPECTION PROCESS WITH PATTERNS
}

Faculty of Science, Department of Information

Processing Science, University of Oulu

OULU 2005

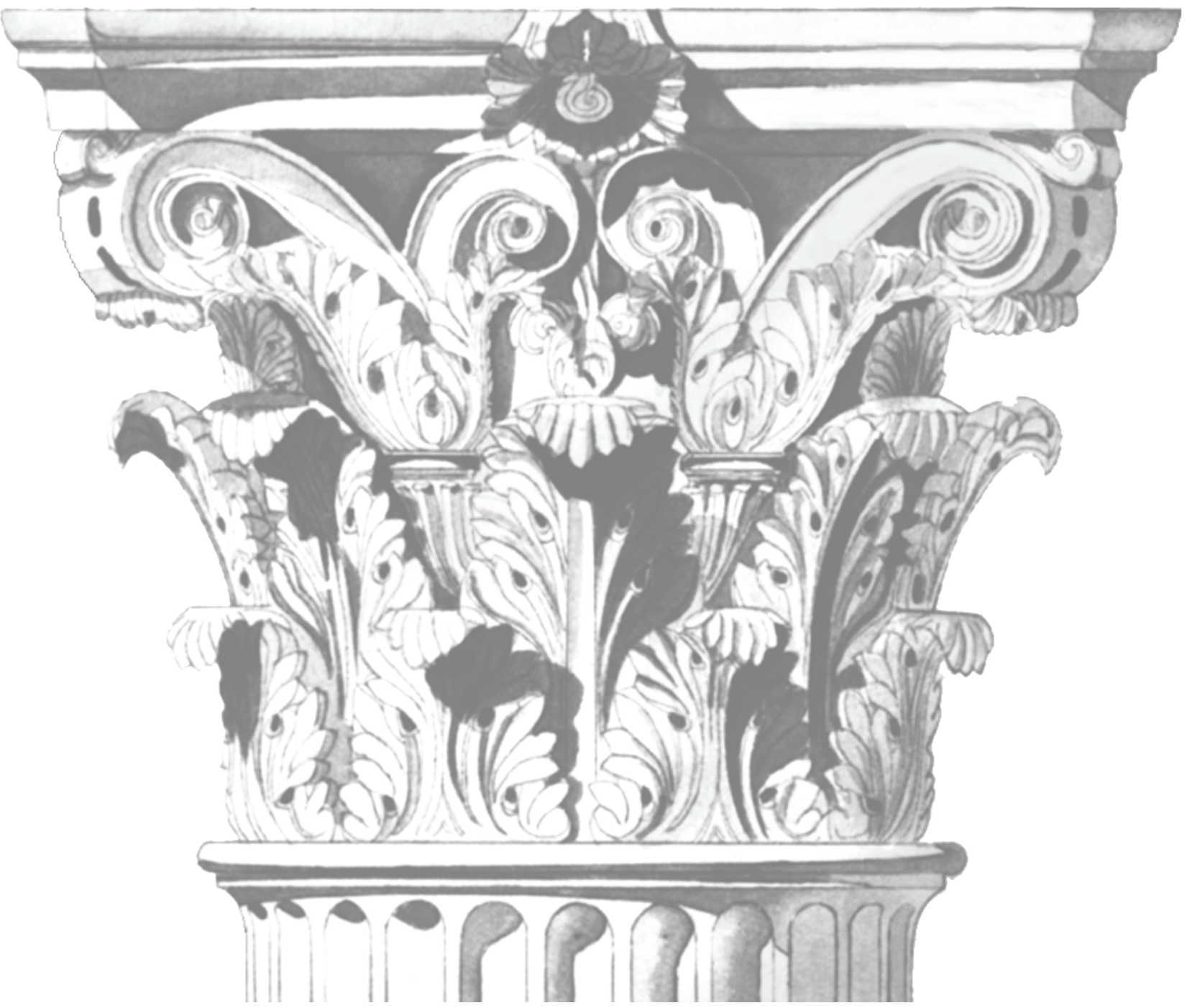





\section{IMPROVING THE SOFTWARE} INSPECTION PROCESS WITH PATTERNS

Academic Dissertation to be presented with the assent of the Faculty of Science, University of Oulu, for public discussion in Auditorium ITI I5, Linnanmaa, on December 16th, 2005, at 12 noon 
Copyright () 2005

University of Oulu, 2005

Supervised by

Professor llkka Tervonen

Reviewed by

Professor Cornelia Boldyreff

Professor Claes Wohlin

ISBN 95I-42-7893-3 (nid.)

ISBN 95I-42-7894-I (PDF) http://herkules.oulu.fi/isbn95 I427894I/

ISSN 0355-319|

http://herkules.oulu.fi/issn03553191/

OULU UNIVERSITY PRESS

OULU 2005 
Harjumaa, Lasse, Improving the software inspection process with patterns

Faculty of Science, Department of Information Processing Science, University of Oulu, P.O.Box 3000, FIN-90014 University of Oulu, Finland

2005

Oulu, Finland

\begin{abstract}
The quality of a software product depends largely on the quality of the process that is used to develop it. In small software companies, the development process may be informal or even ad hoc, which causes uncertainty and variation in the product quality. However, quality issues are as important in small companies as in their larger counterparts. To sustain their dynamics and competitiveness, small organizations need to concentrate on the most effective quality assurance methods.

Software inspection is a proven method for improving product quality and it provides a very costeffective way for small companies to improve their development processes. This study introduces a framework for adjusting the inspection process for the organization's specific needs and evaluating its capabilities. The main focus of this work, however, is on refining and improving the inspection process. The improvement is guided by concrete instructions that are documented as process patterns. The pattern approach has already been used successfully in several other areas of software engineering. Patterns aim at capturing the best practices of software development and transferring this knowledge between people or organizations.

The framework for inspection process capability originates from the literature relating to different types of peer review methods and experiments with flexible and tool-supported inspections in small companies. Furthermore, generic process improvement models are studied to find a feasible structure for the framework. As a result of the analysis, the i3 capability model is introduced. The feasibility of the model has been investigated in real-life software organizations carrying out inspections.

After the capability evaluation, the inspection process can be upgraded with the aid of improvement patterns, which provide structured and easy-to-follow guidelines for implementing improvements. An initial list of patterns, describing solutions to the most common problems confronted in the establishment of inspections, is extracted from related inspection research and an industrial experiment.

The contributions of this study are, first, the new view of the inspection process, based on the fundamental activities that are performed during an inspection instead of a series of stages, as it is usually presented. An activity-based process description enables tailoring of the process for organization-specific needs and its targeted improvement. Second, the study introduces a practical, lightweight method for implementing the improvement. Patterns are especially suitable in companies where resources are limited and full-scale improvement programmes cannot be initiated. Furthermore, the generic process improvement models do not provide detailed information on how improvements should be carried out, and the pattern approach represents a promising method for that. Third, the inspection process currently does not have a very significant role in generic software process improvement models; this study helps in outlining the importance of inspections. A similar approach could be applied to other software subprocesses to enable their evaluation and improvement.
\end{abstract}

Keywords: peer review, process pattern, software inspection, software process improvement, software quality 



\section{Preface}

This thesis summarises the results of the research I have been involved in since I started my postgraduate studies and work in the Department of Information Processing Science. The focus of the research has changed a bit during these past years, but little by little the thesis has shaped up into its current form to cover the process improvement aspect of software inspections.

I wish to thank all the students that have contributed to my study in laboratory experiments and university courses. The help from people in our partner companies has also been invaluable. Especially I would like to thank Pekka Vuorio and Anna Huttunen for their research effort and also for co-authoring some of the papers.

Professor Claes Wohlin and Professor Cornelia Boldyreff, the reviewers of my thesis, have provided very constructive critique to my research. Their feedback and encouragement have ascertained a solid ground for the final version of this thesis.

I am very grateful to my supervisor, Professor Ilkka Tervonen, for his patience and support along the way. His role in supporting my research, postgraduate studies and career has been priceless. He has also been a co-author in many of my articles.

My colleagues deserve sincere thanks for keeping our team's good spirits up. Henrik Hedberg, Juha Iisakka and Jouni Kokkoniemi have formed a research group that has been very enjoyable to work with.

This study has been financially supported by the Tauno Tönning Foundation. The research projects involved have been funded by the Academy of Finland, as well as TEKES and partner companies Nokia Telecommunications FSG, Rautaruukki Tietotekniikka, Sonera Ltd and MSG Software Oy.

I am also grateful to the Department of Information Processing Science for providing me the facilities for the research. I also thank the publishers of the original papers for giving me the permission to reprint the articles as a part of my dissertation.

I wish to thank all my friends and especially Marko for his friendship and approximately a thousand cheerful coffee breaks. Kalervo and Päivi have also been a great source of joy and inspiration for me. I am also thankful for all other wonderful people who have supported me during these years. 
A special thank goes to my parents, Eila and Kauko, and my brother Teemu. Their enduring and unconditional support has always helped me and given me motivation to carry on.

Finally, I wish to express my loving thanks to Marja for her encouragement and support during the preparation of this thesis. You have given me the energy to get this work done.

Oulu, December 2005

Lasse Harjumaa 


\section{List of Original Publications}

I Harjumaa L \& Tervonen I (1998) A WWW-based Tool for Software Inspection. Proceedings of the Thirty-First Hawaii International Conference on System Sciences (HICSS-98), vol III, Kohala Coast, Hawaii: 379-388.

II Harjumaa L \& Hedberg H (2002) Web-based Software Inspection Tools - Past, Present and Future. Proceedings of the 20th IASTED International Multi-Conference Applied Informatics (AI-02), Innsbruck, Austria: 283-288.

III Tervonen I, Iisakka J \& Harjumaa L (2001) A Tailored Capability Model for Inspection Process Improvement. Proceedings of the Second Asia-Pacific Conference on Quality Software (APAQS-01), Hong Kong: 275-282.

IV Harjumaa L, Tervonen I \& Vuorio P (2004) Using Software Inspection as a Catalyst for SPI in a Small Company. Proceedings of the Fifth International Conference of Product Focused Software Process Improvement (PROFES-04), Kansai Science City, Japan: 62-75.

V Harjumaa L, Tervonen I \& Vuorio P (2004) Improving Software Inspection Process with Patterns. Proceedings of the Fourth International Conference on Quality Software (QSIC-04), Braunschweig, Germany: 118-125.

VI Tervonen I \& Harjumaa L (2004) Encouraging SME software companies to adopt software reviews in their entirety. Proceedings of the 22nd Pacific Northwest Software Quality Conference (PNSQC-04), Portland, OR, USA: 525-536.

VII Harjumaa L (2006) A Pattern Approach to Software Inspection Process Improvement. To be published in Software Process Improvement and Practice (accepted for publication 19.4.2005). 



\section{Contents}

Abstract
Preface
List of Original Publications
Contents
1 Introduction


3.5 Requirements for Software Inspection Capability Model.....................................63

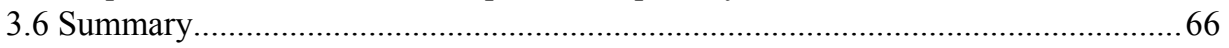

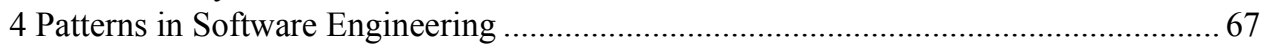

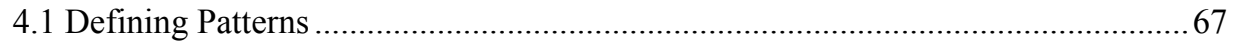

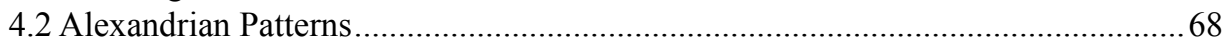

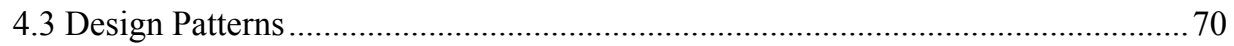

4.4 Examples of Other Software Development Patterns............................................. 71

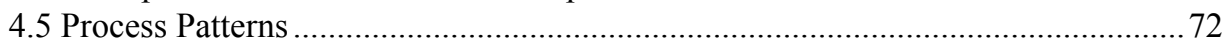

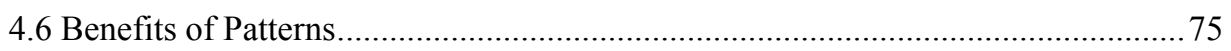

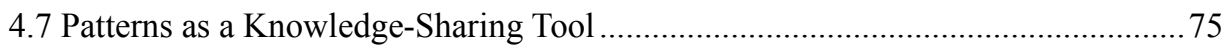

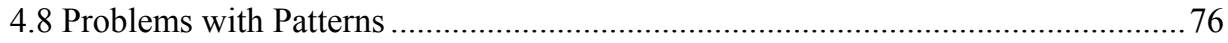

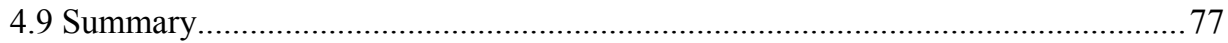

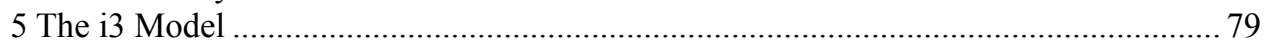

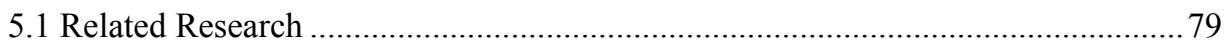

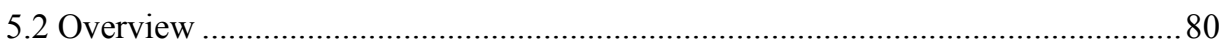

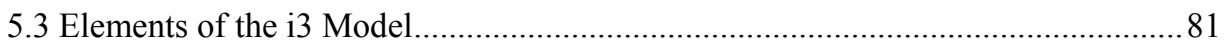

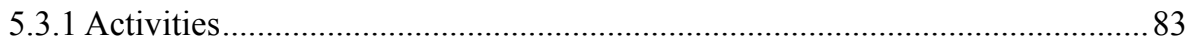

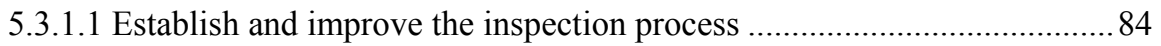

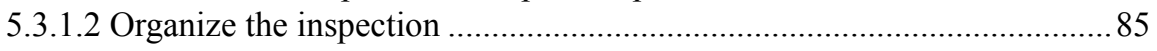

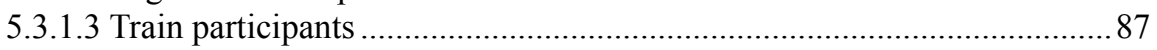

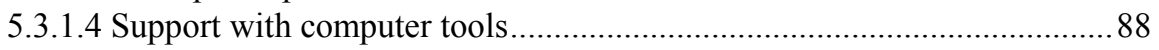

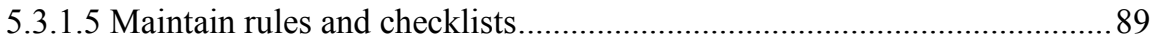

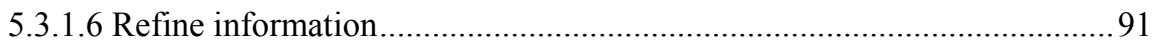

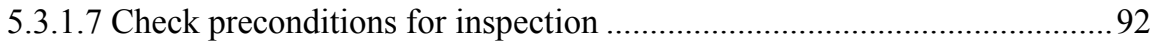

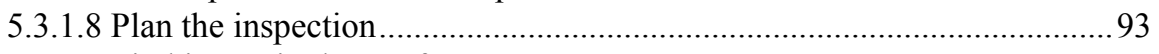

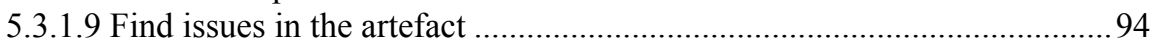

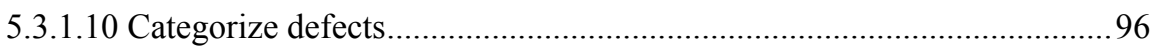

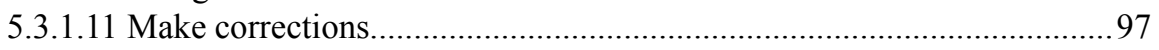

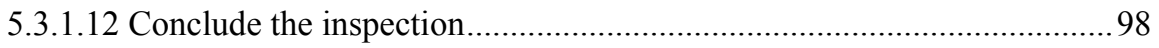

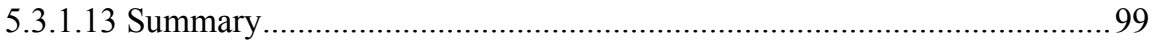

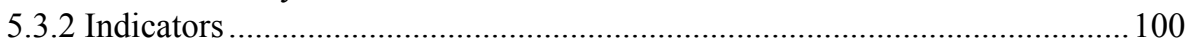

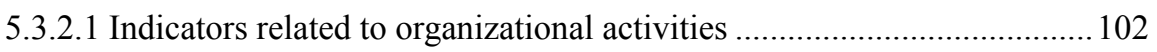

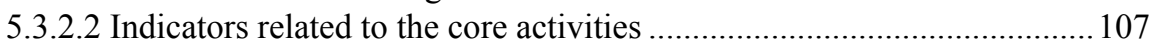

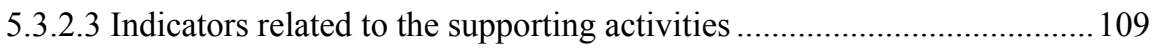

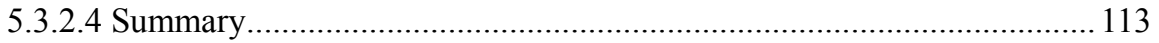

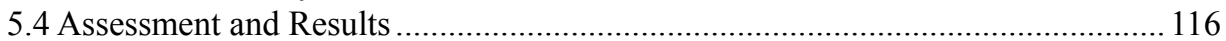

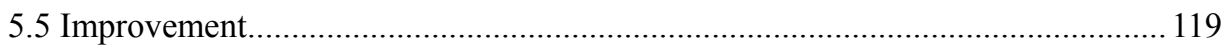

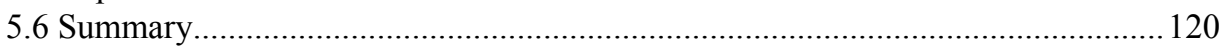

6 The Pattern Approach to Inspection Improvement ................................................... 121

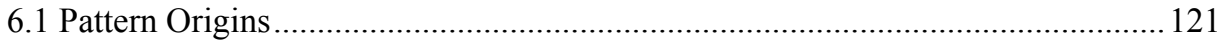

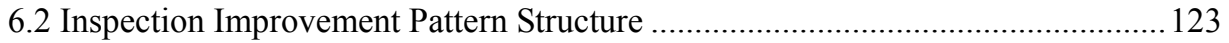

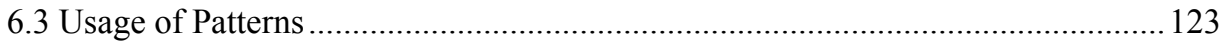

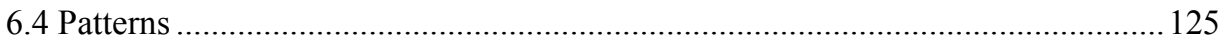

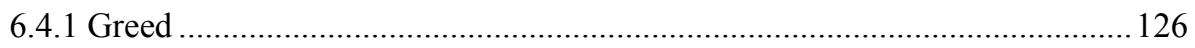

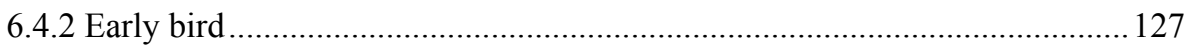

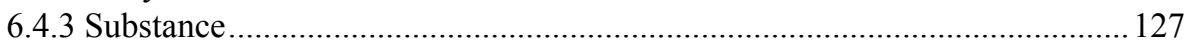




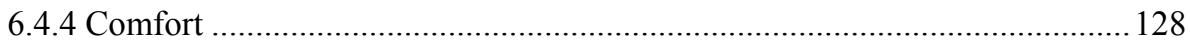

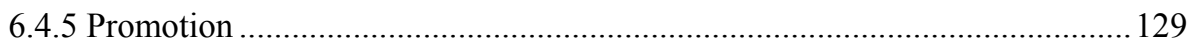

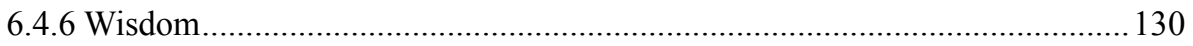

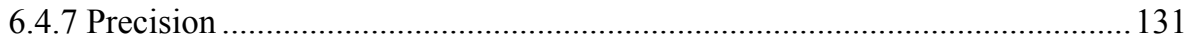

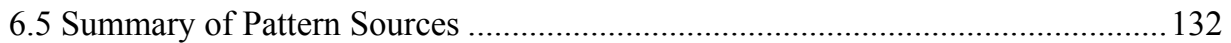

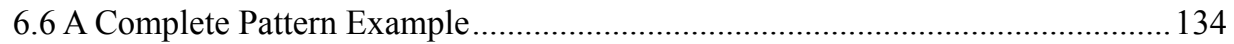

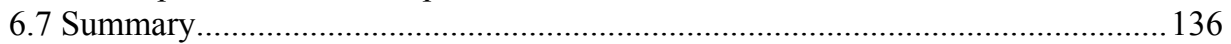

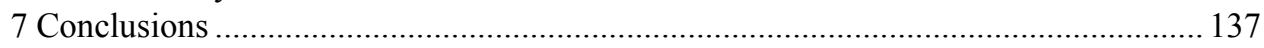

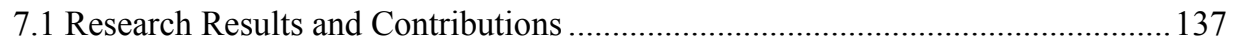

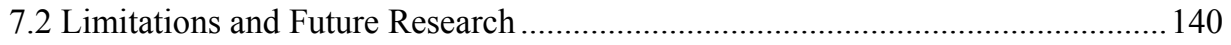

References

Appendix 



\section{Introduction}

Software is nowadays the major component in computer systems. Software controls, for example, our communication, transportation, economic exchanges, health monitoring and entertainment. Software defects and malfunctions can be extremely expensive, in terms of both money and reputation. To an increasing extent even human lives are dependent on software. Practically all everyday routines, from simple office tasks to complicated business transactions rely on software systems. At the same time, building such systems has become more challenging than ever. Development times have to be short, the systems may have interfaces with complex subsystems and other dependencies, yet the software has to be of high quality to be competitive.

Disastrous examples, such as the vanishing of the Mars Climate Orbiter (NASA 1999) and abandoned London stock exchange project Taurus (Drummond 1996), that are attributed to software and have caused financial losses of billions of dollars, are frequently elicited in the literature. An interesting phase of recent computer science history is the $\mathrm{Y} 2 \mathrm{~K}$ problem. It had a minimal effect, but analysing, planning and carrying out the changes necessary to avoid the problem cost billions and billions of dollars around the world.

There are other examples of the importance of software quality. Viruses and worms spreading through the Internet exploit well-known software vulnerabilities, and have caused enormous financial losses. Vulnerabilities and deficiencies exist in software because developers have not devoted adequate efforts to applying quality practices in their work. Harder to measure, but just as severe, are the usability problems caused by poor software design and implementation. These nibble away at people's productivity little by little, every day.

All these examples indicate that quality needs to be a key driving force in any organization dealing with software. Customers have high expectations regarding the usability, reliability and overall quality of products. Even minor irritations caused to the customer may result in the loss of credibility of the provider company. However, there is no single simple procedure to ensure quality (Brooks 1975, Brooks 1987). Instead, continuous and incremental improvement of methodologies, tools and practices in controllable steps is recommended.

It is widely accepted that achieving high quality software products requires a precisely defined process to be followed in the production of the software. The process description 
usually outlines the actions that have to be taken to create a product and to ensure its quality. A process is a collection of procedures, involving a set of tools, techniques and resources that are used to produce the software product. Even if the process is not explicitly defined in an organization, the tasks that are needed to complete a product are usually performed in the same order every time. An explicitly defined process is, however, even more beneficial, ensuring a certain level of consistency in the implementation activities. Processes also structure the development tasks in order to examine, understand, manage and improve the activities.

Several process models have been introduced during the history of software engineering. Perhaps the most widely known is the waterfall model, which describes development at a high-level as stages that follow each other. The stages cover the whole development cycle from the elicitation of requirements to the operation and maintenance phase (Royce 1970). The waterfall model is a typical prescriptive process model, depicting how development should progress. Another famous example of a software process is prototyping, in which a system is built in parts, allowing the developers and the customer to demonstrate the characteristics of the system and assure that they have a common understanding of it.

All process models aim at managing the risk involved in software development and improving the quality of the product being produced. A high-level software development process can also be divided into subprocesses, such as requirements analysis, configuration management, and testing. Some of these processes, such as the inspection process, are supposed to have direct positive implications for product quality. Other processes have an indirect effect on product quality. For example, project management helps us to control the development as a whole.

The processes that are utilized have to be effective and efficient. They need to be tailored for the specific needs and goals of the organization, and when they are running, they should be analysed to increase understanding of the factors that make a software project succeed or fail. There are several methods available for software process evaluation and improvement, one of the first being the Capability Maturity Model (CMM), introduced in the 1980s. Over time, the concept of process maturity evolved, and measurement and improvement became essential elements of effective software processes. Like the actual processes, measurement and improvement can be performed in many different ways.

Design and code reviews and inspections are recommended quality assurance instruments in nearly all process models. While it is widely agreed that process descriptions and reviews or inspections are necessary to improve the quality of the software, they are implemented in greatly varying ways (Aurum et al. 2002). Wrongly practiced processes can result in major declines in productivity and in the motivation of personnel (Shirey 1992).

It is particularly important for small and medium-sized software companies to provide innovative products and services to lead the field, over their competitors. In addition to a sound vision and knowledge of the business domain, this requires a decent strategy for product development. A well-defined software development infrastructure enables the company to focus on quality issues in order to ensure high-quality products and customer satisfaction. Quality has to be built into the products, thus it is important to take it into consideration in every phase of the development cycle. 
Producing reliable and high-quality software is not a trivial task, however. Small and medium sized (SME) companies often operate on the leading-edge, and have limited resources for development. Ensuring an adequate level of quality helps in directing those resources correctly, eliminating gratuitous administrative and corrective work. Building the software on time and on budget is even more important than in larger companies.

Many SME companies still do not have quality assurance functions installed, or quality matters are the responsibility of a single person, who rarely has real opportunities to control and manage the development process adequately in order to provide any practical help towards achieving quality.

Small and medium sized companies are important actors in the software industry. According to Sitra (2001) they employ 9000 people in Finland, 50 percent of the total software engineering resources in the market. These companies form the largest part of the software development and maintenance sectors in many other countries, as well. Annala (2002) has studied 116 young Finnish software companies, and only 10 percent of those studied had more than ten employees. Typically the innovator works alone or with just a few developers in the company's early stages.

To be considered a serious competitor in the software market, SME companies must adopt process practices that are internationally accepted. It is clear that a small organization cannot implement a full-scale software process improvement (SPI) programme at once, but it can still benefit from ideas provided by different improvement models. Certain improvements can even be achieved more easily in minor companies than in their larger counterparts. For example, organising training sessions and ensuring developer involvement is effortless when there are a small number of people in the organization (Guerrero \& Eterovic 2004).

To sustain their dynamics and competitiveness, SMEs need to concentrate on the most effective quality assurance methods. The most beneficial parts of large-scale improvement programmes have to be selected and implemented. The selection depends largely on the type of organization, and its policies and business goals.

As there is no viable automated method for ensuring the correctness of software designs and implementations, old-fashioned human work is needed in verifying and validation of software products. There are currently two efficient methods for verifying and validating the quality of a software artefact - testing and reviewing - which play a vital role in professional software development. Both techniques are exceedingly useful and complementary. Testing catches many errors in a completed piece of code and proves that it runs correctly when given a variety of inputs, and reviews can prevent lapses in software documentation from developing into bugs, even at the early stages of the development cycle (Tian 2001).

Software inspection is the most rigorous type of review. For almost three decades, inspection processes have been the subject of an active research field, and have proven to be an exceptionally cost-effective method for ensuring software quality. The inspection method for software code was first introduced by Michael Fagan in 1976, and is based on collegial evaluation of a document.

In software inspection, a design, code or other piece of documentation is read through carefully by colleagues, who try to identify possible errors in the artefact. The essence of the method is very simple, yet evidence shows that inspections are very useful (Fagan 1976, Gilb \& Graham 1993). There are several techniques that improve the efficiency 
even further: checklists and reading techniques provide guidance as to what types of errors to look for; source documents enable the origins of defects to be tracked; and automated tools provide fast, standardized ways to report and categorize defects.

To be efficient, inspections require certain conditions. Adequate human resources have to be available, and the scheduling has to be carefully planned. The method can be adapted and modified, but an adequate level of rigor has to be maintained when performing inspections; thus the reviewing procedures and tasks defined need to be carried out methodically.

Despite the vast amount of positive evidence, the inspection process is still not practiced generally, and the search for new inspection tools and procedures still continues. Inspections may be considered burdensome in minor companies and not carried out as rigorously as described in textbooks, or perhaps not included in the development process at all. Looking for more convenient forms of inspection and presenting less labour-intensive alternatives would help the companies to adopt the process.

Because most software companies in Finland are quite small and have limited resources, they cannot afford unsuccessful process try-outs. Investments that they make in quality assurance should produce the required results and have real value. Furthermore, small companies do not have the opportunity to endlessly fine-tune their processes in internal improvement projects. Thus, small software companies need methods that can be installed easily, have been empirically tested and found efficient, and produce the desired results quickly.

Combining the elements of software inspection and process improvement may be beneficial for companies with limited resources. However, introducing inspections or a software process improvement programme is expensive. Creating a lightweight method for improving the quality of software development through inspections would enable rapid and cost-effective SPI.

\subsection{Research Problems}

In this thesis, the essence of the inspection process and practical methods for upgrading it within small and medium size companies are sought. The research problem of this thesis can be summarized as follows:

How can the software inspection process be improved with the aid of patterns?

In order to answer this question, the problem needs to be dismantled into a more manageable form. In particular, the problem can be divided into the following subproblems:

\section{How should the effectiveness of the inspection process be evaluated?}

Answering this question provides the basis for the improvement model suggested in this study. Analysing different reviewing techniques and identifying their key elements is crucial to creating a comprehensive model of the activities and work products that are 
involved with inspections. After determining the essence of the inspection process, techniques for assessing the capability of the process can be established.

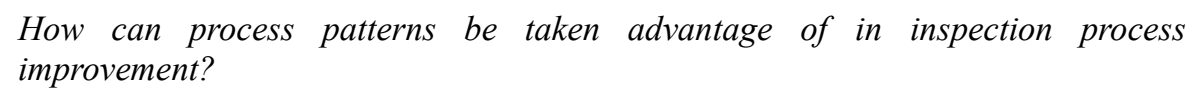

Studying the full-scale improvement models and making use of the results and lessons gained from SPI research helps in creating an effective and flexible improvement model for the inspection process. It is especially interesting to investigate SPI experiences in small companies.

The concept of "patterns" is almost overused in software engineering after their extraordinary success in the field of design patterns. However, it is reasonable to presume that patterns that are very practical and straightforward guidelines will provide an applicable format for offering guidance for improvement to those who have limited resources or knowledge for the implementation of a full-scale improvement programme.

\subsection{Research Approach}

This study includes both conceptual analysis and empirical observations. As a whole, the problem is approached with a constructive research method. This approach has been chosen in order to find solutions for real-life process improvement problems and produce research results which are relevant in practice. The constructive approach is well-suited to this task (March \& Smith 1995, Lukka 2003).

In the constructive research method, attempts are made to solve a real life problem by producing innovative artefacts. The artefact and its production are not ends in themselves, but instead an artefact is used to evaluate the feasibility or functionality of some new idea. In the most general case, the relevance of the idea can be demonstrated. The core concept, the construct, is an abstract concept with an almost unlimited number of varying yet acceptable interpretations. Artefacts, such as models, diagrams, designs and even commercial products, are constructs. It should be pointed out that a construct is not "found", but invented and built (Lukka 2003).

The constructive research method requires that the problem to be solved is closely connected to practice and originates from the real world, but at the same time it has to be carefully connected to previous theoretical knowledge. The practicability of the artefact has to be tested, and this requires close cooperation between the researcher and real world practitioners. Special attention has to be paid to ensuring that the empiric results are reflected in the theoretical framework (Lukka 2003, March \& Smith 1995). Figure 1 illustrates the contributions of this research from theoretical and practical viewpoints. The philosophical background theory of constructive research is pragmatism, which stresses the practical meaningfulness of theories and proposes that theories should be tested and verified (Peirce 1931). The driving force for this research is to provide small software companies with a workable methodology with which to improve their development processes. Traditional process improvement programmes typically require a considerable amount of human and financial resources, which means that small companies have very limited opportunities to accomplish full-scale improvement programmes. The new 
approach is achieved by combining previous knowledge from software inspection writings and research, software process improvement research and pattern studies. Experiments are carried out in real-world development organizations, and with students. The validated results of the study can be reflected in software process improvement theories, as the proposed methodology offers new ideas for SPI. In addition, new ideas and instruments for traditional inspection processes are presented.

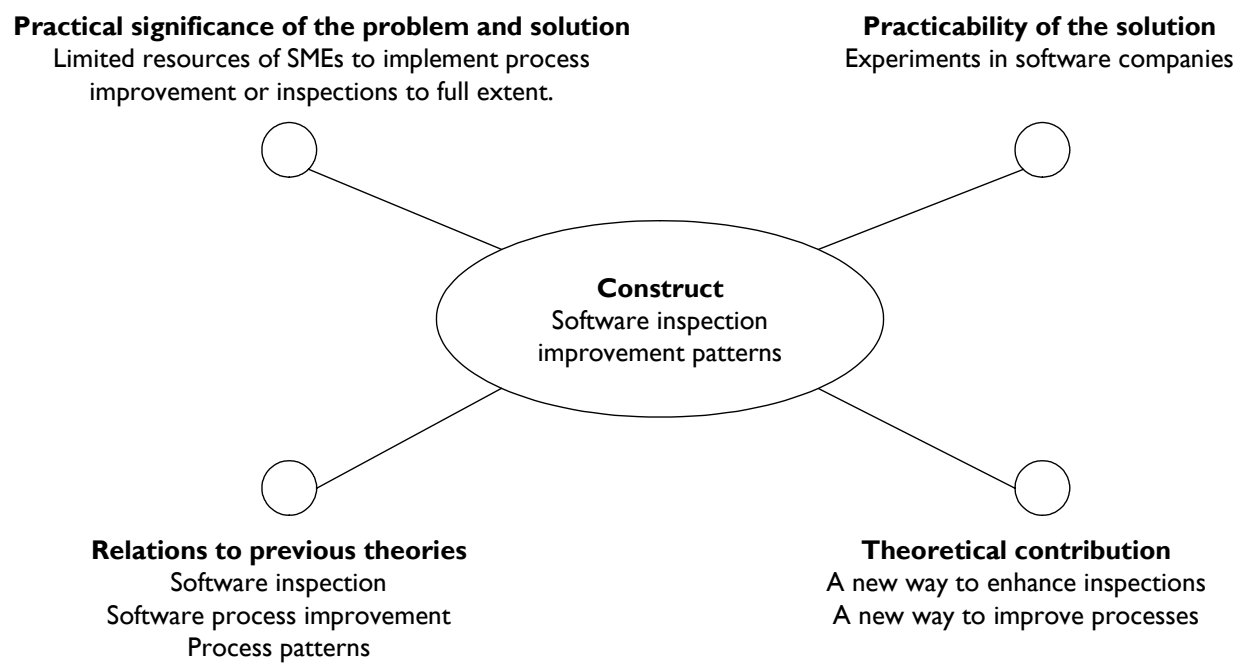

Fig. 1. This study in the context of constructive research, based on (Lukka 2003).

Merely building a system itself is not research. However, in software engineering, the role of systems is significant and investigating new technologies or ideas through systems development may contribute to the research. According to the principles of the discipline of engineering, a close cooperation between theory and practice is desirable in systems development methodology. Systems can be built to demonstrate its feasibility and measuring properties, to improve the performance of systems, to describe behaviours of systems, to improve the performance of systems and to review prior research. According to Nunamaker, no one research method is sufficient by itself in information systems research. Using a multi-methodological approach helps in gaining a more complete understanding of the subject of the research (Nunamaker et al. 1990).

One considerable dilemma in constructive research is the question over the scientist's ability to maintain an objective and critical attitude during the development and evaluation of the innovative construction (Lukka 2003). In this research, objective results are achieved by active experimentation in all phases of the research. Scientific relevance has been evaluated by publishing findings in scientific forums.

As a research process, constructive research typically consists of the following phases, according to Lukka (2003): 
- Find a practically-relevant problem

- Examine the potential for research co-operation

- Obtain a deep understanding of the topic area

- Innovate a solution idea and develop a problem-solving construction

- Implement and test the solution

- Consider the applicability of the solution

- Identify and analyse the theoretical contribution

In (Nunamaker et al. 1990), the following steps in systems development research are recognized:

- Construct a conceptual framework

- Develop a system architecture

- Analyse and design the system

- Build the system

- Experiment, observe and evaluate the system

The main structure of both of these research processes are similar and can be summarized in three key phases: theoretical analysis, construction and validation. These phases are depicted in Figure 2.

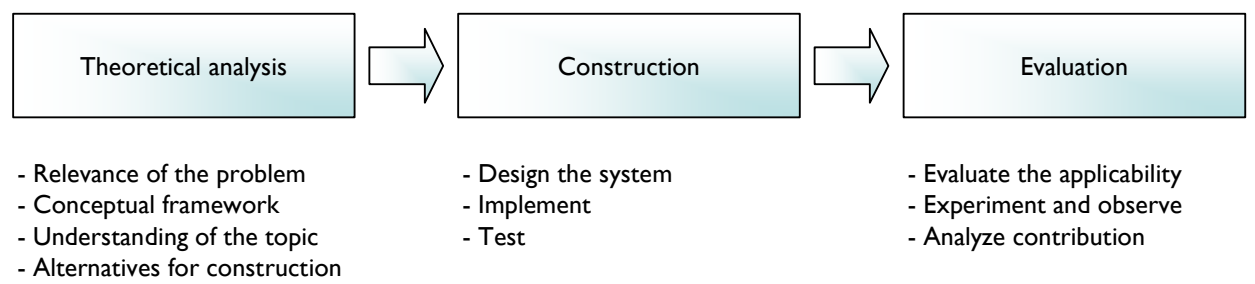

Fig. 2. Research process described in three stages.

In Figure 2, the steps defined in (Lukka 2003) and (Nunamaker et al. 1990) are combined into three stages. Before constructing a tool or model, its feasibility has to be analysed and its context has to be defined. Theories and previous research on the subject need to be identified and examined. The construction itself seldom contributes to the research - its academic value has to be validated through observation and experimentation. When the construction is the first of its kind, it proves that something can be done. Most often, however, constructions attempt to prove that something can be done better than before.

Hevner and his co-authors (Hevner et al. 2004) have derived seven guidelines for information systems research from design science principles. They emphasize the iterative nature of design science and stress the difference between design research and routine design or system building. The guidelines presented by these authors can be used to identify the scientific contribution that is needed to differentiate research from ordinary building of an artefact. The seven guidelines are represented in Table 1. The guidelines are also compared with this study. 
Table 1. Design science research guidelines in (Hevner et al. 2004) compared with this study.

\begin{tabular}{|c|c|}
\hline Guideline & Description \\
\hline 1. Design as an artefact & $\begin{array}{l}\text { A viable construct, model, method or instantiation must be produced. In } \\
\text { this study, the artefacts are the software inspection capability model and } \\
\text { the catalogue of process patterns for improving the inspection process. }\end{array}$ \\
\hline 2. Problem relevance & $\begin{array}{l}\text { An unsolved, important and relevant business problem must be addressed. } \\
\text { The relevance of the problem is demonstrated in the theoretical analysis } \\
\text { and interviews reported in paper VI. }\end{array}$ \\
\hline 3. Design evaluation & $\begin{array}{l}\text { The quality of the artefact must be demonstrated. In this study, the } \\
\text { capability model and patterns have been tested in the industrial } \\
\text { environment. }\end{array}$ \\
\hline 4. Research contributions & $\begin{array}{l}\text { Clear and verifiable contributions must be identified. This study provides } \\
\text { new ideas for defining and improving the software inspection process. }\end{array}$ \\
\hline 5. Research rigor & $\begin{array}{l}\text { Construction and evaluation of the design artefact must be carried out } \\
\text { scientifically. In this study, several approaches have been used to validate } \\
\text { the results, in different phases of the research. Each experiment has been } \\
\text { planned separately and carefully. }\end{array}$ \\
\hline 6. Design as a search process & $\begin{array}{l}\text { The artefact is created in an iterative process until it satisfies the problem } \\
\text { environment. During this study, feedback about the research artefacts is } \\
\text { gathered from real-life environments and the artefacts have been refined } \\
\text { according to the feedback. }\end{array}$ \\
\hline 7. Communication of research & $\begin{array}{l}\text { Both technology-oriented and management-oriented audiences must be } \\
\text { taken into account when reporting the results. This study aims at } \\
\text { reporting the rationales for academics in a scientific format, and } \\
\text { presenting the capability model and pattern catalogue in as practicable } \\
\text { ways as possible. }\end{array}$ \\
\hline
\end{tabular}

The research question represented in this thesis has been approached from several viewpoints. The first papers concentrate on finding flexible inspection methods that would be suitable for small and dynamic organizations. Inspection tools and processes that have lower level of rigor have been studied. These findings provide the means to create a comprehensive understanding of the most essential elements of the inspection process and further enable the creation of a model for inspection capability. The capability model is introduced in latter articles and the most recent papers introduce and evaluate the pattern approach, which is built on the results of all the previous work.

The background and position of each paper is listed in Table 2. The table hence shows the overall flow of the research. The left-hand column identifies the individual papers. For each paper, its general theme, data-collection methods and research interests are listed. The two first papers introduced flexible, tool supported inspection processes that were evaluated in separate student experiments. The third paper involves gathering of empirical data from five companies. The fourth and fifth paper provide a more detailed view on one case company. The sixth paper provides a combined synopsis of the separate empirical studies, and the seventh paper is based on literature review. 
Table 2. Papers introduced during this research.

\begin{tabular}{|c|c|c|c|}
\hline Paper & Theme & Research interests & Research approach \\
\hline $\begin{array}{l}\text { HICSS-98, } \\
\text { AI-02 }\end{array}$ & $\begin{array}{l}\text { Computer supported software } \\
\text { inspection and flexible forms } \\
\text { of inspection }\end{array}$ & $\begin{array}{l}\text { How can an organization } \\
\text { introduce an inspection } \\
\text { method with the aid of } \\
\text { software tools? }\end{array}$ & $\begin{array}{l}\text { Construction and student } \\
\text { experiments }\end{array}$ \\
\hline APAQS-01 & $\begin{array}{l}\text { A process model for software } \\
\text { inspection }\end{array}$ & $\begin{array}{l}\text { What should an inspection } \\
\text { process model look like? }\end{array}$ & Case studies \\
\hline $\begin{array}{l}\text { PROFES- } \\
04\end{array}$ & $\begin{array}{l}\text { Improvement of the inspection } \\
\text { process based on the process } \\
\text { model }\end{array}$ & $\begin{array}{l}\text { Can process patterns be } \\
\text { applied to the inspection } \\
\text { improvement? }\end{array}$ & $\begin{array}{l}\text { Literature research, case } \\
\text { studies }\end{array}$ \\
\hline QSIC-04 & $\begin{array}{l}\text { Testing the inspection process } \\
\text { improvement model }\end{array}$ & $\begin{array}{l}\text { How well does the } \\
\text { improvement pattern approach } \\
\text { work? }\end{array}$ & Action research \\
\hline PNSQ-04 & $\begin{array}{l}\text { Summarizing the aspects of } \\
\text { inspections in SMEs }\end{array}$ & $\begin{array}{l}\text { What are the motives for and } \\
\text { obstacles to using inspections? }\end{array}$ & Interviews \\
\hline SPIP-05 & $\begin{array}{l}\text { Introducing the improvement } \\
\text { pattern approach }\end{array}$ & $\begin{array}{l}\text { Do the inspection literature } \\
\text { and other research reports } \\
\text { support the pattern approach? }\end{array}$ & Literature research \\
\hline
\end{tabular}

Both industrial and student experiments have been carried out to validate the ideas represented in these papers. Empirical data has been gathered individually for each piece of work, as the articles deal with different aspects of the problem. Questionnaires have been used in student experiments to collect data; observations and interviews have been used in the industrial environment.

The main idea of the research has evolved during the course of the work from lightweight process models and tool support to a complete inspection process model and methodology for assessing and improving the effectiveness of the process.

\subsection{Scope and Contributions of the Research}

The areas covered in this thesis are depicted in the Venn diagram in Figure 3. The main background theories of this work concern software inspection, software process improvement and patterns. In addition, a viewpoint involving small and medium sized enterprises is taken. This is due to the industrial experiments carried out during the research. The research problem was prompted by the needs of small companies, and experiments concerning inspection tools, flexible inspection methods, inspection assessments and improvements have been conducted in small companies or independent departments within larger organizations. There is, however, no reason to believe that the results would not be applicable in larger companies as well. 


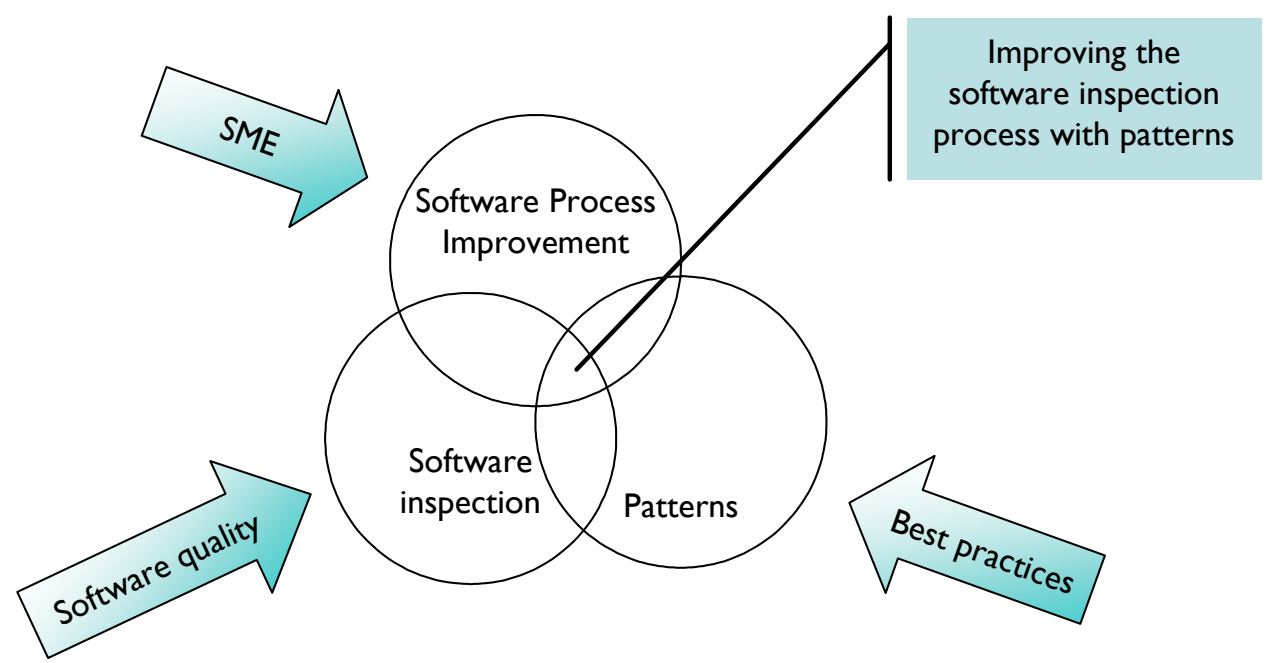

Fig. 3. Areas of interest in this research.

The main background theory for this research concerns software inspections. The essential elements of the process need to be identified, as well as the problems and benefits that have been reported when using inspections. Careful analysis of these elements, problems and benefits enables us to build an ideal model of the process. That, in turn, enables the evaluation of different types of inspection processes and the adjustment of the process for different purposes. This analysis itself serves researchers and practitioners working in the area of software inspections by helping to compare different types of inspections or combine best practices from the methods in order to find best inspection solutions to varying situations.

Ideas for inspection process improvement have to be taken from general software process improvement research and this is the second major topic of this research. The structures of the best known SPI methods are examined and factors that are reported to have an influence on SPI success are analysed. There are two concerns when studying the process improvement articles and research reports. Firstly, success factors and elements of SPI programmes have to be proportioned to small and medium sized organizations, because these organizations are especially addressed in this study. Secondly, little has been written about the actual and physical actions that have been taken to achieve successful improvements. Of course, specific procedures that are useful in one organization can be ineffective in another, and researchers have to summarize and generalize their experiences in the articles. Because this study focuses on software inspections, the concreteness of advisable improvement actions can be increased considerably.

The area of software patterns is the third major research topic. Patterns have been applied exceedingly successfully in software design and implementation. Due to this success, the approach has been experimented with in other areas of software engineering as well. This study examines the origins of patterns, different pattern formats, and the factors that constitute a practicable pattern. Not many research articles report on 
experiences of process pattern usage. Furthermore, the procedure of capturing software engineering knowledge in pattern form has not been especially well described in research. This would be important in enabling evaluation of the rationales behind patterns. In this study, attempts are made to describe in detail the evolution of the inspection process improvement pattern language, in order to contribute to software pattern research.

Even though the use of process patterns affects working practices in an organization, the enormous research field of organizational change is not the main focus of this thesis. Using the inspection process improvement model and pattern language presented in this work may also have organization-wide effects. This study, however, concentrates on the inspection process in SME companies, which are dynamic by nature. Managing and analysing larger social, political and other implications on the organizational level requires supplementary research efforts.

\subsection{Structure of the Thesis}

The rest of this work is arranged as follows.

In Chapter Two, the most widespread inspection methods are described. Special attention is given to the original Fagan inspection and the process introduced by Gilb and Graham. Alternative models are covered in less detail. However, ideas and elements that differ significantly from these two methods are described.

In Chapter Three, an overview of the colossal area of software process improvement is presented. Again, the most important models are represented. At the end of the chapter, requirements for the improvement model for inspection are outlined, given the special features concerning small companies and lessons learned from generic SPI models.

Chapter Four outlines the concepts of patterns and process patterns, and gives a brief introduction to the research carried out in this field. As almost anything can be presented as patterns, the presentation focuses mostly on patterns that have been validated. The best known pattern examples are Alexandrian patterns for architecture and design patterns. Examples of other pattern domains are also addressed.

Chapter Five introduces the inspection model constructed during this study. The foundation of the model was constructed based on inspection and process improvement literature. The elements of the model are explained and rationalized against previous knowledge in these areas.

Chapter Six introduces the pattern approach for inspection improvement. The structure and usage of patterns that have been extracted from literature or industrial experiments are described. Results from the experiments carried out to validate the patterns are also summarized. 


\section{Software Inspection Process}

Software inspection is a proven and widely known quality assurance method. In its traditional form it is a formal process involving labour-intensive manual analysis techniques. Software inspection involves careful examination of the code, design and other software artefacts, checking them for characteristics that are known to be problematic from past experience. Inspections are carried out at the beginning of the development cycle to identify and remove potential problems when fixing them is least expensive.

Software inspections are related to other peer review techniques, such as team reviews (Wiegers 2002) and walkthroughs (Humphrey 1989). The currently popular eXtreme Programming (Beck 2000) introduces the concept of pair programming, which can also be considered a peer review involving only two people. Wiegers (2002) categorizes three other techniques (peer deskcheck, passaround and ad hoc review) as peer reviews. However, software inspection is the most formal and systematic of these methods. It has also been addressed in research more than its counterparts.

Inspections are non-execution based, i.e., the inspector does not compile or execute the code during the examination. Thus, inspections can be applied to code documents long before tests are designed or even run. Humphrey (1989) states that in order to ensure a quality product, inspections should take place before the first compile or test. On the other hand, Gilb and Graham (1993), as well as Ebenau and Strauss (1993) consider that compiling the code is an entry criterion that has to be passed before an inspection. A compiler is a cheaper way to find syntax-related defects than the inspector.

Thus, software inspection is a process carried out manually by a group of developers on each other's code and other software artefacts in the early stages of the development cycle. Although the method requires human effort, it is very cost-effective, as defects can be removed before they enter the later implementation or maintenance phases.

\subsection{Inspection Process Models}

The software inspection method for reducing defects in source code and design documentation was first introduced by Michael Fagan in the middle of 1970's (Fagan 1976). The idea of inspection goes back even further - Weinberg's concept of egoless 
programming (Weinberg 1971) already introduces the idea of exposing one's source code for collegial technical review. In Fagan's article, however, the term software inspection was first introduced, and most importantly, some concrete results and benefits of the inspection process were presented.

The inspection literature describes several inspection methods. The most widespread and best known are the original Fagan process and Gilb's slightly enhanced process. These models are also the most successful, because they are the most precisely defined and documented. Many of the other methods and techniques are quite well-known, but not all of them have been studied carefully in order to validate their quality.

Software inspection and other reviewing methods have been subjects of active research during the last three decades (Wiegers 2002, Laitenberger et al. 2000). A number of researchers have made suggestions in order to improve the Fagan's original method. Structural modifications to the process, as well as techniques to support carrying out the process have been suggested in different studies. Although the inspection process has matured, there are still a number of open research questions regarding to carrying out the process as effectively as possible. (Aurum et al. 2002)

\subsubsection{Fagan Inspection}

The original inspection process was introduced almost 30 years ago by Michael Fagan. He has also published an update (Fagan 1986). The original article already states objectives beyond error detection for the process. According to Fagan (1976), inspections are a "formal, efficient, and economical method of finding errors in design and code". Fagan also defined an error as "any condition that causes a malfunction or that precludes the attainment of expected or previously specified results". Later, errors are referred to in the context of software inspections as defects. A deviation between a specification document and the corresponding code, for example, is a defect.

Figure 4 represents the phases in the Fagan code inspection method and their objectives. 


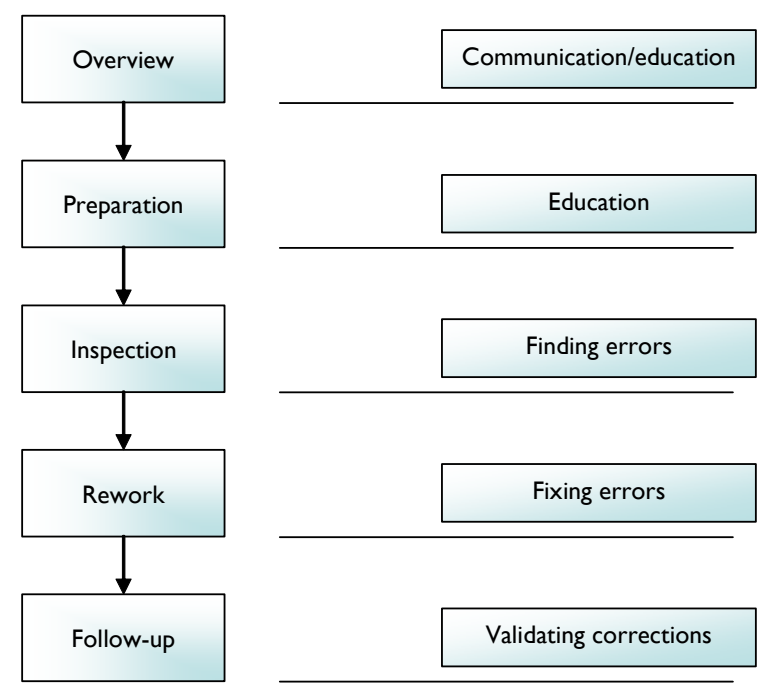

Fig. 4. The phases of the Fagan inspection process, and their corresponding objectives (Fagan 1976).

There are five stages in the process. Inspection starts with an overview that involves the entire inspection team. During the overview, the source code or design document under inspection is distributed to the team members and the author of the documentation explains his design and coding rationales. In the overview phase a common understanding of the artefact is gained and the overall objectives of the inspection are explained to the participants.

During the preparation phase, each team member individually reads through the document under inspection. Fagan emphasizes that the most important goal of this phase is to understand the artefact thoroughly, and only a few defects are found. In later inspection literature it has been argued - for example by Gilb and Graham (1993) - that, in fact, most defects are found already during the individual inspection. Arguments have been presented either way, but in the original Fagan method, defect detection in this phase is not an explicit goal.

After preparation, the actual error-finding phase, inspection, is performed. The whole team participates and the source code or design document under inspection is read through carefully. Solutions to problems are not discussed deeply and they are recorded only if they are obvious. Defects found are categorized as missing, wrong or extra, and their severity is determined as major or minor. The meeting should be quite fast-paced to be as effective as possible.

A written report of the findings ought to be produced within one day, to guide the rework and follow-up phases. The author of the inspected document then fixes the problems detected, in the rework phase. Finally, a follow-up phase occurs, where it is ensured that all required modifications have been made. It is also possible that the document will need either partial or complete re-inspection. 
Fagan already introduces the concepts of inspector roles and checklists to make the process efficient. He defines four roles: The moderator manages the inspection team, and he or she should be specially trained for that role. The other roles are designer, coder and tester, who are responsible for the software design, implementation and testing, respectively. The coder usually acts as a reader in the inspection meeting, presenting the implementation to the other inspectors.

Checklists can be used during the preparation and inspection phases to give clues as to what to look for in the document. Checklists can also provide the basis for defect-type classification - problems may concern logic, external interfaces or design, for example. Thus, the final categorization of defects includes error-type (from checklists), cause (missing, wrong or extra) and severity (major or minor).

Fagan also compares the inspection method to walkthroughs, especially to the one described in (Waldstein 1974). He stresses that inspection is more formal and brings significant benefits over walkthroughs in the form of error-finding efficiency, costeffectiveness, improved process scheduling and tracking and knowledge sharing. The utilization of checklists and roles is a notable improvement over walkthroughs. The formality and data-analysis aspects of inspections also provide better foundation for overall process improvement within the organization.

A strong emphasis is also placed on the training aspect. The preparation phase has been included in the process for educational purposes. Reporting and analysing the results is important in order to constantly improve the efficiency of error detection. Furthermore, Fagan emphasizes that proper training is required to carry out the inspection process correctly.

\subsubsection{Gilb and Graham Inspection}

Gilb and Graham's (1993) inspection process is based on the original Fagan method, but is even more precisely and comprehensively defined and described. In this method, process improvement is given more attention than in Fagan's original method. Formality of the process is ensured by emphasizing inspection planning activities and several templates for reporting and categorizing defects are provided. The phases of the GilbGraham inspection process and their objectives are illustrated in Figure 5. 


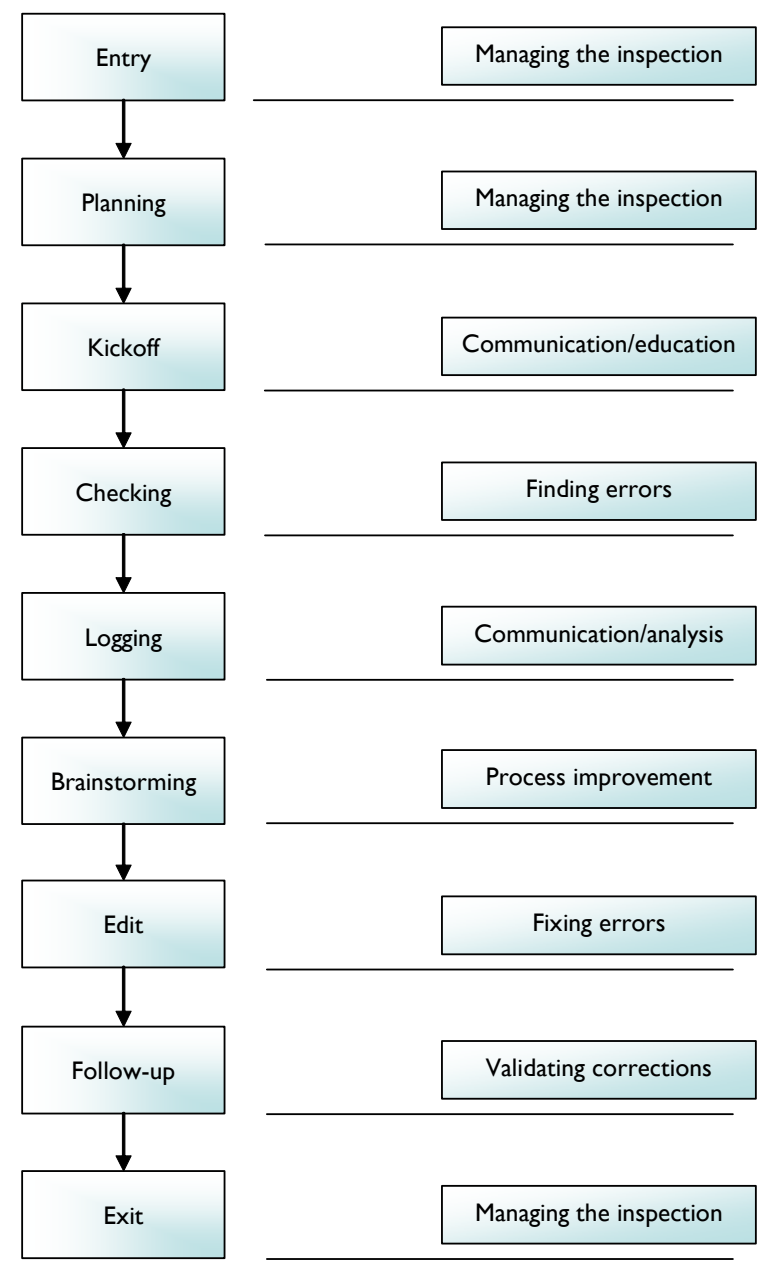

Fig. 5. The phases of the inspection process represented by Gilb and Graham, with corresponding objectives (Gilb \& Graham 1993).

The most important difference from Fagan's original inspection method concerns the checking and logging phases. In Gilb and Graham's method, most defect detection is performed during the individual checking, and the logging meeting is arranged to collect and initially categorize the findings. Some new findings may arise in the meeting, but the main objective is to reach agreement on the issues found individually.

The roles in the Gilb and Graham process are more inspection-related than in Fagan's method: the leader manages the inspection, the author is the producer of the document undergoing inspection and a checker is a person who tries to find the defects in the document. During the meeting one of the checkers acts as a scribe. Roles similar to those in Fagan's method (thus, roles which are more related to development aspects) can be used by the checkers as needed. 


\subsubsection{Wiegers Inspection}

The process presented by Karl Wiegers (2002) is shown in Figure 6. This inspection process consists of seven phases that are quite similar to the model presented by Gilb and Graham (1993).

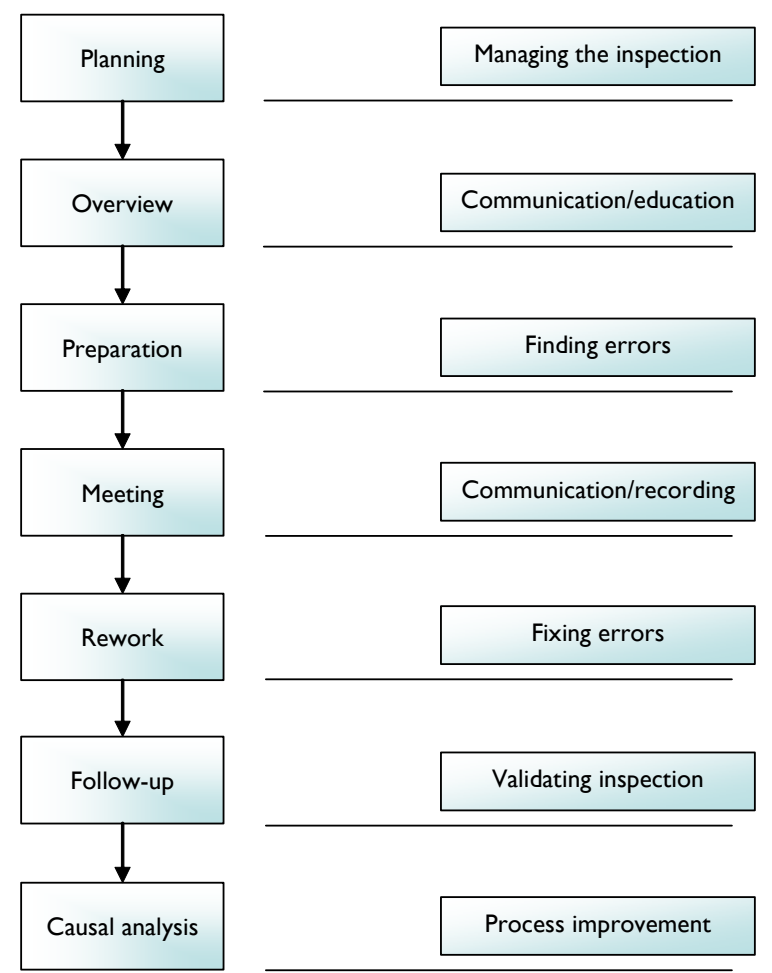

Fig. 6. The phases of Wiegers' inspection process, and their corresponding objectives (Wiegers 2002).

The author initiates the planning phase and also explains his or her objectives for the inspection to the moderator. Based on those objectives the moderator and the author together determine who should participate in the inspection and how the document should be split into parts for the inspection. These decisions are made based on historical inspection data collected within the organization. After the line-up and schedule has been decided, the moderator distributes the document, supporting documents, checklists and other materials to the participants.

The overview phase is typically arranged as an informal meeting. Inspection material can be distributed in the overview meeting. If the author can summarize the upcoming inspection and the document inspection in the material package or the participants are already adequately familiar with the material, this phase can be omitted.

The preparation and meeting phases are the essence of Wiegers' inspection process. He claims that dropping one of these stages results in a reduced number of discovered 
defects. According to Wiegers (2002), an inspection process without individual preparation should be called a walkthrough and an inspection without a meeting is a passaround.

During the preparation phase, the inspectors can use various tools and analysis methods to check that the document conforms to its specification and to the standards used in the project. Thus, the applicable rule sets, standards and specifications have to be available to the inspectors in the preparation phase, along with other checking aids, such as checklists.

In the meeting, a reader describes the document under inspection to the participants, a small portion at a time. After reading, the inspectors point out questions, comments and possible defects. A recorder notes the findings and an issue log is produced for the author. The moderator produces a summary report for the management, leaving out details of the issues found.

In the rework phase, the author addresses all the entries in the issue log, makes sure he or she understands each comment, and judges whether it is a defect. Defects are then corrected. Records for issues that have not caused modifications to the document are also saved in a defect tracking system for possible future actions.

The follow-up phase is necessary in order to bring the inspection to a close. In this phase, the author and moderator meet to ensure that all issues and defects have been resolved. The corrected document is also examined to verify that corrections have been made acceptably. After follow-up, the corrected document is baselined.

Finally, to maximize the benefits of the collected inspection data, a causal analysis phase is arranged. This corresponds to the brainstorming stage in Gilb and Graham's inspection process model. In this phase, the root causes of the defects are sought, in order to improve the development process. The performance of individual inspectors is not explored (Wiegers 2002).

\subsubsection{IEEE Standard for Software Reviews and Audits}

IEEE presented a standard for software review process in 1989. The standard describes the process flow for an effective review or audit. The standard defines a review as "an evaluation of software element(s) or project status to ascertain discrepancies from planned results and to recommend improvement." The evaluation is expected to follow a formal process. Software inspections and walkthroughs are given as examples of these processes (ANSI/IEEE 1989).

The standard defines the software inspection process as a rigorous peer examination consisting of six steps that are illustrated in Figure 7. The steps very closely follow Fagan's original code inspection method. 


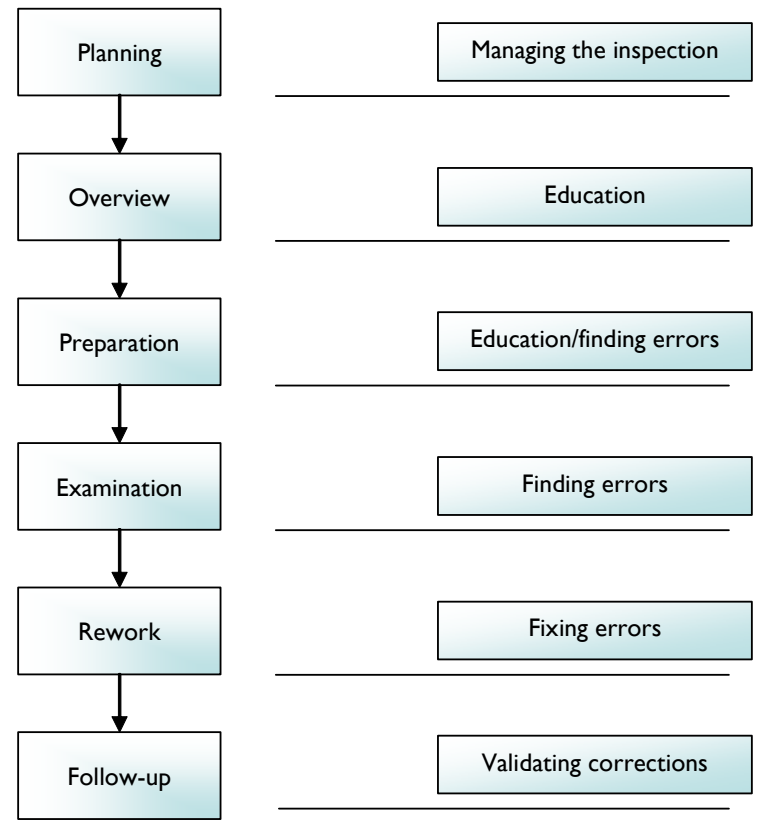

Fig. 7. The phases of the IEEE Standard inspection process, and their corresponding objectives (ANSI/IEEE 1989).

During the planning phase, the author and moderator together set up the schedule and material for the inspection iteration. The overview phase is also conducted by the author and moderator, and its purpose is to ensure that the members of the inspection team have adequate information about the software elements that are going to be inspected. During the preparation phase, inspectors are expected to familiarize themselves with the artefact under inspection, using checklists, specifications and other information that was presented in the overview.

During the examination phase, defects are identified and recorded. The examination is carried out in an inspection meeting, for which the standard provides a fixed agenda. During the meeting, the moderator reminds the inspectors about the procedural issues and ensures that preparation is done carefully by each inspector. The document under inspection is then read, and defects are recorded. Before closing the meeting, the defect list is reviewed and an unambiguous exit decision is made.

After examination, the errors discovered are fixed in the rework phase and the corrections are confirmed in the follow-up. The follow-up phase also involves reporting the inspection data (ANSI/IEEE 1989). 


\subsubsection{Alternative Processes}

Many variations of the inspection process exist. Alternative processes have been sought because inspections have been applied in heterogeneous environments and for very different types of artefacts. In particular, the high rigidity of the process has encouraged researchers to investigate more flexible forms of inspection. Inspection process flexibility means more independence of time and place, and, most importantly, tailorability. Some organizations wish to carry out inspections exhaustively, while others, especially small companies, desire light-weight but efficient inspections. Flexibility has been achieved by combining or removing selected phases in the process.

The effectiveness of inspections has also been a topic for active research. There has been a noticeable shift in the order of importance of the inspection phases. In the original Fagan model, the purpose of the preparation phase was to make the inspectors familiar with the artefact, and the actual defect-finding was done during the inspection meeting. More recently, it has been shown that individual preparation and reading can be at least as efficient as the traditional meeting in terms of revealing defects (Gilb \& Graham 1993, Votta 1993, Johnson 1994).

The alternative processes have altered such elements as the stages within the inspection process, the number of participants, the roles of inspectors and the supporting tools and materials for inspectors. The relationship between an inspector's individual work and joint meetings has been especially well targeted in research. Applying the method to different types of documentation has initiated discussion of the inspector roles, reading techniques and tools needed to enhance inspections. The integration of analytical techniques and measurement of the products and processes into the inspection process has also been studied, and promising results have been presented in that area, e.g., by Knight and Myers (1993).

The Active Design Review method was created to ensure complete coverage of design documents. The method suggests using several small and focused inspection meetings instead of one meeting involving a large number of people. In each of these smaller meetings, specific roles are assigned to the inspectors in order to reveal different types of defect. Reviewers and their roles are chosen based on the inspectors' skills, and each section of a document must undergo each type of review. The ADR process consists of three stages: overview, review and discussion. The discussion stage may involve several meetings, if there are unclear issues or disagreements between reviewers and developers. The stages of the ADR process are depicted in Figure 8 (Parnas \& Weiss 1985).

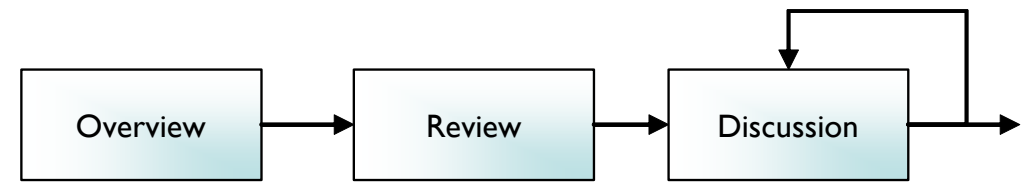

Fig. 8. The Active Design Review process (Parnas \& Weiss 1985). 
Schneider, Martin and Tsai (1992) have introduced N-Fold Inspections, in which many parallel inspections are performed by separate teams on the same artefact. The assumption is that a single inspection team will only find a fraction of the defects, and therefore multiple teams would not significantly duplicate each other's work. Figure 9 illustrates the phases in the N-Fold inspection process (Schneider et al. 1992).

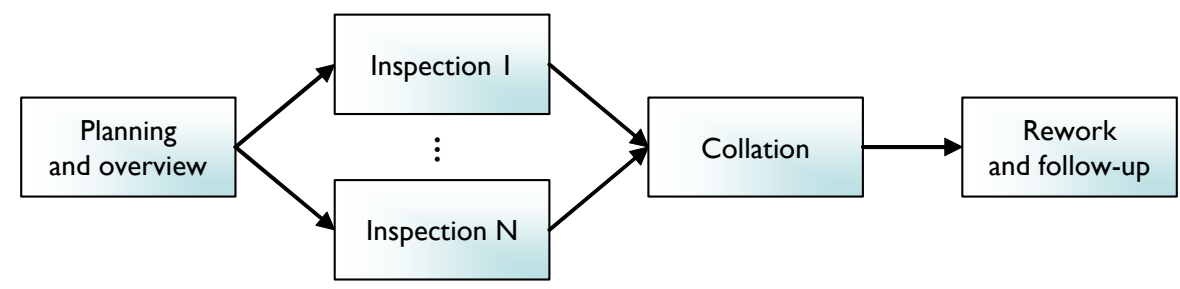

Fig. 9. The N-Fold inspection process (Schneider et al. 1992).

Sample-Driven Inspections is a method designed to reduce the effort required during an inspection session by focusing on the artefacts that are believed to contain the most defects. After deciding the sampling technique, defect-searching is divided into a preinspection part, during which samples of the artefacts are inspected to estimate which of them contain the most faults, and the main inspection, which is then carried out on the selected artefacts. Resourcing is carried out between the inspection phases. The quality and characteristics of the parts of the documentation determine which parts need closer attention. For example, safety-critical parts could be given higher priority than other parts of the artefact (Thelin et al. 2001). Figure 10 illustrates the sample-driven inspection process.

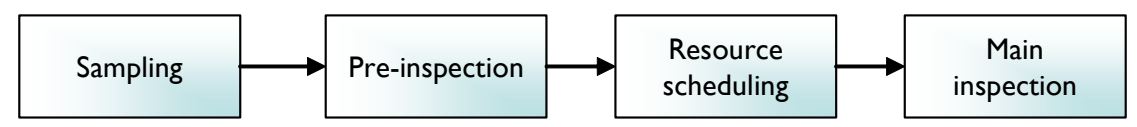

Fig. 10. The Sample-Driven inspection process (Thelin et al. 2001).

Knight and Myers (1991) suggest an enhanced inspection method which they call phased inspections. The objective of phased inspections is to provide a method that is tailorable, efficient in its resource consumption, rigorous and computer-supported. The work product is reviewed in a series of small inspections, called phases. Each phase ascertains that a certain characteristic of the document under inspection is correct. Each phase also assumes that a document that has passed the previous phase possesses the properties desired in that phase. In addition to error detection, phased inspections are intended to ensure that work products have certain quality characteristics, such as maintainability or portability. The phased inspection process is depicted in Figure 11. 
34

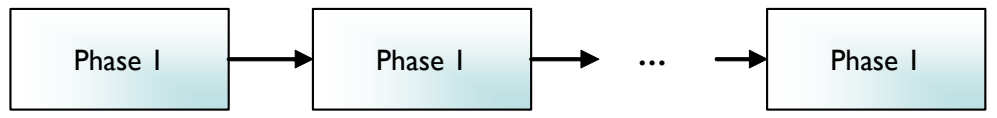

Fig. 11. The phased inspection process (Knight \& Myers 1991).

Phases are either single-inspector phases or multiple-inspector phases. A single-inspector phase is driven by a list of checks, and the document has to pass all the checks. In multiple-inspector phases the document is first read through by the participants individually. The inspectors then meet to compare their findings. The inspectors have reference documents and lists of checks to support them. Together, the phases form a single phased inspection, in which all important characteristics will be covered (Knight \& Myers 1993).

In a method called High-Impact inspection (SQE 2005, Wiegers 2002), various analysis techniques are used to discover defects. In addition to finding errors in the work product, this method is used to gain a deeper understanding of product quality. The method consists of briefing, examination and discussion stages, which correspond to the first three phases in Fagan inspections, or the kickoff, preparation and logging phases in the Gilb \& Graham model (Wiegers 2002).

Yourdon's Structured Walkthrough is a less formal and less rigorous method than Fagan's inspection. The aim is still similar. The process starts with a phase called organization, during which the author supplies the documentation and it is distributed to participants. A preparation phase follows. During the preparation phase the author should be available to answer questions concerning the material. During the walkthrough itself, the participants comment on the documentation. Comments that were made during the preparation can be handed directly to the author, if they do not need further clarification. After that, the author reworks the documentation. The final stage is follow-up, to ensure that the required changes are made to the reviewed document (Yourdon 1989). The process of structured walkthrough is identical to the original Fagan description, with two exceptions: the planning phase in Fagan's model is called organization, and Fagan's inspection phase is called walkthrough in the structured walkthrough model.

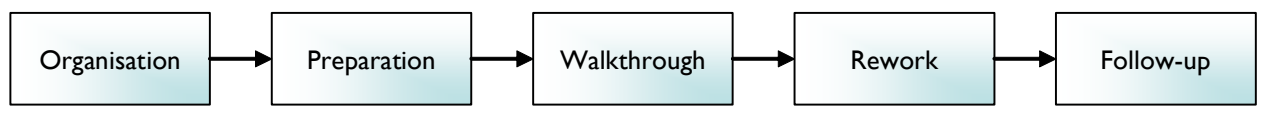

Fig. 12. The structured walkthrough process (Yourdon 1989).

Radice (2002) suggests a process model for inspection that consists of eight phases. The inspection starts with a planning and scheduling phase, followed by an optional overview phase, then preparation and inspection meeting phases, an optional analysis meeting phase, a rework phase, a follow up phase and finally a prevention meeting phase. In addition to these steps, the inspection is monitored and data is recorded and reported throughout the process. Even though overview and analysis meetings are not mandatory, 
they should not be omitted without an official decision (Radice 2002). Thus, the Radice model relies heavily on meetings.

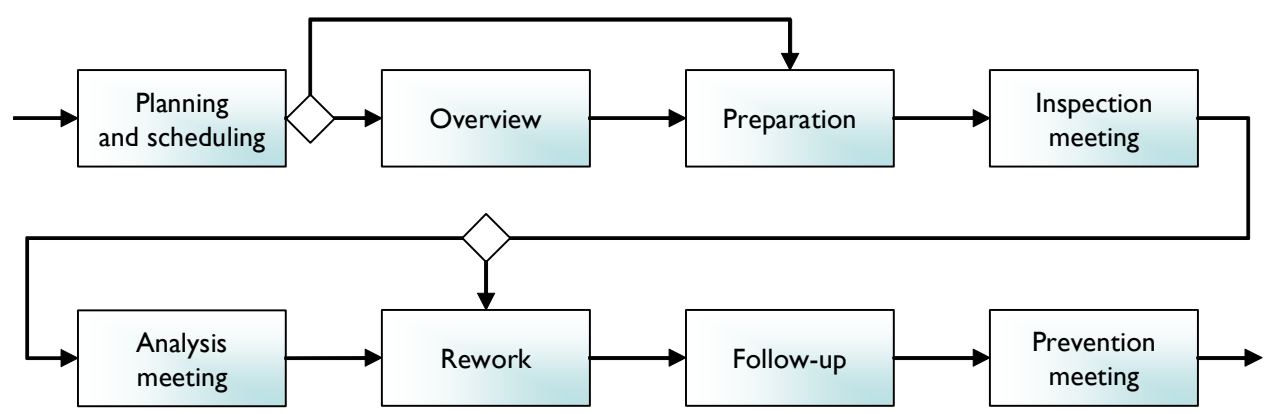

Fig. 13. Radice's inspection process (Radice 2002).

Even though there are a number of different inspection methods available, all of them have similarities. The basic idea has remained the same since the original Fagan method, but according to researchers' biases, additional characteristics have been integrated into the process. On the other hand, it has been suggested that certain parts - especially the meeting phase - could be removed from the process to save resources and time.

\subsection{Supporting Tools and Materials}

A number of tools and techniques have been introduced in order to make inspections even more efficient and to overcome some difficulties that have been encountered with the process. Two aspects in particular have been actively studied: 1) reading techniques that are intended for individual inspectors to help find defects more effectively and 2) software tools that either help to manage the inspection process and data related to it, or enable inspections to be carried out more effectively than with just a pen and paper.

Porter and Votta (1997) found that the inspection process variation used does not affect the defect detection results as much as the techniques and technology supporting individual inspectors during their reading tasks. Providing appropriate support to individual inspectors may increase their effectiveness significantly.

\subsubsection{Reading Techniques}

Recently, the focus for detecting defects in inspections has moved from the group meeting phase to the preparation phase. The reading technique used by the individual inspector has become one of the key elements of the inspection process. Reading techniques are used to help inspectors in their preparations and in finding defects effectively in the document under inspection. 
Reading the material under inspection without any guidance is called ad-hoc reading. It is obvious that inspecting without instructions requires an exceptionally proficient inspector. Reviewing the material can be done more efficiently - even by experienced inspectors - when a suitable set of guidelines is provided. Checklists are fundamental tools for this. A checklist includes questions to which the inspector finds answers from the documentation. The questions in the checklist are based on the design rules and standards used within the organization. Questions are typically answered "yes" or "no", and if "no" is given as an answer, a defect is found. Inspectors can have different checklists depending on the types of defects being looked for in the documentation and the type of artefact under inspection (Gilb \& Graham 1993).

The usage of checklists can be enhanced with roles. A checklist can include items that are important from a certain viewpoint, i.e., a specific specialist role can be assigned to each inspector. Organized by roles, checklists can be more detailed and more severe defects can be found in particular areas. Furthermore, the discovery of overlapping defects may be reduced by defining distinct roles. Figure 14 illustrates the usage of roles. In this example, maintainer, user and tester are the roles defined, and inspectors use these viewpoints when reading through the documentation. To ensure adequate coverage, a general checklist is required, with items that are not too specifically defined (the "overall" view in the figure).

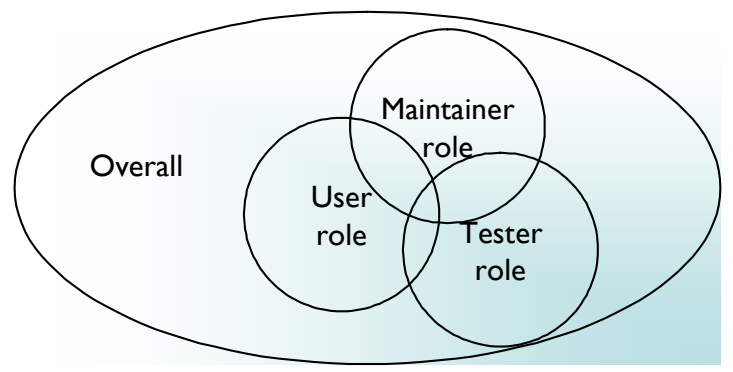

Fig. 14. Using roles makes defect-finding more efficient.

Checklist-based inspection is considered to be a basic reading technique. In addition, scenario-based techniques exist to ensure that the scope of the inspection is adequate (Basili et al. 1996). Reading techniques are substantial elements of inspections and they can have a bigger impact on inspection efficiency than any other feature of the process (Porter \& Votta 1997). Furthermore, the readability and clarity of documents improves over time, as authors become aware that artefacts will be inspected using reading techniques (Travassos et al. 1999).

Reading techniques can be constructive or analytical. Constructive techniques help in understanding the structure and operation of a system, while analytical techniques are used to evaluate the quality attributes of the artefact (Basili et al. 1996).

A step-wise abstraction reading technique provides a more structured reading method than ad-hoc reading or checklists. In step-wise abstraction, the inspectors start with the simplest code components, try to understand them and formulate higher-level abstractions 
that describe the functionality of the components. This process is iterated into even more higher levels of abstraction until the artefact is fully described and understood (Basili \& Mills 1982). The final description is then compared to the original specification, and differences between the two identify potential defects in the artefact.

A scenario describes how a certain piece of information can be found and what it should look like. Scenario-based reading techniques can offer a couple of significant advantages over checklist-based reading. A checklist helps with remembering certain aspects, but does not define possible locations of the defects. Scenarios attempt to define the locations as well. Checklists typically target the whole document under inspection, and the document may be read through several times during the review, while scenarios focus on a certain part of the document and are used to reveal certain types of defects. A further problem with checklists is their generality - questions in checklists are often too generic to be really useful (Tervonen 1996, Laitenberger \& DeBaud. 2000).

In scenario-based reading (Porter et al. 1995), each inspector is given a different scenario that includes questions and instructions on how to perform the reading. A scenario is defined as a "collection of procedures that operationalize strategies for detecting particular classes of defects". To obtain adequate coverage of the document under inspection, several scenarios (i.e., several inspectors) are needed. The selection and definition of scenarios is the most important success factor in scenario-based reading, and several approaches for creating scenarios have been proposed. Cheng and Jeffery (1996) suggest using function point analysis as a basis for the scenarios, and (Basili et al. 1996) introduces the concept of perspective-based reading, where the document is read from a certain stakeholder's viewpoint.

The perspective-based reading technique provides even more detailed reading instructions for inspectors. Inspectors reach an understanding of the document under inspection by constructing an appropriate abstraction as defined by the scenario (Laitenberger 2001). According to experiments reported in (Basili et al. 1996), less experienced inspectors in particular considered perspectives more helpful than checklists. Thelin, Runeson and Wohlin (2003) have introduced an usage-based reading technique that helps inspectors to focus on the most important issues. Their research also suggests that the usage-based reading technique is more efficient than traditional checklists.

Figure 15 summarizes the taxonomy of different reading techniques. 


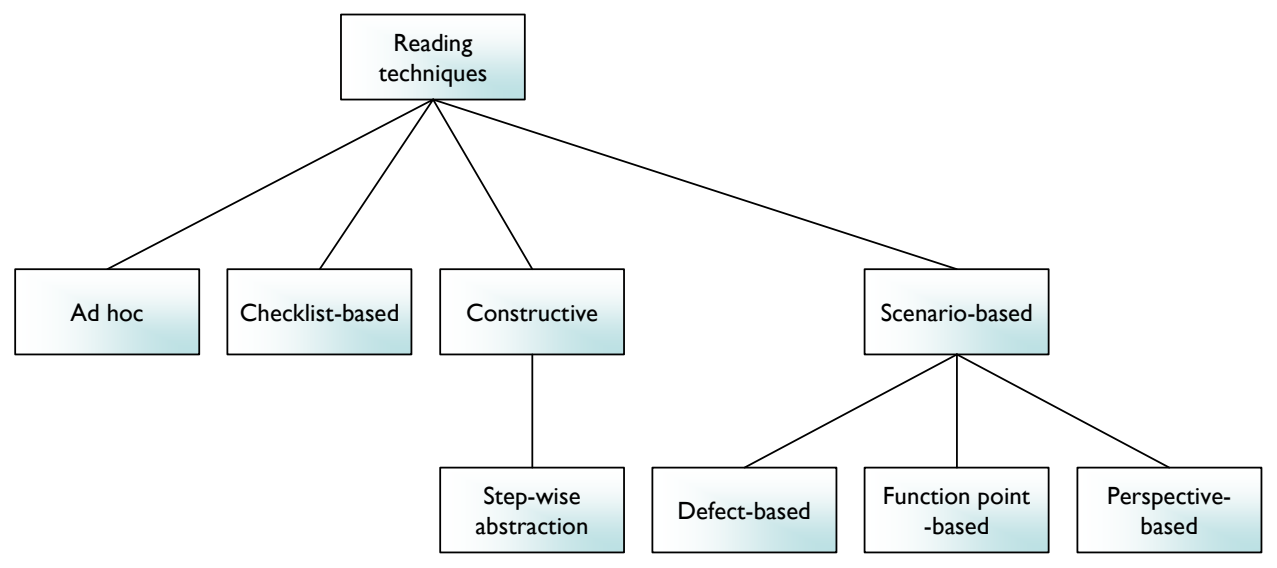

Fig. 15. Classes of reading techniques.

Ad hoc reading is performed without any specific instructions. Checklist-based reading is supported by checklists that may make use of different roles. Step-wise abstraction is a constructive reading technique, as it concentrates on understanding the functionality of the artefact. Defect-based scenarios, function point-based scenarios as suggested by Cheng and Jeffery (1996) and perspective-based reading are subcategories of scenariobased reading techniques.

\subsubsection{Computer-Supported Inspections}

Computer tools are an important part of an efficient software inspection process. Tools have been implemented for automatic defect detection, process management and reporting purposes, for example. There are situations in which tool support is essential to the efficient performance of inspections. If the development team is dispersed, tool support is necessary in order to carry out inspection tasks within a reasonable timeframe. Automating error-prone logging tasks makes meetings and statistical analysis considerably easier.

Tools can provide systems for electronic distribution of the inspection material, facilities for recording and attaching comments (suspected defects) directly on the document, classification of defects according to their severity and category, checklist management and reporting of inspection efficiency.

If development teams are geographically scattered and time limits are strict, the best solution is to arrange inspections virtually, through a network. Meeting-less inspections can be more cost-effective than traditional inspections (Porter et al. 1995, Votta 1993), but this usually requires a special infrastructure. The first inspection tools were aimed at supporting manual inspections, mainly providing help in error-prone logging tasks. The fundamental idea was the enabling of on-line issue recording. One of the first inspection tools providing that functionality was ICICLE (Brothers et al. 1990). The next crucial step forward was taken in Scrutiny (Gintell et al. 1993) and CSI (Mashaeykhi et al. 
1993), which made distributed inspections possible. The CAIS tool then brought in asynchronous inspection meetings (Mashayekhi et al. 1994). Experiments with these tools offered very promising results for time and place-independent inspection.

The World Wide Web provides a powerful infrastructure for collaborative inspection tools, featuring prevalence, platform independence and familiarity (Mahon 1996, Harjumaa \& Tervonen 2000). Since the HTML document format is supported by all of the most popular word processors, and web browsers can be enhanced to handle a variety of document types, virtually all written material becomes available for inspection. AISA (Stein et al. 1997) pioneered reviews of more expressive document types than plain text. In addition to AISA, WWW-based inspections were demonstrated with WiP (Harjumaa \& Tervonen 2000) and XATI (Hedberg 2004), for example.

Johnson and Tjahjono's (1998) CSRS tool and Macdonald's (1998) ASSIST tool enable the customization of the underlying inspection process for the needs of a particular project. Such tailorability is necessary for efficient support for distribution in different organizations and situations.

In addition, tools should not require extra work, but should integrate into the existing infrastructure and be able to handle all types of documentation. The XATI tool is the first inspection tool implementation that utilizes external data repositories for the management of inspection-related data and documents (Hedberg 2004). It is also possible to use offthe-shelf software products for inspection collaboration. For example, Microsoft Word includes features that enable distributed reviewing of documents, to some extent. Most software engineering documents are created with word processors, thus it would be very convenient to use the same software for both authoring and inspecting. Adobe Acrobat also includes features for online team collaboration, information sharing and document annotation (Harjumaa 2003). Figure 16 presents a screenshot showing a design document being commented with Acrobat software. 


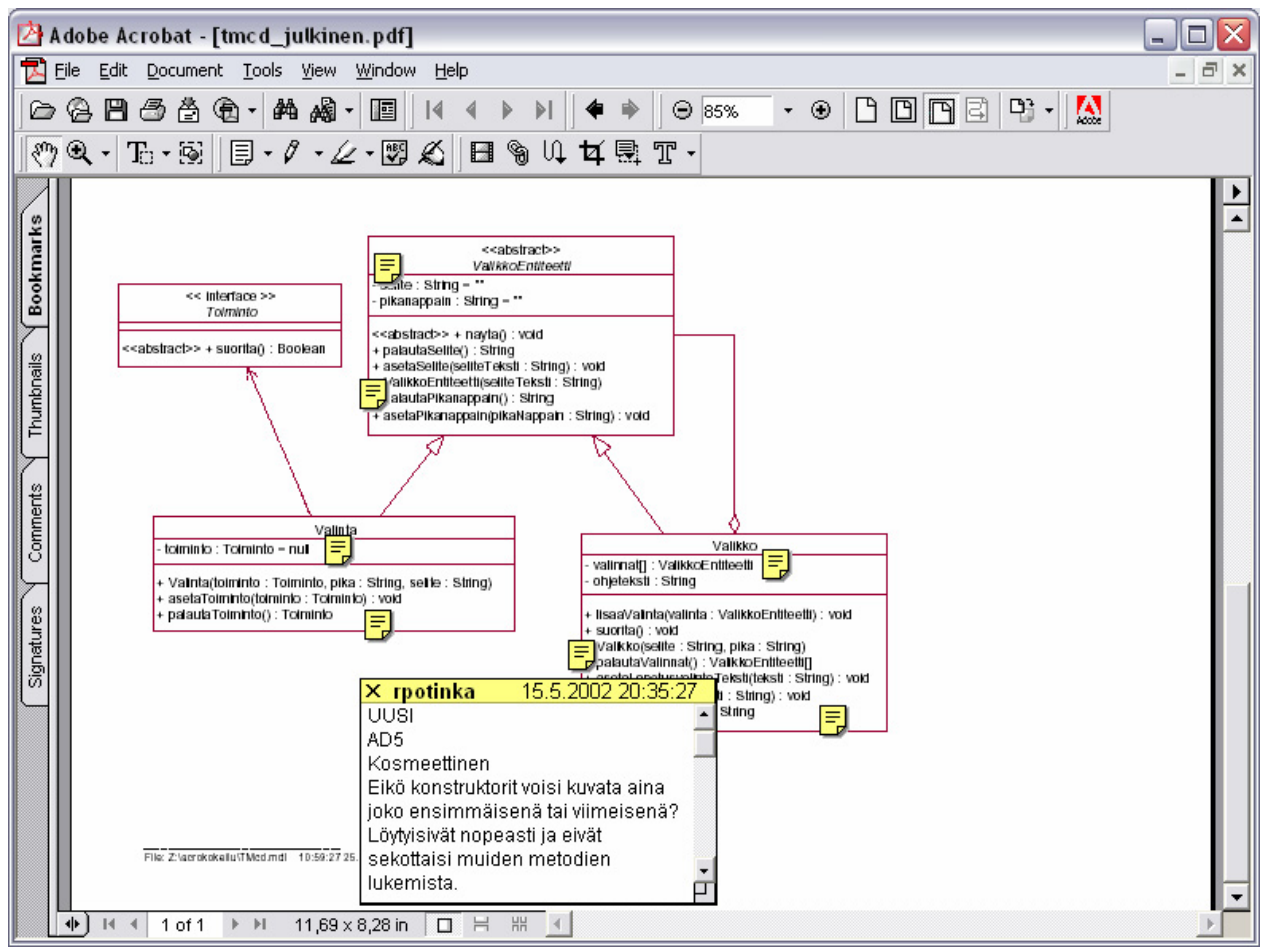

Fig. 16. A design document commented with the Adobe Acrobat tool (Harjumaa 2003).

There are also inspection tools that have been implemented to make defect detection more effective. The ICICLE tool (Brothers et al. 1990) already included functionality to automatically detect the most typical types of defects. Knight and Myers (1993) introduced a tool called InspeQ that provided support for automated defect detection in the Phased inspection process. CodeSurfer is a tool that is meant to provide inspectors with detailed analysis of the code that is being inspected. The tool shows the structure and dependencies of the source code visually, as graphs. Such static analysis can help the inspectors to focus on more challenging issues during the inspection (Anderson et al. 2003, Anderson \& Teitelbaum 2001).

Although the purpose of inspection tools is to provide assistance, they have to some extent reshaped and modernized the whole process. Today, it is not usual or acceptable to carry out inspections without appropriate tools for data entry, analysis and reporting purposes. The selection of a tool depends closely on the underlying infrastructure within the organization. Making use of existing data repositories, authoring tools, version management systems, collaborative systems and project management tools could significantly promote the employment of inspection tools. Integration would ensure the easy initiation and running of the inspection process. Interfaces to external systems, however, are extremely challenging to implement, as the variety of tools and procedures is enormous. Additionally, to provide support in every phase of the software development 
cycle, the tool would have to be capable of handling a variety of document types - such as design diagrams and hyperlinked documents.

It is reasonable to count computer-supported software inspection as a method of its own, separate from traditional software inspection. This computerized process could be called virtual software inspection. Virtual software inspection conforms to a defined workflow (or process) and is performed in a distributed manner with the aid of an inspection tool. The process can be lightweight or all-inclusive, and can include asynchronous and synchronous phases. It is usually carried out through a network, but traditional meetings can be included if necessary. Geographical distribution is typically achieved through the World Wide Web. An inspection tool is a software package particularly designed for inspection collaboration, and it should be capable of at least managing and delivering the inspection documentation on-line, enabling the effortless recording of defects and the automatic gathering of metrics.

Each inspection tool has its strengths. It seems that developers have had their own aspirations and ideas regarding computer-supported inspection, so that each tool implementation stresses some specific aspect of the software inspection method.

\subsection{Benefits}

Incorporating inspections into the software engineering process is not free. They can increase the total project budget between 5 and 15 percent (Gilb \& Graham 1993), and the decision as to whether to introduce inspections and what type of inspection process should be used has to be made on a case by case basis. There is a great deal of evidence that in most cases the results yielded by a well-defined inspection process outweigh the cost of performing them.

The first and obvious benefit of software inspections is the effect on product quality. It is not meaningful to directly compare different inspection methods or organizations by reported inspection results, as circumstances and objectives vary between the environments. Table 3 gives some examples of the effectiveness of inspection defectdetection, as reported in the literature.

Table 3. Software inspection success.

\begin{tabular}{lc}
\hline Source & Defect detection effectiveness \\
\hline (Fagan 1976) & $82 \%$ \\
(Fagan 1986) & $50-93 \%$ \\
Kitchenham et al. 1986) & $58 \%$ \\
(Collofello \& Woodfield 1989) & $54-64 \%$ \\
(Weller 1992) & $70 \%$ \\
(Shirey 1992) & $60-70 \%$ \\
(Grady \& van Slack 1994) & $60-70 \%$ \\
(Barnard \& Price 1994) & $30-75 \%$ \\
\hline
\end{tabular}


Fagan's original paper on inspections reports that 38 out of 46 defects were discovered by design and code inspections. Fagan's publication about projects in the Standard Bank of South Africa and IBM Respond in the UK reports that inspections found between 50 percent and 93 percent of all defects over the lifecycle of the products (Fagan 1976, Fagan 1986). In (Kitchenham et al. 1986), data from ICL shows that about $58 \%$ of defects can be found in inspections. Collofello and Woodfield (1989) have studied large real-time software development projects and report 54\% defect-detection coverage for design documents and even higher - 64\% - for code. Bull NH Information Systems also have positive experience of code inspections, which are reported to reveal $70 \%$ of all defects (Weller 1992, Weller 1993). Shirey (1992) states that typical defect-detection rates range from 60 to 70 percent. The same numbers are confirmed by Grady and van Slack (1994) in research carried out in a division in Hewlett-Packard. Barnard and Price (1994) note that several references estimate effectiveness as $30-75 \%$, and their own inspection experiment at AT\&T Bell Laboratories is reported to reveal more than $70 \%$ of all defects.

High defect-detection effectiveness produces better quality products that, in turn, reduce the total cost of development. When defects are prevented from shifting from the early development stages to the coding and maintenance stages, direct and considerable cost benefits are gained:

- Development productivity improves

- Development time is reduced

- Time required for testing is reduced

- Testing costs are reduced

- Maintenance costs are reduced.

Cutting down on the need to rework documentation always improves productivity. Inspections also motivate and educate the developers, further enhancing their productivity. The delivery times of software products are shortened, as designs and solutions can be evaluated during the early stages. The time required for the integration and system test/debug phases is also reduced. This directly reduces the effort and cost needed to test a product. Finally, a good quality product leads to lower lifetime costs and fewer fixes than a product that has not been systematically inspected.

Grady and van Slack (1994) report total savings of 33\% in the specification, design, code and test planning phases in Hewlett-Packard. Russell (1991) reports that inspections can be as much as 20 times more efficient than testing in revealing defects in large-scale development. Ebenau and Strauss (1993) give an example of a NASA software supplier company that is estimated to have saved 7.5 million dollars over 300 inspections. Thus, exact numbers explaining cost-savings in different sources vary, as development processes, people and tools are different in different organizations. However, it is clear that if defects are detected and corrected earlier, significant savings in development resources will be gained in the long run. According to Rico (2002), software inspections provide the highest "return of investment" factor amongst the most common methods.

The inspection process also brings some indirect advantages. Even though Fagan's original article concerned code inspections in particular, one can take advantage of inspections in phases prior to coding. Any human-readable artefact produced during the 
software development lifecycle can be inspected: requirement specifications, design documents and models, test plans and system documentation are all good candidates for review. In fact, inspections are one of the few quality assurance techniques available for artefacts other than code.

The importance of inspections in process improvement is unquestionable, and statistical data gathered during inspections is useful in causal analysis, defect tracking and when predicting the costs and work needed to produce software. In an organization functioning at a high level of software process maturity, the data collected from inspections can be used to improve the process further. Data from inspection summary reports can be used to identify the most common or most costly kinds of bugs, determine their root causes, and change how work is performed so as to prevent those types of errors (Gilb \& Graham 1993).

Improved quality, reduced delivery times and data for process improvement are significant managerial advantages of inspections, serving the whole company. On a smaller scale, individual developers can also gain considerably. It is their work products that inspections improve. Their work will be more valued and their own work will be easier as the products are more maintainable. Furthermore, inspections help the developers to keep themselves up-to-date concerning each other's work. Inspection meetings can greatly enrich communication and engineering knowledge within the organization (Gilb \& Graham 1993). This in turn helps in resource backup or replacement, building a team spirit and knowledge-sharing between the members of a development team.

Even though several researchers state that inspections are more efficient than testing, the method is not intended to replace testing. Inspections tend to reveal different kinds of errors than testing activities do. Comparing inspections to testing, Tian (2001) states that inspections are good for detecting static and localized faults, and also hidden faults that may not cause failures in the current environment and cannot be found by testing. Furthermore, inspection can continue after detecting a fault whereas testing may get stopped when a failure occurs (Tian 2001).

Table 4. Typical errors revealed by inspections and testing. (Tian 2001)

\begin{tabular}{lcc}
\hline Error type or location & Inspection & Testing \\
\hline Errors in module interfaces & $\mathrm{x}$ & \\
Complex code & $\mathrm{x}$ & \\
Unnecessary functionality & $\mathrm{x}$ & $\mathrm{x}$ \\
Poor usability & $\mathrm{x}$ & $\mathrm{x}$ \\
Poor performance & $\mathrm{x}$ & $\mathrm{x}$ \\
Bad code structure & $\mathrm{x}$ & $\mathrm{x}$ \\
Failure to meet requirements & $\mathrm{x}$ & \\
Boundary value errors & & \\
\hline
\end{tabular}

Testing and inspection are not mutually exclusive. Instead they complement each other as quality assurance techniques, both improving different aspects of product quality. Fagan (1976) reports that inspection pays off even if the same code later goes through testing. 


\subsection{Problems}

The inspection process is not completely unproblematic. For example, Ciolkovski, Laitenberger and Biffl (2003) have found that reviews are still not practiced systematically, even though the method is well-known and dates back over 30 years. According to their research, there are lacks in several aspects. Reviews are not arranged in all relevant development phases. Furthermore, not all the steps of the review process itself are implemented. Process evaluation and improvement are seldom properly addressed in reviews.

Radice (2002) lists several people issues that may cause problems in inspections: a recorder records too slowly, defects are interpreted or recorded incorrectly, inspectors are not prepared for inspections, the desired people do not participate, people come late to meetings, the author is over-defensive, the author does not participate, the author makes repairs at the meetings or the author is unprepared.

In distributed software engineering particularly, meetings can be difficult to arrange. Settling a time and place that would fit all participants is challenging. Even though tools have been created for asynchronous and distributed inspections, distribution remains a challenge. Inspections also involve a number of error-prone or laborious tasks that require comprehensive tool support (Votta 1993, Johnson \& Tjahjono 1998).

Several textbooks concerning inspections emphasize that introducing the method may be difficult, mainly due to the investments required, and to developers' opposition. Authors may fear that their performance will be evaluated in inspections and people or teams will be benchmarked against each other. Thus, the establishment of the process has to be carried out carefully (Gilb \& Graham 1993, Wiegers 2002).

There can also be problems with the document type under inspection. For example, object-oriented software may be more difficult to understand than its procedural counterpart. Inheritance, polymorphism and use of design patterns may result in quite complex software structures. In addition, the run-time structure of an object-oriented program is very different from its compile-time structure. These aspects make inspection of object-oriented programs challenging (Dunsmore et al. 2002).

\subsection{Inspections in Small Companies}

There are a couple of issues related to software inspections that need special attention in small and medium sized organizations. First of all, adopting an inspection process can take years. Grady and van Slack (1994) report that in Hewlett-Packard, early experiments in 1976 did not develop into organization-wide inspection practices until the 1990s.

Both Fagan's and Gilb's inspection methods, which are the best known variations, are completely manual. This means that a lot of effort is required to fully implement these process models. In a small company, there can simply be too few people available to cover all the roles defined by the inspection. Using specialist roles and separate moderators may be difficult if only a few people are assigned to a development project. There will probably not be a distinct quality assurance group to manage inspections, train moderators, provide tools and templates and update checklists, for example. There is no 
point in running full-scale inspections and the process needs to be adapted and lightened. On the other hand, specific guidelines as to how the adaptation should be performed are not provided in detail in the inspection literature.

The most laborious parts of the process can be automated or supported by software tools. However, flexibility and interoperability aspects are absent in most current inspection tool implementations. This means that off-the-shelf tools cannot be easily tailored for different types of inspection processes. Furthermore, small companies seldom have the time or money to implement customized tools to meet their specific requirements. Compromises are necessary to relieve individual inspectors' efforts without considerable investments.

Even the actual format of the inspection process may seem unclear to those who are just starting inspections. In research, partially contradictory arguments have been used to describe the optimal inspection process. For example, it has been reported that few defects are discovered in inspection meetings; however, it has also been reported that groupware-enhanced meetings are very efficient (Porter et al. 1995).

Radice (2002) states that small teams would benefit from inspections even if the inspections are not practiced as formally as in larger organizations. Data-gathering and inspection efficiency improvement are still possible. Gilb and Graham (1993) also encourage small companies to adopt inspections. Overheads can be avoided by sampling the documents to be inspected and concentrating on the most essential metrics, for example.

\subsection{Summary}

Software inspection is a quality assurance process that encompasses several phases, such as planning, preparation, meeting, rework and follow-up. The method was originally introduced by Michael Fagan almost thirty years ago. Since then, numerous variations have been presented by researchers, but the essence of the process remains unchanged: a software artefact is carefully read through by peers, and dubious design solutions are communicated to the author.

Even though inspection was initially used for code, the technique is now applied to a wide range of document types including requirements and design documentation (Basili et al. 1996, Porter et al. 1995, Travassos et al. 1999). In addition to the expanding scope of the documentation, the concept of software inspection is continuously developing through the introduction of tools and supporting material for the application of the process.

A great deal of research has been reported in the area of software inspection since Fagan's original article in 1976. Many variations on the traditional inspection process described by Fagan have been proposed. Tools have been introduced to help inspectors find more defects and coordinate their work. Tools for automatic defect-detection have also been experimented with. Other defect detection aids, such as reading techniques, have been defined for different types of artefacts.

Although each variation has made alterations to the inspection process or modified the phases of the process, the fundamental elements of preparation, inspection, and 
rework/follow-up from Fagan's original description have remained present in later process models.

Software inspection is amongst the most effective methods when evaluating the return on investment of different quality assurance techniques. Inspections are useful from several viewpoints: in addition to clean-up and prevention of defects, they serve as a training method and valuable data source for knowledge-capturing and management (Biffl \& Halling 2003b, Rico 2002). The inspection process even provides means for process measurement and management, and can enhance team collaboration and communication (Gilb \& Graham 1993, Rico 2002).

Elements related to the inspection process are summarized in Figure 17. The actual process of reviewing an artefact is supported by computer tools and reading techniques that provide guidance, forms, templates, checklists, scenarios, reporting tools and other assistance, that help inspectors to carry out reviewing tasks as efficiently as possible and provide inspection leaders with efficient means for managing and coordinating the process.

The documentation under inspection is not the only target of improvement. Inspections produce a great deal of data that is useful in analysing and improving the whole development process. Of course, the same data can be used to measure the effectiveness of the inspection process itself, and to update checklists, decide optimal checking rates and numbers of participants, etc.

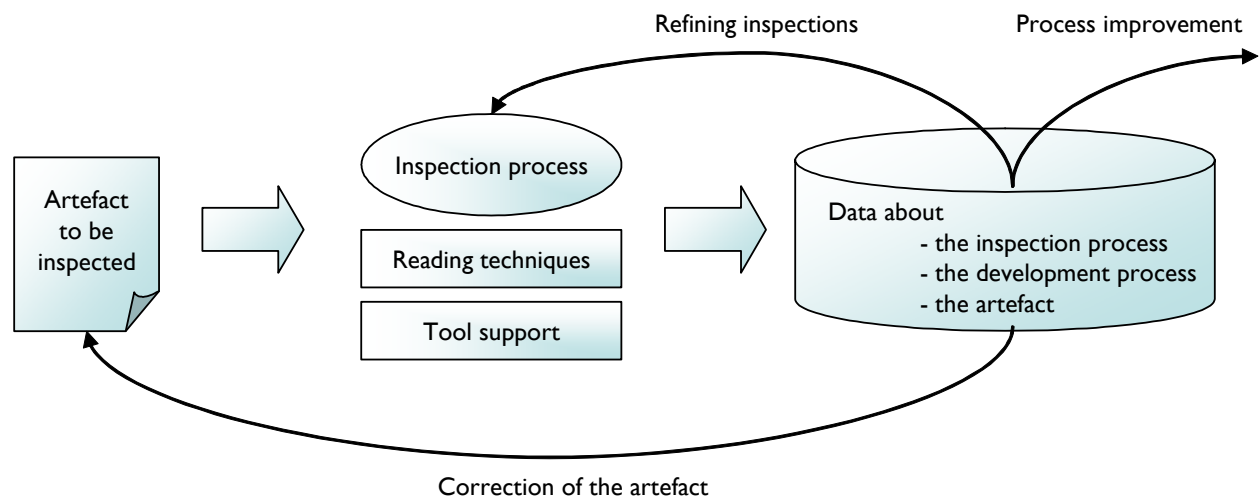

Fig. 17. The inspection process and its surroundings.

While calculations on inspection efficiency are typically quite rough and sometimes very optimistic, they clearly indicate that the software inspection process provides a considerable return on investment. Particularly in small companies, software inspection is a valuable quality assurance method.

The overall goals of the software inspection process can be summarized at the highest level as in Table 5. The first and most important benefit of inspections is the effectiveness of revealing defects and preventing them from proceeding to the following development phases. The number of defects also explains the overall quality of the product. Removal of the defects directly improves product quality, and further analysis of the data can be performed, to help understand and improve the entire development process. Reading 
through the software documentation in inspections also improves team communication and enables the transfer of good design solutions from experts to novices.

Table 5. Summary of high-level inspection goals.

\begin{tabular}{ll}
\hline Goal & Description \\
\hline Identifying defects & Identify defects in a document and track their origins. \\
Product estimation & Evaluate the quality of an artefact. \\
Improvement & Improve the quality of an artefact. \\
Process improvement & Provide data for improving the development process. \\
Communication & Improve the communication of a team. \\
Education & Provide the means for knowledge transfer. \\
\hline
\end{tabular}

Various methods, with different names, have emerged since 1976. Each technique has specifications for team size, roles for people and reports to make, but essentially, all methods concentrate on looking at the work product and discussing it. Depending on the approach, peer reviews are called code inspections, software inspections, structured walkthroughs, formal technical reviews, and so on. Despite the differences in name, the basic workflow is quite similar between the methods. 


\section{Software Process Improvement}

A software process defines the way in which different activities of software development and maintenance are organized, managed and measured. A definition given by Davenport (1993) states that a process is a structured, measured set of activities designed to produce a specific output for a particular customer or market.

The software development business has been subject to dramatic changes during the last couple of decades. The systems to be built are more complex than ever, including aspects of distribution, interoperability, and mobile platforms, and having very high usability demands. Development work has also changed, as companies have become global. Building such a complicated software product requires effort from a large number of specialists, perhaps working in different countries and continents. Without a defined workflow and regime to guide and organize their co-operation, meeting the quality demands of the project and the product would be impossible.

It is necessary for there to be an appropriate process infrastructure before management and improvement activities can be carried out effectively (Humphrey 1989). A defined process provides the company with a number of important things:

- It enables the analysis and improvement of the process.

- It improves predictability.

- It helps in understanding roles and dependencies.

- It provides a smooth working framework and guides decision-making. (Clough 1992)

Software processes can be represented at various levels of abstraction and exactness. Models can be generic life-cycle models, or organization- or project-specific. In addition to abstraction, models can embody several distinct viewpoints: functional, behavioural or organizational, for example. Classic examples of software processes include the waterfall model (Royce 1970) and the spiral model (Boehm 1988). The Rational Unified Process (Kruchten 2000) and eXtreme Programming (Beck 2000) are examples of more recent development process models. The selection of a development process model depends on the development environment - the organization, the people and the application domain.

Regardless of the process, current thinking holds that the quality of a software product is significantly affected by the quality of the process that produces it. The more systematic and manageable the process is, the higher the quality of the outcome (Deming 1982, Paulk 1995). 
Thus, it is possible to improve the outcomes of software development by improving the underlying process (Galin 2004, Basili \& Caldiera 1995), but there are also other viewpoints concerning process improvement. The more effective the process is, the shorter delivery times become and the lower the costs of producing the software (Herbsleb et al. 1994). The company's development maturity may be benchmarked, which may also initiate process improvement (Humphrey \& Sweet 1987).

Assessing the quality of software and assuring that it will not fail is difficult, as there are many aspects to quality. Furthermore, some quality attributes may be more important than others in different situations. For example, Ishikawa (1985) stresses customer satisfaction when defining quality, while McConnell $(1993,2002)$ measures quality with a set of -ilities (reliability, maintainability, usability, portability, etc.)

Assessing the quality of the software process may be even more complex. There are many human, cultural, domain-specific and other factors that cannot be completely modelled and managed systematically. The need to improve processes and remain competitive has been widely recognized, however, and software companies have invested a lot of resources in process improvement.

According to Grady (1997), software processes should be continuously improved and successful practices should be disseminated to different audiences to achieve their widespread usage. Improvements are achieved by modelling previous progress, creating similar situations and making people transfer as quickly as possible to the improved situations (Grady 1997, Basili et al. 1994).

\subsection{Assessment, Improvement and Innovation}

It is important to distinguish between the concepts of process assessment, improvement and innovation. Process assessment is a method for the examination and evaluation of an organization's development process without the intention to alter it. As seen in Figure 18, process improvement is a result of a quality program that continuously and incrementally changes the process, upgrading its performance. Process innovation is a more radical change, which involves the replacement of the entire process, or parts of it. (Hammer 1996) 


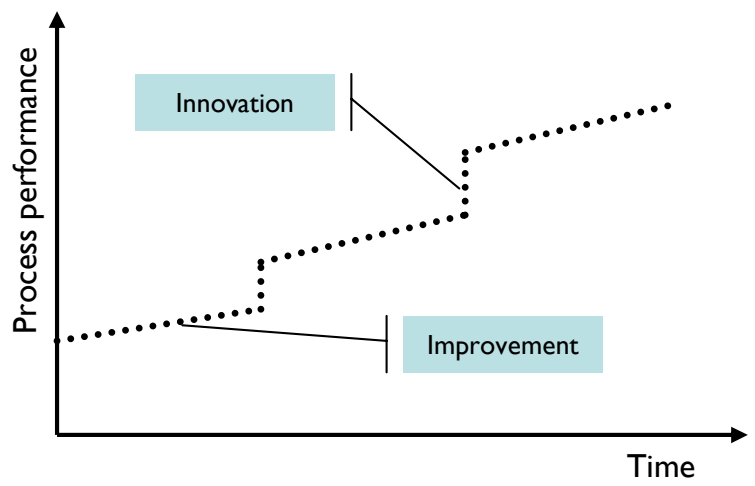

Fig. 18. Process improvement and process innovation. (Hammer 1996)

An assessment can be made against other organizations to benchmark companies or divisions and to gain an outside view on the process. For example, Heires (2001) provides an example of benchmarking. This type of comparison also enables the diffusion of best practices and ideas between organizations. Another possibility is to carry out the benchmarking against a reference model, such as CMM or Bootstrap. These assessment models provide an ideal list of best practices, to which the organization's practices are compared. There are a vast number of articles reporting model-based process improvement successes. The CMU/SEI-94-TR-13 report, published in 1994, provides an analysis and summary of CMM-based process improvement from 13 organizations (SEI 1994). Currently, model-based assessment is considered the most fundamental process assessment method. Finally, the assessment can be performed on the basis of the specific needs of the organization. The aim is to provide an effortless assessment approach, which is relevant and tailored to the needs of the company (Dybå \& Moe 1999).

The role of software process assessment is illustrated in Figure 19. The assessment is carried out by analysing all relevant data that can be measured and extracted from the production process. The challenge of this analysis is the validity of the data, as measuring a process may involve methodological and statistical problems (Bollinger \& McGowan 1991). In model-based assessment, the analysis is performed by comparing existing procedures, standards and work products to those defined in the reference model.

Assessment then enables both the determination of process capability, and the improvement of the process. Analysing the current status of the process motivates changes, which need to be defined by a process improvement programme. Improvement actions can also be determined using the reference model that has been chosen for the improvement. 


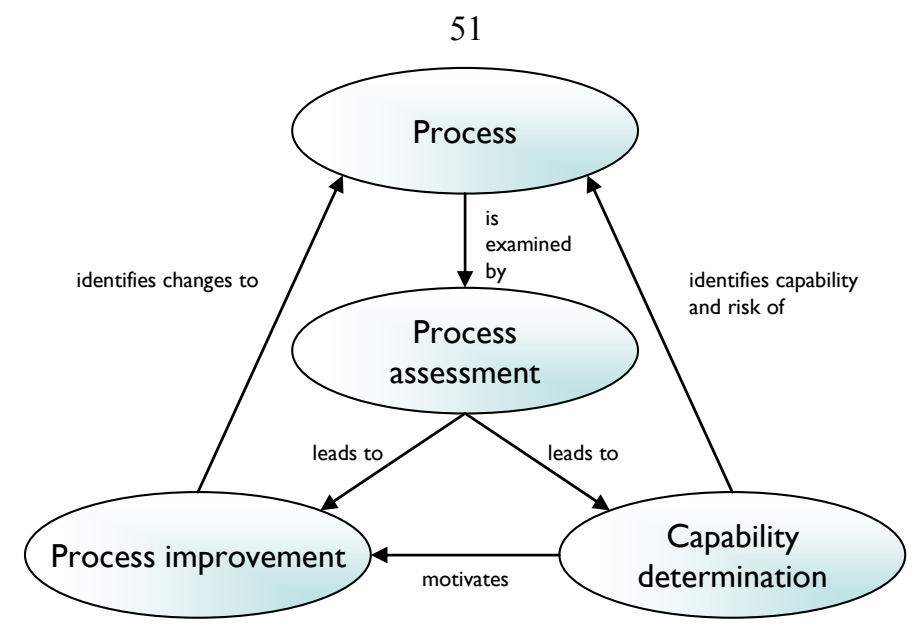

Fig. 19. The role of software process assessment. (ISO/IEC TR 15504-1:1998)

It has been generally accepted that SPI requires knowledge of the underlying process, meaning that before carrying out any improvement actions, the current status has to be assessed. A large body of literature exists concerning software process assessment: Humphrey (1989) and Zahran (1998), for example.

Assessment is just the first step in improvement. Furthermore, any change to a process will require time for its implementation and maturing before it becomes effective. Changes made at different times may increase productivity and quality slowly, over a long period of time. Finally, the benefit to the organization can take different forms: the same software can be produced with fewer resources, or more software requirements can be met for the same cost. Thus, understanding and managing all the aspects of SPI is challenging.

Improvements are typically trialled in one project before wider adoption. If the improvement appears successful, other teams and projects are encouraged to apply the same procedures. As the use of the new practices spreads, the documentation and measurement of the process becomes more precise. Thus, process improvement is an evolutionary process (Grady 1997).

\subsection{Models and Approaches}

A vast number of improvement methods exist. Many of them are unproven (Grady 1997). Each model describes different levels of process maturity, and provides instruments for systematic assessment of the organization's current operation. Use of SPI models may result in dramatic improvements in software quality by reducing the costs and time required for development, and increasing productivity (Butler 1995, Pitterman 2000, Yamamura 1999, Diaz \& Sligo 1997).

The fundamental philosophy for systematic and continuous improvement is provided by the Plan-Do-Check-Act (PDCA) model (Shewhart 1931). The PDCA model was 
developed by Walter Shewhart in the 1920s, and was introduced by W. E. Deming in his studies concerning Japanese post-war industry. The four phases of the model are repeated in spiral form, thus making the improvement systematic and on-going. The cycle is depicted in Figure 20. Even though the foundation of the model is in manufacturing, the PDCA approach provides a suitable foundation for almost any software process improvement model: it describes improvement actions at a high level, builds improvement on previous successes and encourages measurement of process quality.

The Plan-Do-Check-Act model includes four phases:

- Plan. The planning phase relies largely on software process assessments. During this phase, a focus for improvement actions is set. When designing the improvement, three aspects need to be considered: 1) Intended improvements have to serve the company's core business competencies. 2) The improvements have to be worth investment. The improvements have to be evaluated and alternative approaches have to be presented. 3) The organization has to be committed to the change.

- Do. The improvements planned are tried out in the "do" phase. During this phase, any obstacles or restraints have to be resolved and removed, and the people within the organization have to be convinced about the improvement. Adequate training and consultation are important for successful adaptation of new practices. Achievements have to be advertised to create a positive atmosphere for further improvements.

- Check. The final results of the improvements are evaluated during the "check" phase. The evaluations have to demonstrate practical benefits from a business point of view. Even though individual improvements may succeed without measurable goals, it is necessary to measure the success to accomplish organization-wide motivation and conviction.

- Act. The "act" phase emphasizes organizational decisions to enable further development of the processes. Management involvement, and reviews and revisions of successful process improvements are the key elements when converting new procedures into best practices and standards.

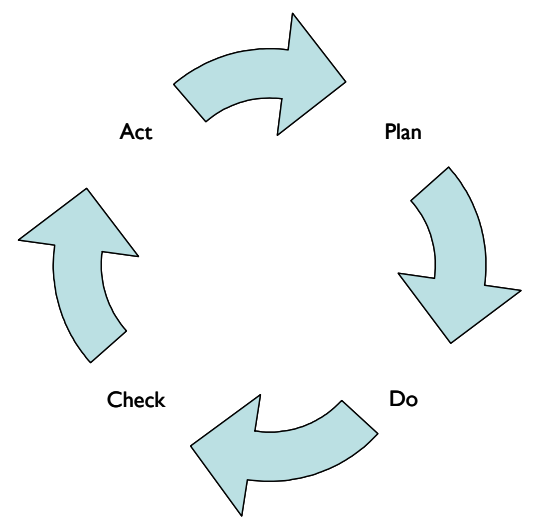

Fig. 20. The Shewhart (PDCA) improvement cycle. (Grady 1997) 
Shewhart's influence on quality management in Japanese companies was significant. His pioneering ideas and PDCA model led to increased productivity and market shares and his argument that reduced process variability would result in improved quality was well verified.

In the 1980s, W. Edwards Deming published the book "Out of the Crisis", in which he proposed a 14-step programme to transform an organization into a quality- and customeroriented organization. In his programme, he addresses the need for training, leadership and the role of each individual developer, but the most important points that he introduces are:

- Constancy of purpose

- Quality built into the product

- Culture of continuous improvement

"Constancy of purpose" means that a company has to invest resources in research and development to deal with problems and to find solutions that work in the long term. Deming also argued that it is necessary to improve the production process to build quality into the product instead of assigning resources for defect removal. Continuous improvement is a fundamental part of Deming's programme. An organization should be a learning organization, and all employees, processes and areas of development should be constantly improved (Deming 1982).

Other pioneering work in the area of process improvement has been carried out by Juran (1951), who introduced the Juran Trilogy of quality planning, control and improvement. Finally, Philip Crosby's Zero Defects programme outlines a philosophy of doing things right the first time. Crosby's Quality Management Maturity Grid measures the maturity of the organization's quality system. Different aspects of quality management are evaluated and rated on a scale of one to five (Crosby 1979). This grid has been later adapted into the Capability Maturity Model (CMM).

The Quality Improvement Paradigm (QIP) is based on the principles of the Deming's cycle and includes three major phases to achieve improvements: planning, execution and evaluation. QIP is developed in the context of software engineering and introduces a concept of experience packaging in order to enable organizational learning. Improvement actions in QIP are divided into project and organizational levels (Basili et al. 1994, Basili \& Caldiera 1995).

The project level cycle is concerned with the feedback that is gained during an execution of a project. The project cycle also provides data for the organization level cycle, enabling comparison of project performance to existing project data. Furthermore, reusable software assets can be identified and generalized for future use (Basili \& Caldiera 1995). The steps in QIP are repeated for continuous improvement.

\subsubsection{Capability Maturity Model for Software}

The Capability Maturity Model for Software was first introduced in 1987. It has gone through a number of revisions after that. The SW-CMM is a framework that describes the 
key elements of effective software product development to help the organization set goals for scheduling, cost, functionality and quality (Paulk et al. 1993).

The SW-CMM can be used for software process improvement, in which an organization plans and implements changes to its software processes. In addition, the SW-CMM can be used as a framework for software process assessments, in which software professionals determine the status of an organization's current software process, and for software capability evaluations, in which contractors who are qualified to perform the software work are identified or the state of the software process used in an existing development infrastructure is monitored.

According to the SW-CMM, a mature software process is well-defined, managed, controlled and effective. It should also have potential for growth and be consistent throughout the organization. The SW-CMM defines five incremental maturity levels for measuring the maturity of the software process within an organization. Each level focuses on one important process component. Achieving a higher maturity level results in an increase in the capability of the software process. The maturity levels of the SW-CMM are listed in Table 6.

Table 6. The maturity levels of the SW-CMM (Paulk et al. 1993).

\begin{tabular}{|c|c|}
\hline Level & Description \\
\hline 1 - Initial & $\begin{array}{l}\text { Process is ad-hoc or chaotic. Success depends on individuals and few process activities are } \\
\text { defined. }\end{array}$ \\
\hline 2 - Repeatable & $\begin{array}{l}\text { Cost-, schedule- and functionality-tracking by basic project management processes. Earlier } \\
\text { successes are repeated by initial process discipline. }\end{array}$ \\
\hline 3 - Defined & $\begin{array}{l}\text { A documented, standardized and integrated software process for both management and } \\
\text { engineering activities. Projects use processes that are tailored for the organization's } \\
\text { standard process. }\end{array}$ \\
\hline 4 - Managed & $\begin{array}{l}\text { Detailed measures of the process and product quality are collected. Quantitative } \\
\text { understanding and controlling of process and products. }\end{array}$ \\
\hline 5 - Optimizing & $\begin{array}{l}\text { Continuous process improvement by quantitative feedback and piloting of new ideas and } \\
\text { technologies. }\end{array}$ \\
\hline
\end{tabular}

Each maturity level consists of several key process areas that an organization must cover in order to achieve that maturity level. The key process areas of each maturity level provide capabilities that lead to improvement in the organization at that level. The first level is an exception: there are no key process areas defined for it. Maturity levels and their corresponding key process areas are listed below:

1. At the initial level, the software process of an organization is not stable and management practices are poor. The process is "ad hoc" and changes as work progresses, thus making it unpredictable. This level has no attached key process areas.

2. At the repeatable level, the objective is to establish basic project management controls that allow the organization to "repeat" successful practices used in earlier projects. Key process areas for this level are: management of requirements, software project planning, software project tracking and oversight, software subcontract management, software quality assurance, and software configuration management. 
3. At the defined level, both project and organizational aspects are taken into consideration. The activities are stable and repeatable and are implemented throughout the organization. Key process areas for this level are: organization process focus, organization process definition, training programs, integrated software management, software product engineering, inter-group coordination, and peer reviews.

4. At the managed level, the focus is on productivity and quality, and their assessment. Measurements are established for the quantitative assessment and evaluation of both software processes and the products being built. The objective is to predict quality trends and identify and correct causes of variation. Key process areas for this level are: quantitative process management and software quality management.

5. At the optimizing level, the organization continuously improves its software processes and measures improvements. The organization has the ability to identify process weaknesses and product defects, and prevent them from recurring. In addition, new technologies are brought into the processes in an orderly manner. Key process areas for this level are: defect-prevention, technology change management and process change management.

The SW-CMM is an incremental model, which means that to reach a certain maturity level, an organization has to implement all the practices described in that level and all preceding levels.

CMM can be applied in different manners. Satpathy and Harrison (2002) have introduced product-focused process improvement, for example. The idea is to recognize a limited set of quality factors related to the end-product, and implement only those improvement actions that can lead to improvement of the desired quality factor (Satpathy \& Harrison 2002).

As an example, Haley (1996) reports a successful SPI effort, in which CMM was used to make the software process more predictable and effective over the course of eight years. This report concerns a large company that can establish an effective infrastructure for continuous improvement, and has the resources for large-scale measurement and analysis of project data. Diaz and Sligo (1997) report experiences from a division of Motorola and state that a shift from one maturity level to a higher level reduces the defect density by a factor of two. Galin (2004) summarizes four cases of CMM implementation demonstrating significant increases in productivity and customer satisfaction, as the defect densities in software products reduced dramatically.

The SW-CMM is the oldest, best known and most commonly-applied assessment method. Still, there are also risks in CMM-based improvement. Lack of management support, inability to learn from previous experiences, concentrating too much on technical issues and resistance against change are the challenges most often mentioned (Grady 1997, McFeeley 1996).

\subsubsection{CMMI}

Since their introduction in the early 1990s, Capability Maturity Models (CMMs) have been developed for a wide range of disciplines. The Software Engineering Institute (SEI) has created separate models for systems engineering (SE), software engineering (SW), 
software acquisition, workforce management and development, and integrated process and product development (IPPD). Other organizations have also created models based on the CMM, adding to the excess of models. Even though these models have been valuable to many organizations, the use of several models has been complicated. Companies that attempt process improvement across disciplines have had to cope with different model architectures and approaches. Focusing the improvement strategy can be difficult when training, assessments and procedures for the improvement vary across models.

From 1998, software CMM development efforts were directed towards the Capability Maturity Model Integration (CMMI), to overcome these problems. The Capability Maturity Model Integration (CMMI) is an effort to create a single model integrating existing disciplines into one framework and providing an expandable framework for the introduction of new disciplines as needs arise. The CMMI product suite contains a number of models that embody different bodies of knowledge. The development of software systems is addressed by the CMMI-SW model.

For an easy migration from CMM to CMMI, two versions of the CMMI framework were introduced:

1. The staged version

2. The continuous version.

The selection between these two depends on the specific needs of the organization. The staged version is more familiar to organizations that have been using the CMM, and the continuous version allows the organization to focus on the most important business objectives. Furthermore, the continuous version is ISO15504 compliant (Galin 2004).

Version 1.1 of the CMMI integrates the disciplines of software engineering, systems engineering, integrated product and process development, and supplier sourcing into one process improvement framework. The CMMI does not intend to provide techniques to be used in the different process areas, as the number of addressed areas is vast and taking all possible situations into consideration would be impossible. However, some of the process areas include recommended techniques presented as sidebars. Furthermore, the model can be extended with new disciplines as needed.

The CMMI-SW model focuses on software engineering. Its goal is to apply a systematic and quantifiable approach to the development, operation and maintenance of software systems. Software engineering best practices that were already covered by the SW-CMM, such as configuration management, project management and peer reviews, are now included in the CMMI-SW model (Ahern et al. 2001).

As a process improvement model, CMMI shows what to do, not how to do it or who should do it. To actually perform the practices outlined by the model, practicable processes and techniques have to be established to achieve the improvement.

\subsubsection{Bootstrap}

Bootstrap is a European counterpart of SW-CMM. The initial version of the method was developed in the ESPRIT project (Kuvaja et al. 1994). The approach is based on CMM, ISO9000 series and the Software Engineering Standards of the European Space Agency 
(ESA). The Bootstrap method widens the assessment scope to the organizational level. Furthermore, assessment is more fine-grained than in CMM: the five capability levels are refined into quarters. Figure 21 illustrates the Bootstrap capability levels. Using the quarter-divisions helps managers and assessors to identify how far the organization is from the next level. This also motivates improvement.

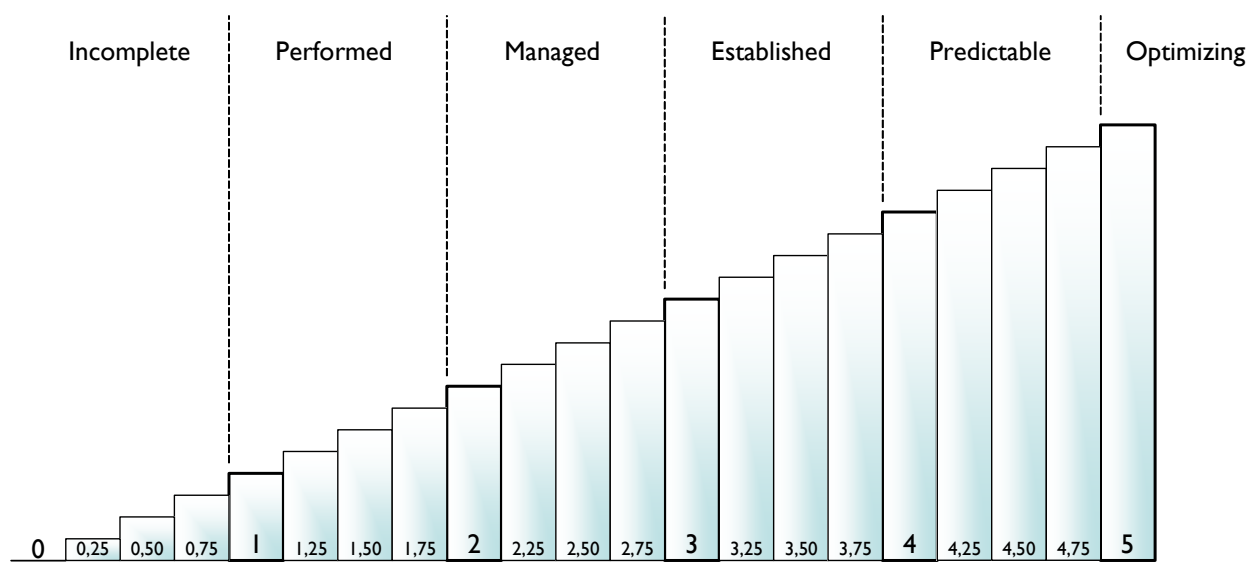

Fig. 21. Bootstrap capability levels. (Kuvaja et al. 1994)

The main advantage of the Bootstrap model is that it is ISO 15504 compliant, but it also allows the selection of the processes to be assessed. This makes it easier to evaluate only a portion of the development organization, and to target improvement at the most critical operations. Managing and understanding the improvement initiative is also more straightforward.

Bootstrap dissociates process and capability dimensions. The underlying philosophy is that the improvement should be driven by organizational needs. The process dimension provides a framework for improving the individual processes (Kuvaja et al. 1994). Figure 22 illustrates the process dimension of the Bootstrap model. Each process is assessed based on its goals, scope and purpose. Processes are composed of base practices, which produce certain work products. These process inputs and outputs, in turn, should have certain characteristics to meet capability requirements.

The Bootstrap model is under licence, which has prevented it from gaining such widespread use as CMM. Maintenance of the model was discontinued in 2003. 


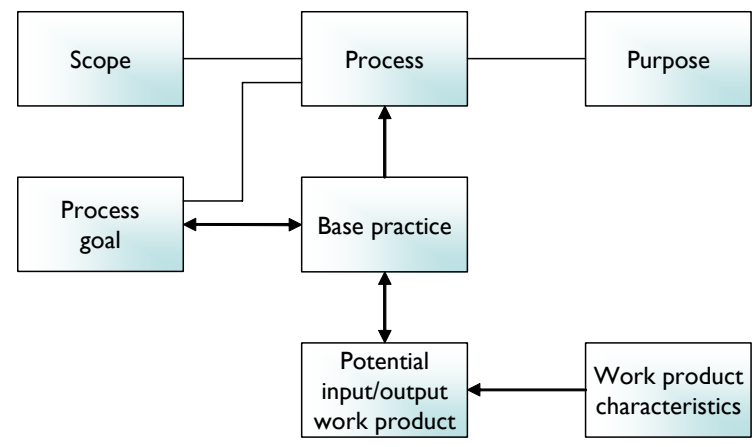

Fig. 22. The process dimension of the Bootstrap method. (Kuvaja et al. 1994)

As in most SPI models, Bootstrap introduces a lot of variables and cause-effect dependencies, making prediction of defect intensity and analysis of their root cause difficult (Fenton \& Neil 1999).

\subsubsection{ISO 15504}

The SPICE project was launched to create an international standard for process assessments. The first version of a draft standard was released in 1995. The goal of the SPICE project was to develop a standard that would be applicable to both process improvement and capability determination in different application domains. Furthermore, the standard was meant to be independent of organization size and structure, and of the methodologies used. Output was designed to be in the form of profiles instead of a single number or a pass/fail result (El Emam 1997).

In SPICE, the scope of the assessment is an organizational unit. The unit can be the whole organization or a part of it. The process context includes the application domain, the criticality, the complexity and the quality characteristics of the products or services that the organization produces. During the assessment, process instances are rated. For example, a particular project instantiates certain management and engineering processes. The assessment then produces a set of process profiles as an output.

The assessment typically includes four phases: preparation, data gathering, data analysis and feedback. When preparing for the assessment, organizational unit, process context and process instances have to be determined. The assessment scope does not have to include all processes. During the data gathering phase, process instances are investigated against the assessment model. Usually this is done by a qualified assessor. Data is collected from interviews and documentation relevant to the scope of the assessment. Automated tools, questionnaires or other instruments may be helpful. Two aspects of process instances are evaluated: what is done within the organization, and how well it is done. The assessor then proceeds to the analysis phase, in which process instances are given ratings. SPICE employs a four-level scale to describe whether a process fulfils its purpose: not adequate, partially adequate, largely adequate or fully 
adequate. This rating has to be done carefully and the assessor has to relate evaluations to the business goals of the organization. The evidence and justification for ratings are recorded to ensure that judgments are made objectively. The feedback and results of the assessment are formulated into process profiles. An assessment record is delivered along with the set of profiles to aid in understanding and interpreting the results. The profiles are usually spreadsheets or database records, which are typically aggregated and presented in graphical form to make the reports more understandable. The output of a SPICE assessment is used to highlight the organization's weaknesses and strengths for the planning of improvements.

The SPICE framework includes a reference model for process assessment. The reference model has two dimensions: the process dimension, which provides a strong mapping to the international standard for software life cycle processes (ISO/IEC 12207), and the capability dimension, which defines a measurement scale for the evaluation of the process.

The process dimension of the SPICE reference model consists of five process categories: Customer-Supplier, Engineering, Support, Management and Organization. These categories are described in Table 7. The capability dimension defines a six-level capability scale (from 0 - incomplete to 5 - optimizing). The higher the capability of the process, the less variation there is in its outcomes. Thus, it is more difficult to predict and estimate the outputs of a process with low-level capability. SPICE architecture identifies nine process attributes - characteristics of a process - that are evaluated to determine capability.

Table 7. The five process categories of the SPICE reference model (El Emam 1997).

\begin{tabular}{ll}
\hline Level & Description \\
\hline $\begin{array}{l}\text { Customer-Supplier } \\
\text { (CUS) }\end{array}$ & $\begin{array}{l}\text { Processes that have a direct impact on the customer and support development and } \\
\text { delivery of the software to the customer. Processes that provide for the correct } \\
\text { operation and use of the software. } \\
\text { Pngineering }\end{array}$ \\
(ENG) & $\begin{array}{l}\text { Pocesses that specify, implement or maintain a software product and its user } \\
\text { Support }\end{array}$ \\
(SUP) & $\begin{array}{l}\text { Poftware development. } \\
\text { Management }\end{array}$ \\
(MAN) & Processes that comprise generic practices used by anyone who manages projects \\
Organization & Processes that establish the business goals of the organization. Processes that help \\
(ORG) & the organization to achieve its business goals by developing products and services. \\
\hline
\end{tabular}

Processes can also be described as primary, organizational and supporting processes as in Figure 23. Each process category has a number of base practices attached to them. Examples of base practices are given in the figure. Joint review (not shown in the figure) is listed under supporting processes (SUP.6). Furthermore, there are a number of process indicators connected to the base practices. Indicators help the qualified assessor to gather evidence of whether certain practices are carried out in the organization (El Emam 1997). 


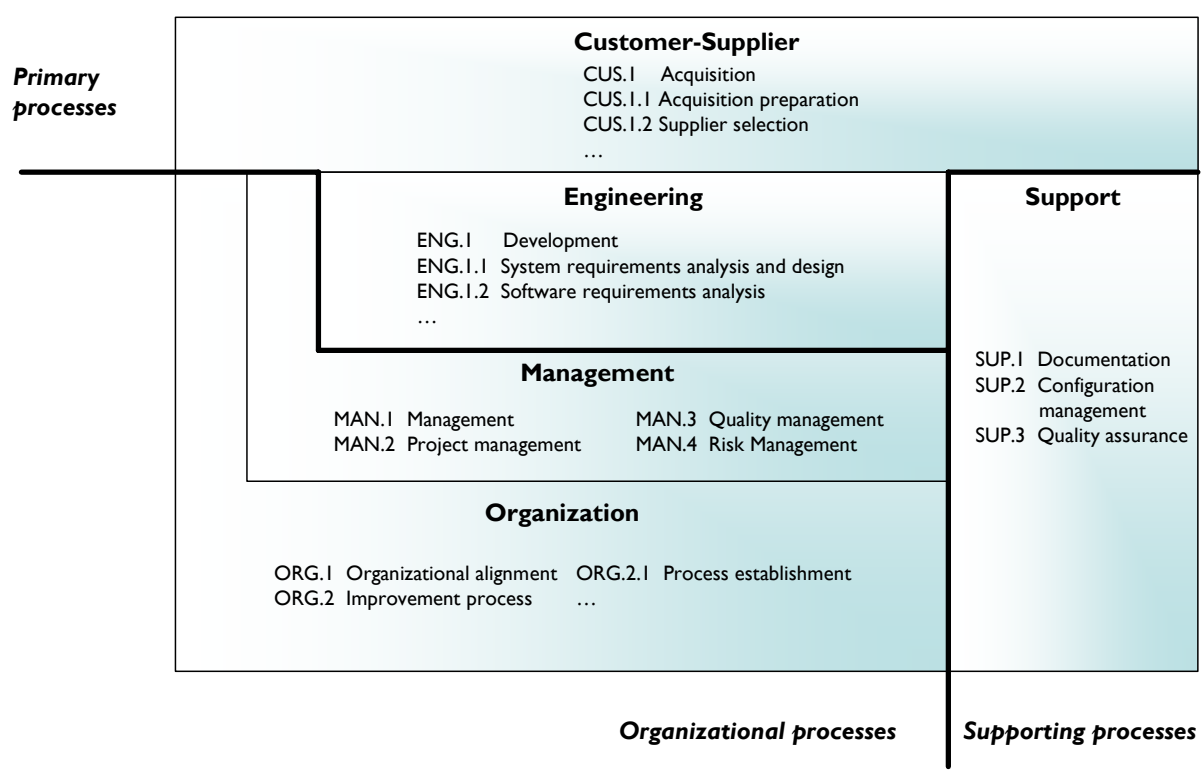

Fig. 23. ISO15504 processes (El Emam 1997).

Experiences of uses of the ISO15504 reference model for SPI have been reported by Simon (1999), for example. The article summarizes experiences in an organization where new business strategies had recently caused adjustments in development projects. As a result, Simon states that concepts and practices provided by standards can be adapted to the organization's needs. He also emphasizes the importance of planning and monitoring SPI efforts.

\subsection{The Role of Inspection in Generic SPI Models}

The role of inspection in generic SPI models has to be explored before the essence of inspection improvement can be defined. In this examination, the concept of inspection needs to be understood broadly, and the more general term "peer review" may be more appropriate to cover all inspection-related activities in the models.

Generic SPI models are not specific enough to cater to the needs of individual processes. Software inspection is categorized as a support activity, even though it plays a very important role in quality assurance.

The importance and value of inspections is pointed out in all full-scale process improvement models, although the inspection itself does not possess a process status in any of them. Rather than a process of its own, reviews are typically declared a supportive quality management task. For example, Bootstrap defines two types of reviews: joint review (SUP.6) and audit (SUP.7). Both are categorized as support processes, which, according to the Bootstrap specification, "may be employed by any of the other processes (including other supporting processes) at various points in the software life cycle." Joint 
reviews are performed from management, technical and customer viewpoints to ensure that the product satisfies customer needs. Audits are used to determine from the outside that products and processes comply with the defined requirements (Kuvaja et al. 1994).

It is true that reviews and inspections are supporting tools for quality assurance, but establishing, running and optimizing a formal inspection process, for example, involves many tasks and activities that need to be implemented carefully. Detailed process definition and instructions for the implementation would be beneficial.

As a supporting activity, peer reviews should be implemented at certain maturity levels. For example, in CMM, inspections are introduced at level three. However, the generic SPI frameworks provide very little information and guidance on exactly what documentation should be inspected, who is responsible for managing and arranging inspections, and when inspection should be arranged.

There is practically no research showing detailed information on how inspections have been initiated within large-scale process improvement programmes. Articles that report inspection installation successes and failures concentrate solely on the inspection method and do not take into account the larger SPI project, if such a project exists.

Generic SPI models can be enhanced with additional process descriptions, and further guidance on how to implement certain processes can be used in conjunction with the generic framework. The problem is that if new processes are defined, the resulting SPI model may become over specified. For that reason, enhancements or additional process models should be as easy-to-use and understandable as possible.

\subsection{Problems in SMEs}

Process improvement is as important (or even more important) in small companies as in large ones. To stay competitive, small companies have to produce high-quality products. Furthermore, many small companies work as subcontractors to bigger enterprises, which often require that their partners fulfil certain quality requirements: that they hold an ISO 9000 certificate, for example. However, the most prominent SPI models have many deficiencies concerning small organizations. Often the problems relate to the limited resources that small organizations can assign to the improvement effort (Buchman \& Bramble 1995, Geyres et al. 1997, Horvat et al. 2000).

The problem with full-scale process improvement models in smaller companies is that implementing the improvement programme requires almost the same effort regardless of the size of the company. Because the amount of personnel is smaller, training costs may be significantly lower, but other activities, such as establishing infrastructure for quality assurance functions, do not correlate to company size (Kelly \& Culleton 1999).

Generic SPI models do not provide adequate instructions for the implementation of SPI programmes (Johnson \& Brodman 1998). CMM includes practices that are difficult or impossible to accomplish in small companies. Independent software quality assurance (SQA) or configuration management (CM) functions, for example, are very difficult to establish with a limited number of people. The costs of a CMM-based improvement programme easily become too high for SMEs, as certain minimum levels of documentation and human resources have to be assigned to the improvement regardless 
of the size of the company (Brodman \& Johnson 1994). Paulk (1998) states that CMMbased improvement is achievable even with limited resources, but the model needs interpretation in small projects and companies.

In (Sakamoto et al. 1998), two problems with assessment-based approaches are pointed out, which may become especially challenging in small organizations:

1. SPI models give little information on how to proceed with the improvement.

2. The items that are assessed during an SPI initiative are discussed only at a generic level in the SPI models.

According to (Sakamoto et al. 1998) conducting process improvement requires a great deal of effort. SPI involves a great deal of information, which is unstructured and hard to manage. Convincing stakeholders to commit as necessary may be challenging. The lack of a shared goal and unclear roles of the people participating in the improvement may also cause problems. It is necessary to have concrete guidelines on how to implement all the required SPI activities, who should participate and what precisely should be done (Sakamoto et al. 1998). This viewpoint is backed in (Lepasaar et al. 2001), which states that small companies require a great deal of external expertise, resources and guidance for SPI.

According to Brodman and Johnson (1994), problems are amplified in smaller organizations, because they do not have the necessary resources and do not know where to turn for help. Even organizations that belong to a larger parent organization experience the same problems. On the other hand, changing the culture in smaller organization is easier once improvement has been accepted as a common goal.

Many small organizations work as subcontractors to larger companies. Subcontractors are often subjected to the prime company's standards and processes. Thus, the development model may vary from one project to another (Brodman \& Johnson 1994).

Project-level CMM practices (e.g., CM and SQA staffing, documentation) do not seem to apply when the projects are small (less than twenty people). When the whole development organization is small, there will be problems with organization-level practices as well (e.g., in-house training programmes). Furthermore, the cost of the SPI programme may be too high (Brodman \& Johnson 1994).

Small organizations can still follow the spirit of CMM. Successful SPI in smaller organizations requires software quality to be given an organization-wide emphasis and attention. Furthermore, a small company should have relationships with larger organizations with more mature processes, or other channels for the utilization of outside resources for process improvement (Brodman \& Johnson 1994).

SPI may take a considerable amount of time - even several years (Herbsleb \& Goldenson 1996). In their survey of CMM usage, Herbsleb and Goldenson report several issues that company representatives consider problematic. The most common problems are briefly summarized in Table 8 . The percentage in the table represents the portion of company representatives that recognized the problem. 
Table 8. Problems that companies have experienced with SPI. (Herbsleb \& Goldenson 1996).

\begin{tabular}{ll}
\hline Percentage & Problem \\
\hline $77 \%$ & Improvement takes longer than expected. Generally, it takes two years to move up one \\
& maturity level. \\
$72 \%$ & Time and resource limitations have caused setbacks to process improvement. \\
$68 \%$ & The cost of the improvement is higher than expected. Long-term resource allocation causes \\
& this. \\
$67 \%$ & The items that need to be improved are understood but knowledge on how to improve is \\
& lacking. \\
$57 \%$ & More individual mentoring and assistance is needed during the SPI.
\end{tabular}

Particularly in small organizations, real-life reports of successful process improvement in similar organizations would be beneficial. However, such research articles are rare (Kautz et al. 2000). SPI can still be achieved in smaller companies, and the usage of improvement frameworks in small companies has increased in recent years. In a recent study performed in Norway, it is reported that small organizations can implement improvements as effectively as large ones. However, the improvement strategy in small companies emphasizes the creativity and diversity of their human resources rather than strict formal procedures and rules (Dybå 2003). Another example of successful SPI in a small company is reported by Kelly and Culleton (1999). In their research, special attention is paid to cultural differences between small and large companies and the innovative and dynamic environment of small companies. Instead of maximum formality and CMM compliance, the improvement efforts are aimed at increasing quality (Kelly \& Culleton 1999).

There is a danger that small organizations attempt to gain improvement with shortterm solutions. Sheard and co-authors (2004) introduce the term "silver bullet of the month", by which they mean temporary improvements that are achieved by trendy methods or tools, or by pressuring individual developers to do a better job. Limited resources may result in arbitrary interpretations of, or cutbacks in the requirements defined by SPI models.

\subsection{Requirements for Software Inspection Capability Model}

Issues that SME companies consider problematic (documentation overload, management and SPI role issues, inapplicable process scope, high resource requirements, high training costs, lack of necessary guidance, etc.) need to be taken into consideration when introducing a light-weight improvement model for one process area. Herbsleb and Goldenson (1996) state that the factors that cause an implementation of an SPI programme to succeed or fail have been paid little attention in research. A number of experience reports can still be found in the literature that apply to outlining the required characteristics for the inspection capability model.

Otoya and Cerpa (1999) have studied a small company trying to improve its processes through a CMM-based tailored improvement model. Even though the improvements 
accomplished did not take the company to a higher level in the CMM framework, the initial improvement actions in the project planning and management areas had positive effects on other key process areas as well (Otoya \& Cerpa 1999). This suggests that focusing the improvement on certain practices can still benefit the whole development organization. Furthermore, Otoya and Cerpa report that the SPI knowledge that the management gained from the limited improvement was very beneficial for the company.

Wiegers states in his critical writing on software development models that instead of striving to match a certain process improvement model, one should be flexible and use the existing models as guidelines towards achieving better results in production. In his opinion, a practical approach is better than cumbersome and artificial methodology (Wiegers 1998).

According to Richardson (2002), the following characteristics are required from a SPI model for small companies:

- The model has to relate to the company's business goals.

- The focus has to be on the most important software processes.

- The model has to give maximum value for money.

- The proposed improvements should reach maximum effect in as short a time as possible.

- Return on investment should be fast.

- The model should be process-oriented.

- Relations to other software models should be defined.

- The model should be flexible and easy to use.

Rout (2000) suggests a rapid assessment method for measuring processes. The assessment is done in a single day to minimize the resources and costs required. To achieve reliable results in such a short time, it is crucial for the assessors to have adequate competence and experience. The scope of the assessment is limited. Data for capability determination is collected in moderated discussions, and ratings have to be agreed by all the participants.

In small companies, creativity must not be prohibited. Focusing on the project-specific problems instead of on processes may help to sustain the developers' productivity and inspiration. The participants should be involved but not disrupted by SPI activities. The main concern of improvement has to be that of software quality rather than strict compliance to a model, such as CMM. Tools can be utilized to ensure the efficiency and to enable the agility of the improvement. SPI initiatives ought to have an advisory role in the improvement (Kelly \& Culleton 1999).

Abbott (1997) identifies several notable requirements for achieving successful SPI in a small company. First of all, senior management support is crucial and adequate staffing for implementing the improvement must be ensured. This may be an issue when the organization is a small one. Project management principles should be applied to process improvement, meaning that objectives, schedules and resources need to be planned and tracked carefully. Integration with ISO9001 should be enabled, to allow future enhancements. Furthermore, assistance from process improvement consultants is beneficial. In general, the SPI effort should focus on providing real value to projects and to the business. 
Johnson and Brodman (1998) have addressed the challenges of small organizations and projects and introduced a tailored version of CMM, in which special attention is paid to the issues that are considered critical in such environments. Efficient handling of requirements and generation of documentation is important. Project management issues and resource allocations need to be considered, as there are no resources to waste. The progress of the improvement needs to measured, reviewed and communicated, to motivate further changes. Training is also considered a vital part of success (Johnson \& Brodman 1996, Brodman \& Johnson 1994).

Software process improvement activities have to be culturally adapted to each organization (Sakamoto et al. 1996). Because management hierarchy is simpler and communication is easy in small organizations, improvement frameworks should be scaled down to match the situation.

Dybå (2000) has derived six key factors for successful software process improvement from literature, expert opinions and real life case studies. These factors are:

- business orientation

- leadership involvement

- employee participation

- concern for measurement

- exploitation of existing knowledge

- exploitation of new knowledge

In addition, Dybå introduces indicator sets that can be used to guide and evaluate SPI actions. Indicators are linked to specific success factors and are rated on a scale of one to five.

Evaluation of the effects of the improvement is important. Höst (2000) reports an SPI effort involving code reviews, and suggests investigating improvement proposals and effects through interviews and experiments.

In (van Solingen 2004), two separate cases of improvement programmes are evaluated from the return on investment viewpoint. The benefits of SPI actions are clear in both cases, but the author stresses that special attention should be paid to the communication of improvements and improvement actions to all stakeholders.

According to Jacobs (2004), process improvement is expensive and burdensome in the present climate, as software development is fast-paced and costs should be minimized. She suggests accelerating SPI by ensuring management commitment, open communication, authority delegation, proactive project management and training, for example. Jacobs' article also provides a comparison of accelerated and traditional SPI methods, stating that even though institutionalization of the process is more likely to happen with traditional SPI models, agile SPI may produce less bureaucracy and faster return on investment, as the process is in place faster.

Natwick, Draper and Bearden (1999) report a lightweight process assessment method that has several benefits in terms of developing quality culture. Process assessment forces a project review from a CMM perspective, thus enabling improvements in projects and organization. Furthermore, team building and project awareness are improved, which increases developer motivation and productivity.

Table 9 summarizes some of the requirements that can be derived from reported SPI efforts and experiences. 
Table 9. Proposal for a lightweight improvement model.

Desired characteristics

Resource consumption should be low.

The model should be easily adapted to the organization's needs.

Assessment should be carried out in a short time and results should be available immediately.

The model should be compatible with the generic SPI models.

Concrete guidelines for implementing the improvement should be provided.

Improvement actions should focus on the most important practices and produce fast return on investment.

The characteristics listed in Table 9 will be used as a basis for the inspection capability and improvement model.

\subsection{Summary}

Software processes need to be maintained and improved, because technology and practices evolve with time. Furthermore, market requirements become more demanding all the time. However, fast-paced development cycles, rapidly changing products and uncertain market conditions cause problems for improvement, especially in companies that have limited resources. One approach to the management of this situation is to focus on the most valuable practices.

There are certain gaps in the generic SPI models that allow more focused, additional process improvement methods to be used in conjunction with them. In many cases, this is in fact necessary, as the full-scale models aim at providing a full picture of the development instead of specifying specific processes in detail. Because software inspection is among the most effective quality assurance methods when measured in terms of its return on investment factor, it is feasible for SMEs to begin process improvement from the software inspection process.

As Cunningham and Finnegan (2004) state, SPI concerns organizational information and different people's roles and responsibilities. Thus, the introduction of new procedures has to be methodical and well-controlled to avoid a reduction in effectiveness of the organization's infrastructure.

To introduce and establish the software inspection process in a manageable way, a framework guiding assessment and improvement is needed. 


\section{Patterns in Software Engineering}

The history of patterns dates back to the 1970s, when Christopher Alexander introduced the idea of formulating patterns from rules of thumb in architectural design. Proven solutions to common design problems were packaged into an easy-to-understand format to enable knowledge-sharing and communication.

Software patterns became popular due to the popularity of the book "Design Patterns: Elements of Reusable Object-Oriented Software" by Erich Gamma, Richard Helm, Ralph Johnson and John Vlissides (1994). Design patterns have been applied successfully in the industry and they are considered one of the most influential recent additions to the software engineering field.

Since then, patterns have been used in numerous domains: organizations, processes and teaching, for example. At present, the software engineering field uses patterns widely in software architecture and design, and describing software development processes and organizations with patterns has become increasingly popular.

\subsection{Defining Patterns}

The concept of general patterns was introduced by the architect Christopher Alexander. His discovery was that particular characteristics occurred repeatedly in modern buildings and architectural designs (Alexander et al. 1977, Alexander 1979).

There are several inconsistent definitions available for a pattern. Alexander's original definition is as follows:

Each pattern describes a problem that occurs over and over again in our environment and then describes the core of the solution to that problem in such a way that you can use this solution a million times over without ever doing it the same way twice.

One significant aspect of this definition is that it allows creativity even though a pattern states the overall structure of the solution. There is another definition by Alexander: 
Each pattern is a three-part rule, which expresses a relation between a certain context, problem, and solution.

Gamma, Helm, Johnson and Vlissides (1994) define design patterns as

...descriptions of communicating objects and classes that are customized to solve a general design problem in a particular context.

Riehle and Zullighoven (1996) give the following definition for the term "pattern":

A pattern is the abstraction from a concrete form which keeps recurring in specific non-arbitrary contexts.

This is a widely applicable definition. The concept of concrete form in this definition means not only software components, but any type of technical or non-technical entity. The context of a pattern is significant, because when the context changes, the forms of the entities change. Thus, patterns arise from the knowledge and experience of their domain and can be understood only within that domain (Riehle \& Zullighoven 1996).

Whatever the definition, the purpose of a pattern is to link together a problem, a context and a solution to the problem. Patterns are written in a universal and quite formal way, but at the same time they are practical and adaptable.

The variety of pattern types has increased. Design patterns, process patterns, organizational patterns, and pedagogical patterns are examples of areas taking advantage of the pattern approach. The use of patterns to improve development work in terms of productivity has been reported in Taylor (1998). Pattern languages have also been introduced to improve software usability (Mahemoff \& Johnston 1998), and documentation (Johnson 1992). Re-engineering is an interesting application domain of patterns, as in re-engineering both software patterns and process patterns can be utilized (Stevens \& Pooley 1998, Lano \& Malik 1997). Patterns have also been utilized in groupware application design to overcome difficulties in describing complex sociotechnical aspects (Herrmann et al. 2003).

\subsection{Alexandrian Patterns}

In the mid-1970s, Christopher Alexander introduced the idea of converting rules of thumbs in architecture into patterns and pattern languages.. Commonly-encountered design problems and solutions to these problems were packaged into reusable form in order to record and share knowledge of proven solutions. These patterns then provided a common vocabulary for the communication of ideas between experts and novices (Alexander 1979).

One example of the problem-solution pairings that these architectural patterns describe is the North Face pattern from the book "A Pattern Language: Towns, Buildings, Construction", (Alexander et al. 1977) listed in table 10. 
Table 10. The problem and solution in the North Face pattern. (Alexander et al. 1977)

\begin{tabular}{ll}
\hline Problem & Solution \\
\hline Look at the north sides of the buildings which you & Make the north face of the building a cascade which \\
know. Almost everywhere you will find that these are & slopes down to the ground, so that the sun which \\
the spots which are dead and dank, gloomy and & normally casts a long shadow to the north strikes the \\
useless. Yet there are hundreds of acres in a town on & ground immediately beside the building. \\
the north sides of buildings; and it is inevitable that & \\
there must always be land in this position, wherever & \\
there are buildings.
\end{tabular}

The architectural patterns proposed by Alexander are quite simple, yet very influential aspects of the layout of towns and buildings. The 253 patterns in the book form a pattern language, each pattern suggesting a solution to a certain problem. Patterns are interconnected and can be combined to achieve larger and coherent constructs, or a smaller set of patterns can be utilized to design a smaller environment. Furthermore, several patterns can be applied to the same space.

The sections of an Alexandrian pattern are not clearly segregated; the most notable syntactic structure is the conjunction therefore followed by the suggested solution. However, Alexander has expressed patterns in standardized format for convenience and clarity. An archetypal example of a specific pattern is illustrated with a picture, which is followed by an introductory paragraph. This sets the context for the pattern and explains how it helps to achieve certain goals. Next there is a headline for the problem, which provides the essence of the pattern in a couple of sentences. After the headline comes the body of the problem, which is the longest section. This describes the background of the pattern and the evidence for its validity. The solution is the most emphasized part of the pattern (written in a bold typeset), and it describes the physical and social actions that are required to solve the problem in that context. The solution is always stated in the form of an instruction to help the reader to understand what exactly is needed to build the pattern. Finally, there is a diagram illustrating the solution visually (Alexander 1977).

Alexander defines a pattern as a three-part rule that expresses the relationships between a certain problem, its solution and the context in which they apply. Furthermore, Alexander stresses the meaning of patterns as elements of language providing understandable instructions for the solution of real-world problems. Patterns can also be considered as process descriptions for the achievement of certain results in a given spatial arrangement.

According to Alexander, patterns are created for clarity, readability and comparability. This is achieved by consistent representation of patterns, even though they can address very wide-ranging problems. Some patterns are global (e.g., the Distribution of towns pattern) and some are very local (e.g., the Built-in seats pattern) (Alexander 1977). 


\subsection{Design Patterns}

The idea of design patterns originates from the late 1980s and user interfaces in the Smalltalk language. Ward Cunningham and Kent Beck developed some of Alexander's ideas to introduce a pattern language for guiding novice Smalltalk programmers. The resulting pattern language included only five patterns and was presented at the OOPSLA'87 conference (Beck \& Cunningham 1987). Reusing design and programming knowledge was the driving force for shifting the pattern concept to the field of software engineering (the paper's title was "Using Pattern Languages for Object-Oriented Programs"). The pattern approach was also taken advantage of in Jim Coplien's catalogue of C++ idioms, which was published as a book in 1991.

In Coad (1992), a pattern notation and seven patterns for object-oriented design and analysis are suggested. The article also provides a straightforward definition for an object-oriented pattern: An object-oriented pattern is an abstraction of a doublet, triplet, or other small grouping of classes that is likely to be helpful again and again in objectoriented development.

Patterns were addressed in later OOPSLA conferences, and the pattern activists started a pattern-oriented PloP conference in the mid 1990s. The Design Patterns book by Gamma and his co-authors was published in 1994, and it is still the most influential writing on patterns.

Various researchers have contributed to the area by introducing variations of patterns for specific application domains, such as communications and parallel processing. Refinements to existing patterns have also been introduced, as well as completely new patterns addressing new software engineering problems.

Design patterns are defined in (Gamma et al. 1994) as follows:

...descriptions of communicating objects and classes that are customized to solve a general design in a particular context.

Coplien (1995) gives the following definition:

Design patterns capture the static and dynamic structures of solutions that occur repeatedly when producing applications in a particular context.

The essence of these definitions has the same message as the original Alexandrian characterization of the meaning of "pattern": problem, solution and context information is captured, packaged, communicated and reused in a practicable form. At a general level, design patterns can be viewed as well-documented building blocks for software designs. When they are implemented in actual code, they constitute building blocks for entire software systems.

The structure of design patterns is fixed. All 23 patterns in the Design Patterns book are consistently represented and formatted. Each pattern has thirteen parts describing all the aspects of the pattern: its name, intent, aliases, motivation, applicability, structure, participating components, collaborating components, consequences, hints for implementation, sample code, known uses and related patterns. The elements of a design pattern are described in Table 11. Patterns are described uniformly to enable easy comparison, and judgment of whether certain patterns are appropriate in given situations. 
Table 11. The elements of a design pattern. (Gamma et al. 1994)

\begin{tabular}{|c|c|}
\hline Element & Description \\
\hline Pattern name & $\begin{array}{l}\text { Identifies the problem, its solution and consequences in a few words. Effective } \\
\text { naming of patterns increases the design vocabulary and makes developer } \\
\text { communication more effective. }\end{array}$ \\
\hline Intent & Describes briefly the purpose of the pattern. \\
\hline Also known as & Gives alternative names for the pattern. \\
\hline Motivation & $\begin{array}{l}\text { Gives examples of problematic situations in which the pattern can be utilized. The } \\
\text { problems may concern algorithms or class structures that indicate inflexible } \\
\text { design, for example. }\end{array}$ \\
\hline Applicability & $\begin{array}{l}\text { Describes the situations in which it is especially useful or appropriate to use the } \\
\text { pattern. }\end{array}$ \\
\hline Structure & $\begin{array}{l}\text { Explains the components and their relationships that make up the design; provides } \\
\text { an abstract representation without implementation-specific details to make the } \\
\text { pattern applicable in varying situations. }\end{array}$ \\
\hline Participants & Lists the classes and objects that constitute the pattern. \\
\hline Collaborations & Describes the external relationships of classes and objects within the pattern. \\
\hline Consequences & $\begin{array}{l}\text { Discusses the costs and benefits of applying the pattern, in order to help the } \\
\text { designer evaluate different alternatives and understand the impact of the pattern } \\
\text { on a system's quality characteristics. }\end{array}$ \\
\hline Implementation & $\begin{array}{l}\text { Gives examples and hints for effective implementation of the pattern. Also } \\
\text { outlines language-specific or other problems that need to be taken into } \\
\text { consideration when implementing the pattern. }\end{array}$ \\
\hline Sample code & Provides sample code to exemplify the pattern implementation. \\
\hline Known uses & Lists famous class libraries or applications, in which the pattern has been used. \\
\hline Related patterns & $\begin{array}{l}\text { Clarifies relationships between patterns, such as using the pattern together with } \\
\text { some other pattern or as an alternative for another pattern. }\end{array}$ \\
\hline
\end{tabular}

Patterns are classified into three groups: creational, behavioural and structural patterns. These address object creation, the functionality of objects, and the architectures of subsystems, respectively. Another way to categorize patterns is according to their scope patterns can be applied either at class or object level (Gamma et al. 1994).

\subsection{Examples of Other Software Development Patterns}

After the noteworthy success of design patterns in software engineering, Rossi, Schawabe and Garrido (1997) first presented design patterns for hypermedia. An example of a hypermedia-oriented design pattern is the Shopping basket, which keeps track of the user's selections during their navigation, separating the processes of selection and orderprocessing (Rossi et al. 1999). Many other aspects of hypermedia applications have been described with patterns. Hypermedia patterns can record very generic best practices in written format, or they can outline complex collaborative characteristics in hypermedia systems. German and Cowan (2000) attempt to unify and standardize numerous hypermedia patterns. 
Fredj and Roudies (1999) apply patterns to the requirements engineering phase of software development. They argue that the pattern approach provides a flexible way to assist developers in requirements elicitation and analysis. Patterns do not impose conventional bottom-up or top-down orderliness, but allow mixed approaches as well.

In (Buschmann et al. 1996), the pattern approach is applied to the description of entire software architectures. Architectural patterns can be seen as a generalized form of frameworks, providing abstract descriptions of systems independent of operating systems, programming languages, networks and database environments.

Examples of other adaptations of design patterns include pattern language for designing human-computer interaction (Borchers 2000), and Landay and Borriello (2003) suggest using design patterns for communicating and reusing development knowledge in ubiquitous computing applications.

In all these examples, patterns are utilized to create a common vocabulary for specific areas of software engineering and to increase the productivity of development by encouraging the documentation, communication and reuse of observed best practices.

\subsection{Process Patterns}

Most patterns and pattern languages introduced in research address problems that directly relate to software design and implementation. In addition, the pattern approach has been applied to organizational and process elements of the development.

Whereas design patterns embody practical solutions to recurring problems confronted in software design, process patterns provide help on how to efficiently carry out particular tasks during the development process. Thus, each process pattern should represent a proven and systematic approach to a given task. As with design patterns, process pattern users benefit from previous experience of implementing those tasks. In the same way as design patterns, process patterns emerge from practice, capturing the knowledge gained in software development into reusable and practical form. The formal identification and cataloguing of process patterns disseminates knowledge and focuses the efforts of the people involved in the development.

Process patterns were first introduced in Coplien (1995). After that, the concept has been developed by numerous researchers. Analysis patterns (Fowler 1997) are conceptual patterns that can be used to improve the conceptual modelling process. Huang \& Zhang (2003) provide exact guidance for modelling the roles, activities and workflow of the software development cycle with hierarchical process patterns. The patterns can be used to describe a development lifecycle, for example. (Moore et al. 2000) reports studies on the process pattern approach in order to model complex, domain-specific engineering tasks. Störrle (2001) describes workflows using UML-based process patterns. Cain, Coplien and Harrison (1996) have discovered in their studies that organizations often unconsciously adopt patterns when modelling processes. These organizational patterns are effective independently of the organization if they are abstracted suitably. An organization can evaluate the patterns and choose the most suitable pattern for its own domain. The use of organizational patterns has been tested in real AT\&T projects to validate Coplien's idea. Mann and Rising (2005) introduce a comprehensive pattern 
language comprising 48 patterns for dealing with resistance to change and other problems concerning organizational change.

Ambler introduces a three-category classification of process patterns in his books (Ambler 1998, Ambler 1999):

- A task process pattern describes the steps required to perform a specific task in detail. Task process patterns are key components of a software process.

- A stage process pattern describes the steps required to accomplish a single project stage. It is a higher-level pattern, typically composed of several task process patterns. Thus, stage process patterns organise and set the context of the task process patterns.

- A phase process pattern describes the relationships between the stage process patterns in one project phase, e.g., the delivery phase.

These patterns are aimed at defining and representing software processes. The pattern approach has also been suggested for improving business processes (Seruca \& Loucopoulos 2003), to increase understandability and to capture and promote organizational knowledge and best practices. (Benedicenti et al. 1997) provide an example of modelling organizational structures with patterns. The experiment was carried out in a small Italian company and Coplien's pattern language was used.

In (Gnatz et al. 2001, Gnatz et al. 2002) a template for representing process patterns is suggested. The elements of the template are described in Table 12.

Table 12. The elements of a process pattern as suggested in (Gnatz et al. 2002).

\begin{tabular}{ll}
\hline Element & Description \\
\hline Name & The name of the software development process pattern. \\
Also Known As & Other names for the pattern, if any are known. \\
Author & The names of the authors of the pattern. \\
Intent & A summary of the pattern's purpose and rationale. \\
Problem & The issue or problem that the pattern provides a solution for, including a \\
& discussion of the background of the problem. A real world example can be \\
provided to demonstrate the problem. & The situation or state of a development project in which the pattern may be \\
applied. External circumstances and influences can also be addressed in this & section. \\
The suggested work products and development activities within the process & pattern to solve the problem. The solution may be described in textual or \\
graphical format. & The potential benefits and liabilities that using the pattern may produce. \\
Kolution & $\begin{array}{l}\text { Known uses of the pattern in development projects. These application examples } \\
\text { demonstrate the usefulness of the pattern and provide hints for implementation of } \\
\text { the pattern. }\end{array}$ \\
References to patterns that solve similar types of problems and to other patterns \\
which help to refine this pattern.
\end{tabular}

Because process patterns do not impose how to perform a given task, they can be reused over and over again. They are building blocks enabling descriptions of tailored software 
processes for the specific needs of the organization. Process patterns are closely related to organizational patterns, which describe common management techniques or organizational structures.

For historical reasons, the expression "pattern" seems to involve object-orientation even though processes or organizations described with them may not be concerned with the development paradigm. Ambler (1999) states that "a process pattern describes a collection of general techniques, actions, and/or tasks for developing object-oriented software." Coplien (1995) does not emphasize object-orientation: "the patterns of activity within an organization (and hence within its project) are called a process."

Peer reviews have also been defined as process patterns. Ambler (1998) describes a Technical review task process pattern that depicts a high-level review process in pattern format. Ambler's process patterns include four sections: forces, initial context, solution and resulting context. In Table 13, the elements of the Technical review pattern are presented in a concise fashion.

Table 13. Technical review process pattern. (Ambler 1998)

\begin{tabular}{ll}
\hline Element & Description \\
\hline Forces & Validating artefacts before they enter subsequent development phases. \\
& Fixing defects is less expensive in early stages. \\
& Taking advantage of peers' expertise to validate products. \\
& Improving communication. \\
& The artefact and the review team are ready for the review. \\
The development team prepares for the review. & The team indicates its readiness. \\
Initial context & A cursory review is done by the review manager. \\
Solution & The manager organizes and plans the review. \\
& The review takes place. \\
The results of the review are acted on. \\
The quality of the artefact conforms to defined needs. \\
The team has a better understanding of the artefact. \\
Team members have probably learned new development or reviewing techniques. \\
\hline
\end{tabular}

This process pattern describes basically the motivation and running of a review process in an easy-to-follow format. The activities required by the pattern can also been visualized as a process diagram. Coplien's Review pattern and Harrison's Validation by Teams pattern also address peer reviews (Coplien 1995, Harrison 1996).

Patterns for software process improvement have been presented by Appleton (1997) in an attempt to overcome some typical difficulties in improvement efforts. Reducing resistance to change and targeting the improvement effort efficiently are the main focuses of these patterns. The process improvement pattern language includes ten patterns that are categorized as organization patterns or process and communication patterns. The patterns are chosen on the basis of their repeated success as documented in process improvement literature. Each of Appleton's patterns includes nine elements. The sections are: pattern name, context, problem, forces, solution, resulting context, rationale, related patterns and known uses. 


\subsection{Benefits of Patterns}

Patterns are independent of programming language and other implementation techniques, and therefore they have wide applicability. Pattern catalogues, such as (Gamma et al. 1994), have emerged from software engineering best practices, and their usefulness has been validated before publication (at least, in an ideal situation). The literature includes several experience reports that further prove the pattern approach.

Schmidt (1995) lists several advantages delivered by design patterns. Patterns enable widespread reuse of software architectures. Reusing entities larger than algorithms and data structures is more valuable in terms of productivity. Patterns also improve communication within and across development teams, as problems can be addressed with a common vocabulary. For example, design alternatives can be communicated with the aid of patterns. Finally, patterns can be used to capture implicit knowledge in an explicit form (Schmidt 1995).

The knowledge and standardized design vocabulary aspects have also been recognized in (Wang et al. 1997), reporting that design patterns are useful in instructing novices on how to design reusable and flexible object-oriented software. Software quality especially reusability - can also be improved with patterns (Wang et al. 1997). Salzmann and Maraist (1997) also state that the use of design patterns enables flexibility and extendibility within software architecture.

The article (Beck et al. 1996) summarizes the key benefits that software organizations have observed from pattern usage. Improved team communication was seen as the most important advantage. Another considerable characteristic of design patterns was that they capture essential parts of a design in a compact form and that this essence is extracted from an existing, working design. Thus, patterns can encourage the reuse of best practices (Beck et al. 1996).

\subsection{Patterns as a Knowledge-Sharing Tool}

The knowledge-sharing aspect of patterns needs to be paid special attention when evaluating the benefits and shortcomings of patterns. Even though the abstractions of application structures that have been represented with design patterns have obtained the main focus in the pattern community, software patterns were first devised to educate novice programmers and provide them real-world, proven solutions. The main idea of the original Alexandrian patterns was also the transfer of knowledge from experienced designers to less experienced ones. In fact, the original objective of Alexander's work on pattern languages was to enable ordinary people to design their own homes (Alexander et al. 1977). This was definitely too idealistic and ambitious a goal, but the book certainly helped architects' clients to express their requests and requirements for a building more clearly. This is a significant shift of control, and it helped in detecting flaws in designs, and enabled clients to understand their environment.

However, patterns are useful in a more general business context, rather than just software engineering. The large number of organizational, educational and other process 
patterns already suggests that the approach is considered adaptable to any purpose that involves communicating proven solutions from experts to beginners.

May and Taylor (2003), for example, state that patterns can be used as a generalpurpose knowledge management tool. They have observed that using the pattern approach for knowledge management yields a high long-term return on investment. Furthermore, patterns provide a straightforward and intuitive technique for making tacit knowledge explicit.

\subsection{Problems with Patterns}

Practical use of any tool requires recognition of its limitations. The literature and experience reports identify several problems that may be encountered when utilizing patterns. Even the design pattern descriptions (Gamma et al. 1994) list some pitfalls that may be faced with individual patterns. The problems described here, however, relate to patterns in general.

Natural language is not necessarily sufficiently precise for the description of implementations. Sample code and structure sections provide concrete code extracts and UML diagrams of the solution, but other parts of the pattern description are written in natural language. The use of natural language is both an advantage and a shortcoming. Knowledge of specific notation or syntax is not required, but on the other hand there are considerable limitations in precision. Thus, ambiguous interpretations of the solutions presented in patterns are possible. This leads to confusion in recognizing how patterns work and how they are related to each other. There are also some similarities between patterns and sometimes it is difficult to recognize which pattern is in use in a certain piece of code. In the worst case, the wrong pattern is chosen for a particular situation.

Although practicability is perhaps the most looked-for characteristic of patterns, there can be certain difficulties in their practical utilization. Patterns are always abstractions of real-life problems, and if the chosen level of generalization is too detailed, the patterns will become unwieldy. If they are too abstract, they do not provide any concrete aid for the user. Furthermore, patterns dealing with new technology may cause difficulties.

There are some disagreements within the pattern community concerning what constitutes a pattern. A pattern can embody general and widely accepted knowledge about the given domain or it can introduce a new technology for doing something. For example, novice developers would benefit more from trustworthy, universal patterns, while experienced software engineers would probably appreciate novel ideas more (Dewar et al. 1999).

Furthermore, in the reengineering pattern domain, it may be difficult to persuade organizations to provide adequate information about their products to document pattern usage. This makes writing the "known uses" section especially challenging (Dewar et al. 1999).

The experiences reported in (Beck et al. 1996) about applying design patterns at several software organizations, including Motorola, AT\&T and Siemens. At Motorola, the difficulty of writing good patterns is seen as the major disadvantage of the pattern approach. To produce patterns that are adequately mature to be really useful also requires 
a considerable effort. Quantifying the impact of design patterns on software quality is also problematic, as the impact of patterns and that of other changes in the development process cannot easily be distinguished. Experiences at AT\&T show that patterns are considered illuminating and inspirational, but the number of patterns that have been produced has caused problems. Reports from Siemens state, similarly to Motorola, that writing good patterns may be exceedingly difficult. According to experiences at Siemens, the acceptance of patterns and the level of pattern application can be improved if a significant portion of the design is covered by either a set of low-level design patterns or a single high-level architectural pattern (Beck et al. 1996).

Even though design patterns provide practical and widely applicable solutions, using patterns in object-oriented development is not trivial, especially for novice programmers. First, the need for a certain pattern has to be identified by recognizing a problem and deciding on a particular solution. Then the chosen pattern has to be integrated with the design that is already available. It may be necessary to change the existing design of a software program to fit the pattern into it, or the pattern itself may need to be adapted to fit the existing architecture.

Due to their recent success, the use of design patterns can be over emphasized. Instead of designing the best possible solution, novice developers may focus on using as many design patterns as possible, leading to an unnecessary complex implementation. The suitability and selection of a pattern should be evaluated carefully on a case by case basis (Buschmann et al. 1996).

Patterns provide little support for actual implementation. Generic patterns must be mapped to concrete implementations each time they are used. Furthermore, patterns can be implemented in different ways, making it difficult to recognize whether they are being used correctly. Soukup (1995) notes the problem of poor traceability of patterns within large application architectures. The problem may even be interpreted differently by individual developers and different patterns may be used to solve the same problem (Soukup 1995).

Finally, the number of different patterns for different domains is increasing all the time. Since there are several pattern languages and formats, creating a common patternbased vocabulary becomes impossible. Encapsulating experience in patterns and, likewise, searching through all available patterns to reuse an existing solution, becomes too laborious (Agerbo \& Cornils 1998).

All these shortcomings of patterns mainly concern design patterns. Critical experience reports on process and other kind of patterns are very rare. Most of the problems listed here also have potential solutions to them, and the expected benefits of design patterns outweigh the shortcomings.

\subsection{Summary}

The use of patterns for the storage and transfer of expert knowledge originates from the field of architecture in the 1970s. During the 1990s, the pattern approach was successfully adapted for software engineering purposes to capture proven design solutions, especially in object-oriented domains. The resulting software patterns are 
literary forms that describe practical how-to methods for solving recurring problems in software engineering.

The limitations of patterns reported in different organizations relate to the difficulties of introducing change, supporting the usage of patterns, writing good patterns and measuring the impact of patterns.

When creating patterns, one has to choose the level of abstraction to use in the pattern carefully. A too general level does not provide adequate guidelines for practical implementation of the application or process structure defined by the pattern, and on the other hand a too detailed description hinders the applicability of the pattern across different organizations or projects.

In summary, it can be stated that the pattern approach has been observed to be a suitable technique for a variety of situations where rather complex activities or constructions need to be created. In general, patterns describe a problem, its context, and a solution to the problem. However, not any solution can be defined as a pattern. The problem addressed with a pattern should be reoccurring and the overall shape of its solution should be similar each time. Furthermore, to enable learning and efficient communication, patterns need to be named and explained carefully. 


\section{The i3 Model}

The i3 capability model is based on the ideas presented in generic software process improvement models. The i3 model attempts to capture the essence of the software inspection process and elements related to it. This is achieved by analysing the variations of software inspection methods and identifying the most fundamental activities that are required to carry out inspections in an efficient way. The work products that are produced in different inspection processes are also studied and included in the model.

The aim is not to produce another variation of the inspection process. Instead, the i3 model can be used to describe and define several types of inspections and to prioritize inspection activities according to the goals of an organization.

A model for assessing and improving software inspections is needed to aid in establishing the inspection process. For example, inspections were adopted in HewlettPackard in four stages: experimental, initial guidelines, widespread belief and adoption, and standardization, and it took several years before the method was fully adopted in the organization (Grady \& van Slack 1994). Introducing inspections in an organization that has no previous experience of the method is difficult.

The i3 model provides the means for planning and gaining sufficient understanding of the inspection process when introducing the method. Furthermore, the model can be utilized when identifying potentially ineffective areas in the process.

\subsection{Related Research}

A model for assessing the capability of certain sub-processes of software development is not a unique idea. A few models have been developed to enable test process assessments within an organization. Test Process Improvement (TPI) and the Testing Maturity Model (TMM) in particular have been comprehensively published and tested in research literature. Experiences from those efforts can also be used in creating an inspection capability model.

The TPI model (Koomen \& Pol 1999) presents a set of key areas and the practices connected to them. The practices are organized using test process maturity levels. TPI is not intended to be CMM-compliant. The structure of the TPI model is different from that 
of the CMM, which may result in difficulties if an organization that has already adopted the CMM approach wishes to apply the Test Process Improvement model.

The TPI model consists of a maturity model, a maturity matrix, a checklist, and improvement suggestions. The model has three maturity levels: controlled, efficient and optimizing. Aspects of testing are covered by twenty key areas that mainly concern system and acceptance tests. Each key area is rated with the aid of checklists and then the maturity matrix is used to define the improvement actions. The matrix is helpful in identifying dependencies between key areas, and targeting the improvement actions. It is not always sensible to improve all key areas. Improvement suggestions provided by the model are not compulsory steps of TPI, but hints for improving certain key areas.

The TMM (Burnstein 2003) is a staged maturity model, and can be used in conjunction with the CMM or CMMI. The goals defined by the Testing Maturity Model are achieved by activities, tasks and responsibilities that are structured by three critical views: manager, developer, and user/client. Unlike CMM, the TMM does not decompose the test process into key process areas. These structural differences can hinder use of the TMM and the CMM together.

The TMM model uses a questionnaire instead of checklists to determine the current status of testing practice. As in the CMM, the TMM defines five maturity levels. For each level, a number of goals are defined. To satisfy a certain level, the testing process needs to address all the goals defined for that level.

There are also maturity models for other software engineering processes. For instance, the reuse capability model enables the assessment of the level of reuse in the development organization (Davis 1993). A model to determine the capability of the requirements engineering process is represented in (Sommerville \& Sawyer 1997).

Rodgers and Dean (1999) have conducted a survey investigating the effects of inspection process maturity and reviewers' expertise on inspection results. The key findings were that both of these aspects affect inspection outcomes. Furthermore, they suggest that "process maturity should be assessed based on inspection practices within key processing areas in a manner similar to CMM." The article does not present more concrete ideas about CMM-based inspection maturity assessment.

\subsection{Overview}

The i3 model is designed to conform to the requirements summarized in Chapter 3.2. The first characteristics that are considered important are lightness and flexibility of the model to enable its effortless adaptation across organizations.

Research results suggest that if SPI activities are considered as a burden, the improvement will probably fail. The software process should help people within an organization to do their jobs more efficiently, and these people should have positive feelings towards the SPI. If the organization is small, a lightweight method has to be used to introduce improvements, to avoid overload. In addition, the organization and workers should be able to retain familiar work practices, and new tools, standards or procedures ought not to be imposed from outside. 
Thus the i3 model lists the features of an effective inspection process and provides guidance on how to achieve such a process, but the model does not enforce any fixed set of practices. An organization can choose the most feasible form of inspection and determine the direction of improvement actions according to that company's overall business goals and standards. As Porter and Votta (1997) state, there is no single correct process model for software inspection. Context and inputs are more important than process structure.

Adaptation of the inspection process is supported by research. For example, Grady and van Slack (1994) state that it is difficult to start doing inspections with an optimal process. Understanding organizational limitations and long-term plans, and addressing immediate needs is crucial for successful implementation of the inspection process. Davis and Royce (1994) suggest that instead of investing large amounts of money to establish a comprehensive inspection process, focused alternatives of the review process can produce reasonable gains at low cost and risk.

The i3 model provides a framework for defining the components of an organization's inspection process, assessing the current status of review activities and outlining the improvement actions.

To describe all the fundamental elements of inspections and to enable modularity, the i3 model is composed of process elements, called activities, and the products that these activities produce, called indicators. The basic structure is quite similar to the generic process improvement reference models, to allow easy transition to full-scale process improvement.

The assessment is as lightweight as possible and is carried out during one interview session. The results are available immediately and the initial objective for improvement can be discussed after the interview. The interview also provides an instructional forum: the company representatives have to communicate and agree on how, when and by whom review tasks are to be done now and in future. Furthermore, the i3 framework supplies a common vocabulary for discussing inspection activities. The framework can also be used to introduce elements that are currently missing in the organization's process.

Concrete instructions and hints for process improvement are provided by the improvement patterns. The patterns are easy-to-follow action lists that describe straightforward steps for the achievement of a more efficient process.

In summary, the i3 model aims to capture the essence of the inspection process by means of activities that have to be carried out to accomplish an efficient reviewing practice. These activities can be implemented differently between organizations, and some activities may be considered unnecessary in certain contexts.

\subsection{Elements of the i3 Model}

The i3 model is based on the ideas and structure of the Bootstrap methodology (Kuvaja et al. 1994). The model forms the basis for software inspection process assessment by defining the process as precisely as possible.

Inspection is traditionally defined in terms of process phases such as entry, planning, kick-off meeting, individual inspection, logging meeting, edit, exit and follow-up. A 
number of variations on these steps have been presented, as shown in Chapter 2.1. These steps also form the structure of the $\mathrm{i} 3$ inspection process model, but instead of breaking the inspection process down into consecutive phases, the i3 model aims at capturing the fundamental functions of the process and pieces of information that are passed between the stages.

The i3 model consists of two types of essential elements: activities, that describe the phases of the inspection process, and indicators, that describe the information flows between activities. The corresponding Bootstrap terminology would refer to these as base practices and work products.

The overall structure of the $\mathrm{i} 3$ model is depicted in Figure 24. The activities are depicted as ellipses. Activities are divided into three categories: core activities, organizational activities and supporting activities. This categorization corresponds to the grouping of base practices into primary, organizational and supporting processes in the ISO15504 model.

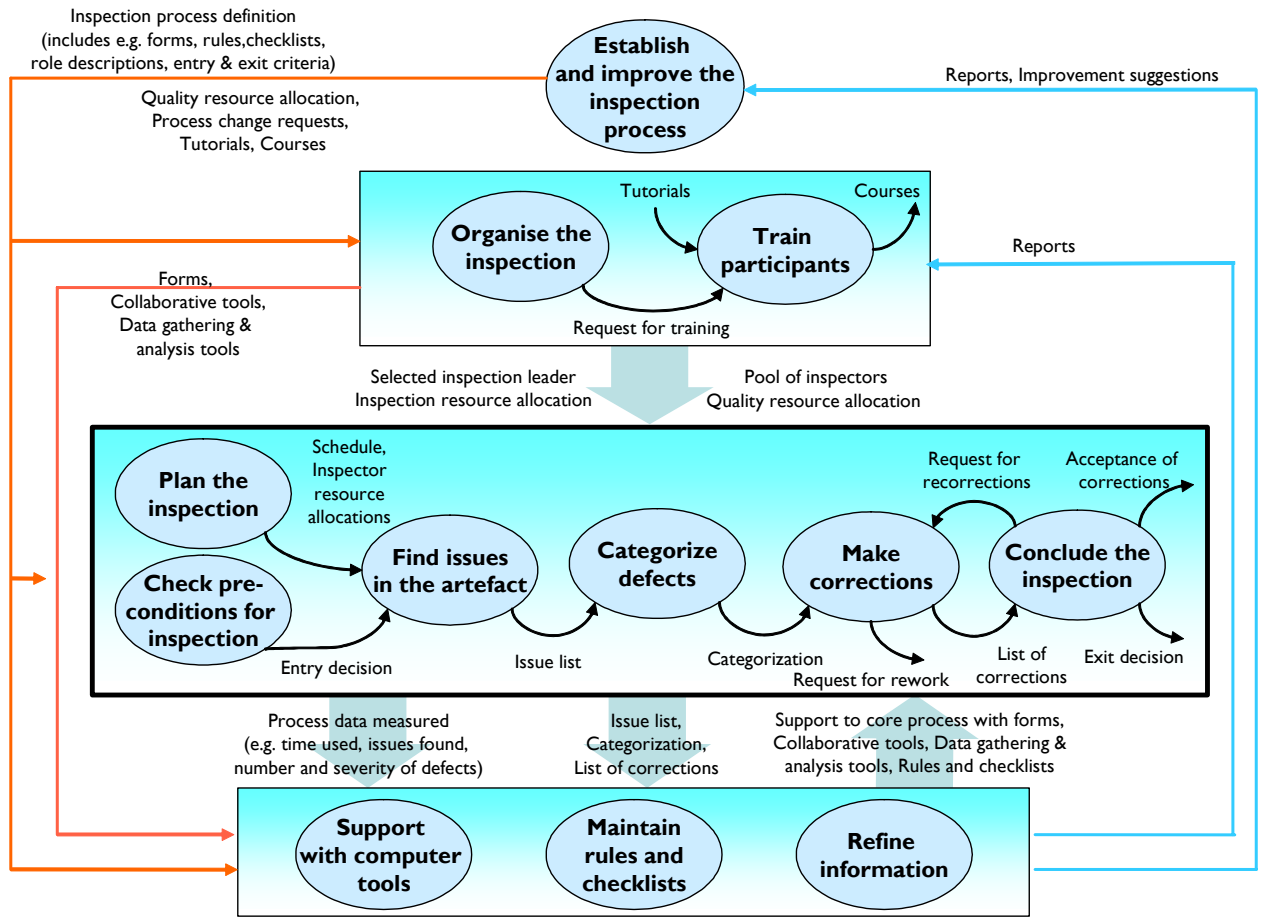

Fig. 24. The activities and indicators of the $i 3$ model.

In Figure 24, organizational activities are the three uppermost ellipses, core activities are in the middle of the figure, and the three lowest ellipses are supporting activities. Arrows between the activities illustrate the work products and information (the indicators) that are exchanged between the activities. 
According to Laitenberger (2001), complete description of an inspection process has to address five dimensions, which are: technical, managerial, organizational, assessment and tool support. "Organizational aspects" concerns the number of participants in inspections and the structure or timing of meetings, for example. "Technical aspects" means that the inspectors should be provided with adequately defined procedures and techniques to review software documentation. Typically, developers are used to writing code or creating designs, but for inspections they need guidance on how to read the same type of documentation. Both of these aspects are important, as providing training for a poorly-defined inspection process is difficult. The "managerial" and "assessment" dimensions concern the effects that inspections have on individual projects or on the organization. The "tool support" dimension describes the support that is provided for inspectors and inspection leaders to help them carry out the process efficiently.

These dimensions, as well as the components of different inspection processes (as shown by the examples in Chapter 2) show either directly or implicitly in the i3 structure. Distinct process models for inspection provide mostly for the core activities. Textbooks on peer reviews and reported research on inspection successes outline the fundamental characteristics for organizational and supporting activities.

\subsubsection{Activities}

The basic element in the structure of the $\mathrm{i} 3$ model is the activity, which is a procedure carried out during one inspection iteration. The inspection process is a set of procedures, in which these activities are carried out. A single activity does not directly correspond to a particular process stage.

An efficient inspection process should contain all the defined activities. However, the same activity can appear very different in different process implementations. Especially in smaller companies, certain activities may be informal in order to make the overall process more straightforward. Implementation of each activity can be adapted to the organization's needs. For example, an interpretation of an inspection tool in the "support with computer tools" activity may be very different in different cases. In a lightweight process a tool may be one simple spreadsheet, in a more comprehensive environment there may be a repertoire of tools including groupware for scheduling the process, managing checklists and so on.

Table 14 summarizes the activities defined in the i3 model. Activities 1 to 3 are organizational activities, activities 4 to 6 are supporting activities, and activities 7 to 12 are core activities. 
Table 14. Activities in the i3 model.

\begin{tabular}{llll}
\hline Activity id & Activity name & \multicolumn{2}{l}{ Activity id Activity name } \\
\hline A.1 & $\begin{array}{l}\text { Establish and improve the inspection } \\
\text { process }\end{array}$ & A.7 & Check preconditions for inspection \\
& Organize the inspection & A. 8 & Plan the inspection \\
A.2 & Train participants & A.9 & Find issues in the artefact \\
A.3 & Support with computer tools & A.10 & Categorize defects \\
A.5 & Maintain rules and checklists & A.11 & Make corrections \\
A.6 & Refine information & A.12 & Conclude the inspection \\
\hline
\end{tabular}

Activities are related to each other. Most of them produce outputs that are taken advantage of during other activities (that is, used as inputs). These documents, reports and other sorts of work products are tracks that can be used to trace the activities that are really performed during an inspection iteration.

In the following, the twelve activities of the $\mathrm{i} 3$ model are presented, and compared to the generic goals of software inspections summarized in Chapter 2.

\subsubsection{Establish and improve the inspection process}

The basic idea behind any software process model or software process improvement model is that the underlying process must be well-defined. The process definition makes software development more manageable, measurable and predictable (Humphrey 1989. The purpose of this activity is to establish and ensure the continuous measurement and improvement of the inspection process.

According to Wiegers (2002), a documented and practical review process is essential. Radice (2002) states that introducing inspections is easier with clearly defined processes. The process structure does not necessarily need to follow a fixed model - such as Fagan's - but the process needs to be predefined.

MacFarland (2001) lists several points indicating that coordination and planning are required in order to establish and improve the inspection process successfully. Users of the process need to have active roles in the improvement, and possible benefits should be communicated to all target groups. Using consultants and local champions help in achieving improvements.

The effectiveness of the inspection process itself can be measured, and the data can also be used to analyse and improve the development process. Gilb has included an optional process brainstorming meeting in the inspection process in order to capture ideas for development process improvement and the analysis of root causes of defects (Gilb \& Graham 1993).

Senior management commitment and a quality culture are crucial to making inspections an everyday practice. A person responsible for the process improvement and an owner for the entire inspection process have to be clearly defined (Grady \& van Slack 1994). 
When compared with the overall inspection goals, this activity directly serves the process improvement aspect. The more effective the inspection process, the more defects it reveals. Thus this activity has indirect effects on product quality as well.

Table 15 gives examples of literature references that point out the importance of an established process reviewing process and its continuous improvement. Typically, the inspection process is adjusted after initial experiences. Articles concerning the introduction of the method frequently emphasize the importance of organizational support in terms of allocating resources to inspections and monitoring their performance with a basic set of metrics. Without clear ownership and a recognized status within the development, inspections may be easily ignored.

Table 15. Literature sources for the "Establish and improve the inspection process" activity.

\begin{tabular}{ll}
\hline Reference & Notes \\
\hline (Grady \& van Slack 1994) & $\begin{array}{l}\text { Organizational commitment to the inspection process, updating inspections } \\
\text { and linking the inspection process to overall process improvement are } \\
\text { emphasized in this article. }\end{array}$ \\
A checklist for installing inspections is provided. For example, assessing the \\
organization's readiness for inspections and using champions as change \\
agents are suggested, to ensure successful adoption. \\
Management support is considered crucial in running the inspection process \\
successfully. Furthermore, the inspection process should be integrated into \\
other development to make inspections a standard procedure. \\
Inspections without a systematic process may be beneficial, but a defined \\
process, effective reading techniques and process optimization help in \\
(Ciolkowski et al. 2003) \\
successful inspection implementation. \\
Analysis of inspection data showing that even a small metrics set can help \\
considerably in optimizing inspection efficiency.
\end{tabular}

The results of an experiment in a small software development company (Harjumaa \& Tervonen 2000) confirm the significance of having a defined process. Even though resources can be saved with a lightweight method and tool support, the underlying process for inspections needs to be defined for successful implementation.

\subsubsection{Organize the inspection}

The effectiveness of the inspection is very much based on its formality. Even though different types of inspections exist and they can be carried out with varying levels of rigorousness, the inspection process should always involve strictly defined roles for participants (such as leader and inspector), and rules for performing the actual reading, and a specific flow of actions to be followed. Thus, the inspection should always be a well-organized occasion.

Establishing and maintaining organization-level resources for the inspection process requires that the process is precisely defined in the organization's quality handbook and 
actively promoted to ensure its everyday use. The necessity of and resources for training also have to be decided at the organizational level.

The purpose of this activity is to ensure that there is a person or group in the organization who is responsible for the process as a whole. The inspection process should be kept up-to-date and integrated into other development processes. Motivating the management as well as the people participating in inspections is crucial, in order to ensure coherent and organization-wide commitment. Success stories have to be reported to make clear the value of the process.

Management should not be too involved in inspections other than in promoting their usage. This ensures that developers do not feel that inspections are being used to evaluate their personal characteristics (Gilb \& Graham 1993).

Managing the inspection process and coordinating it with other project tasks is important, but it is surprisingly rarely addressed in inspection literature (Chatzigeorgiou \& Antoniadis 2003). It seems that the planning of review tasks is not usually done well before all other project activities. To ensure that inspections are included in every phase of the development and given equal importance to other development tasks from the project management viewpoint, a high-level inspection management and organizing function is necessary, that holds the ownership of the inspection process and relates it to the project's master plans.

Examples of literature references for this activity are listed in Table 16. Integration of the inspection management activities into other development management is seen as crucial. According to the research, the success of inspections is not dependent on the details of a process model as such, but on the precise following of the process.

\section{Table 16. Literature sources for the "Organize the inspection" activity.}

\begin{tabular}{ll}
\hline Reference & Notes \\
(Chatzigeorgiou \& Antoniadis & $\begin{array}{l}\text { This article presents empirical data showing that inspection planning and } \\
\text { (Kelly \& Shepard 2002) }\end{array}$ \\
& $\begin{array}{l}\text { The authors have performed three inspection experiments. The results } \\
\text { suggest that inspection techniques should be defined and based on generic } \\
\text { goals, the context, and the experience of the inspectors. Inspections should } \\
\text { be integrated into the development process. } \\
\text { An experience report that suggests sticking to a rigorously defined process } \\
\text { to get the most out of inspections. } \\
\text { Inspections should be based on defined and planned processes that should } \\
\text { also be measured to enable continuous improvement. Preparation times, } \\
\text { document sizes and guidelines for re-inspections are among the issues } \\
\text { considered. } \\
\text { The inspection process needs to be strongly supported by the management, } \\
\text { to motivate authors and inspectors and to provide them with the time and } \\
\text { resources to fix defects. }\end{array}$ \\
\hline
\end{tabular}

This activity is connected to the previous one, "establish and improve the inspection process", as without rigorous management of the process, its effectiveness cannot be measured or improved. It is also notable that this activity emphasizes the organizational 
management of the inspection process, which is a separate task from planning a single inspection iteration.

\subsubsection{Train participants}

The human factor is an important aspect of the inspection process. The success or failure of running the process is largely dependent on its participants. People involved in the review should be willing to and capable of performing their tasks. The purpose of this activity is to ensure that at all times there are people in the organization capable of carrying out and managing inspections.

A sound infrastructure is required to support the implementation of an inspection process. This means adequate training and documentation, and a supportive environment. Training is necessary but not sufficient to ensure widespread inspection usage (Grady \& van Slack 1994).

Attitude plays a significant role in effective inspections. The unavoidability of defects has to be recognized and accepted. Developers may then accept defect-detection aids, such as inspection, more easily. Active advertising of inspections increases their usage (Russell 1991).

In addition to motivation, inspectors and inspection leaders need knowledge of the process. According to Basili et al. (1996), proper implementation of an inspection process requires an accurate understanding of the tasks, organizational context and roles of those conducting the inspections. Training is essential in making inspections effective (Russell 1991, Ackerman et al. 1989). Fagan states that "people have to be taught or prompted to find errors effectively" (Fagan 1976). Furthermore, various steps in the process itself, as well as the use of computerized tools, have to be taught. Most inspection method variations are quite formal, and guidance is needed on checklist usage, logging meeting procedures and optimal reading paces, for example.

Working in different roles and utilizing perspectives, checklists and other reading techniques is easier after training, and the inspection leader role is especially important (Petersson 2001, Dunsmore 2002, Gilb \& Graham 1993).

When team members do not completely understand the review process, a great deal of effort will be required to achieve a common vocabulary and follow the process consistently. Documentation of the why, when, how and who of the review is required, and the training of participants is particularly important (Wiegers 2002).

Even though novice reviewers can learn from experts during inspection meetings by observing their work, separate courses and instructions are needed to guide individual inspectors. Training is recognized to be a more cost-effective method for education than in-process observation (Deming 1982, Humphrey 1989).

Table 17 summarizes some literature references that highlight the value of training. The articles show that both inspectors and inspection leaders need training on the method itself. Furthermore, if roles, perspectives, tools or other enhancements are in use, additional training may be required. Management may also need instructions on what is expected from them, if the method has not been used previously in the organization. 
Table 17. Literature sources for the "Train participants" activity.

\begin{tabular}{ll}
\hline Reference & Notes \\
\hline (Grady \& van Slack 1994) & Providing adequate training on inspections is seen crucial in achieving wide- \\
& spread use of inspections. \\
(Bisant \& Lyle 1989) & The researchers have experimented with a two-person inspection method. \\
& Training and education of both managers and inspectors is required for the \\
& correct performance of inspections. The researchers even present the idea of \\
& including inspections in the undergraduate curriculum. \\
& Task training can improve individual inspection performance and defect- \\
(Votta 1993) & Detection skills. \\
(Chowdhury \& Land 2004) & defects. In addition to expertise, the process has an influence on inspection \\
performance. & An empirical study in which the inspector's task expertise is named as the \\
key element in effective software inspections and training as one of the key \\
methods for improvement of inspection performance. Training is seen as \\
crucial to the improvement of individuals' expertise.
\end{tabular}

Training is perhaps the most easily identifiable activity within the i3 model that is not explicitly visible in any inspection process models. There are numerous examples in the literature emphasizing that adequate knowledge of the method increases inspector motivation as well as the productivity of the method. On the other hand, many inspection experiments assume that the observed subjects possess equal levels of knowledge, which is very difficult to verify. Finally, inspections require both task expertise and domain-area knowledge, which should be taken into consideration when planning for training.

\subsubsection{Support with computer tools}

A number of software tools have been introduced to ensure efficient inspection implementation in a variety of challenging situations. For example, when the inspection team is geographically distributed, tools can be used either to arrange a virtual inspection meeting (van Genuchten et al. 1997) or to collect inspector comments (Votta 1993).

Tools are useful in error-prone tasks, such as logging issues in meetings, and in automating data collection, such as recording the time that inspectors have spent reading the document, calculating the number of defects, and generating reports from inspection data (Sembugamoorthy \& Brothers 1990, Votta 1993, Johnson 1998, Tyran \& George 2002).

Parts of the actual checking can be automated with tools. Anderson et al. (2003), for example, have implemented an analysis tool that can be used to discover defects in source code. One of the first inspection tools, ICICLE, included both support for static analysis of $\mathrm{C}$ code, and a platform for arranging electronic meetings (Sembugamoorthy \& Brothers 1990).

When interpreted broadly, tools can also mean other kinds of tools than software specifically dedicated to inspections. Because the $\mathrm{i} 3$ model allows the inspection process 
to be lightweight, the tools can also be light. For example, Wiegers (2002) provides an excellent set of forms and templates that can be used to gather defect-information and basic statistical data about review tasks. Gilb \& Graham (1993) also give comprehensive guidance on how and what data should be collected during inspections. These forms and templates are in paper form, but they can be easily transformed into simple spreadsheets and database applications that can be utilized to provide support necessary for inspectors.

Table 18 lists a few articles from literature dealing with computer support. Automatic defect-detection, computer-supported meetings and other types of inspection software have been a field of active research during the last ten years.

Table 18. Literature sources for the "Support with computer tools" activity.

\begin{tabular}{|c|c|}
\hline$\underline{\text { Reference }}$ & Notes \\
\hline (Anderson et al. 2003) & $\begin{array}{l}\text { Experiences with a static analysis tool are reported. The tool helps in } \\
\text { reducing the time required to inspect source code and is useable in formal } \\
\text { inspections. Integration and scalability of inspection tools are considered } \\
\text { important factors. }\end{array}$ \\
\hline $\begin{array}{l}\text { (Sembugamoorthy \& Brothers } \\
\text { 1990) }\end{array}$ & $\begin{array}{l}\text { Two typical features of tool-supported inspections combined in one tool: } \\
\text { meeting support and static analysis of source code. }\end{array}$ \\
\hline (Biffl \& Halling 2003a) & Tool support for data collection during inspections is necessary. \\
\hline (van Genuchten et al. 1997) & $\begin{array}{l}\text { Electronic meeting systems can help in arranging inspection meetings } \\
\text { virtually. }\end{array}$ \\
\hline (Votta 1993) & $\begin{array}{l}\text { Logging meetings can be replaced with computer tools to save inspectors' } \\
\text { time and resources. }\end{array}$ \\
\hline (Stein et al. 1997) & $\begin{array}{l}\text { The WWW provides a feasible framework for arranging place-independent, } \\
\text { asynchronous inspections. }\end{array}$ \\
\hline
\end{tabular}

Tool support for peer reviews is an interesting area of research. It is notable that there is still no single inspection tool that provides support for all types of reviews and all types of artefacts that are reviewed. This is because of the great variety in organizations' inspection cultures and process models, and in authoring tools and domains of development. However, there seems to be a fairly general agreement that suitable tools can facilitate the arrangement of inspections in challenging situations (such as distributed development) and improve the efficiency of the process by automating routine tasks.

\subsubsection{Maintain rules and checklists}

Individual checking is the most efficient part of the inspection process. The majority of the defects are found during the reading that an inspector does alone. Any support that the inspectors can receive at this phase is influential. The most concrete reading aid is an upto-date set of checklists, in which typical errors are listed. Use of the checklists can be tracked and analysed to keep checklists up-to-date. Errors that do not occur in the documents any more should be removed from the lists (Gilb \& Graham 1993).

Improved defect-detection techniques can improve the overall effectiveness of inspections. Techniques include simple checklists and more comprehensive reading 
techniques that are tailored for certain roles or system characteristics, such as a reading technique for inspecting object-oriented software (Porter et al. 1995, Laitenberger 2001, Dunsmore 2002).

According to Gilb and Graham (1993), checklists should be derived from coding standards and other established development rules used within the organization. Thus, a change to the rules causes modifications to the corresponding checklists. In order to be effective, checklists and other reading techniques must reflect to the standards currently in use.

Despite the name of this activity, it does not dictate that reading techniques other than ordinary checklists may not be used. The real meaning of this activity is that whatever the techniques are, they should be actively managed to ensure their appropriateness. If an organization is developing object-oriented software, their checklists, perspectives or scenarios should be defined with object-orientation in mind. As in the whole process, checklists should be tailored separately for each organization, and when they are established, they should be updated to improve the cost-effectiveness of the process.

In Table 19, literature references for this activity are summarized. Most of the articles dealing with checklists and other reading techniques concentrate on comparing different techniques, but there are a couple of experience reports that justify the importance of upto-date checklists on a more general level.

Table 19. Literature sources for the "Maintain rules and checklists" activity.

\begin{tabular}{ll}
\hline Reference & Notes \\
\hline (de Almeida et al. 2003) & A case of safety-critical software showing that checklists provide an \\
& excellent tool for revealing important and complex defects. \\
(Biffl \& Halling 2003a) & The article reports an experiment showing that reading techniques improve \\
& inspection effectiveness considerably. \\
(Dunsmore et al. 2003) & Checklists and other reading techniques for detecting errors in object- \\
& oriented code are presented. \\
(Gilb \& Graham 1993) & $\begin{array}{l}\text { An experience report from the Sema Group company, where the lack of } \\
\text { checklists and other guidelines for inspectors was one of the main concerns } \\
\text { after installing inspections. }\end{array}$ \\
A study in which defect data is fed back into the inspection process and used \\
to improve the process, and checklists in particular. \\
Defining perspectives for inspection requirements documentation provides \\
an effective defect-detection method, but only if an organization tailors \\
perspectives to its own needs and uses inspector feedback to continuously \\
refine the technique. \\
Checklists should not be too specific - that causes inspectors to focus on just \\
a few items and overlook the others.
\end{tabular}

Genericness of checklists is a recognized problem (Tervonen 1996). Even though checklist maintenance is strongly related to the "establish and improve the inspection process" activity, it deserves a separate activity status, as practically all inspection textbooks and a great deal of research reports on reading techniques emphasize that checklists are the cornerstone of detecting complex errors. 


\subsubsection{Refine information}

Data collected during inspections needs to be refined into reports, charts, and figures, to enable continuous monitoring and improvement of the process. Emphasizing benefits and measuring savings helps to keep management committed. Checklist usage, defectdetection rates, time required to inspect a document and analysis of defect sources are features that need to be investigated and communicated to management (Gilb \& Graham 1993, Wiegers 2002).

Data can serve the inspection team itself, as well (Weller 1993). Communicating the collected data back to the development team helps in decision-making and in understanding the dynamics of the development process. Making the results and benefits of inspections visible to all participants has a positive effect on the motivation of the team.

Gilb and Graham (1993) state that metrics are "the lifeblood of Inspection. They are key to its survival and productivity and must be totally integrated in the Inspection process on a daily basis." Once collected, metrics can be used to control the efficiency of inspections or to help in scheduling inspections (Gilb \& Graham 1993).

Table 20 gives examples of literature references emphasizing the significance of collecting and delivering inspection data. Measurements can be used to various purposes: the most obvious one is predicting the quality of the inspected artefact. With careful analysis, assumptions can be made about similar artefacts or the whole development process. Furthermore, the quality of the reviewing process itself can be evaluated with accurate data.

Table 20. Literature sources for the "Refine information" activity.

\begin{tabular}{ll}
\hline Reference & Notes \\
\hline (Briand et al. 2004) & $\begin{array}{l}\text { Data needs to be collected to support decision-making and the analysis of } \\
\text { inspection effectiveness. The article presents a model for understanding and } \\
\text { interpreting inspection data. } \\
\text { The article reports experiences of inspecting large amounts of code. A } \\
\text { special emphasis is placed on analysing and understanding the inspection } \\
\text { (Weller 1993) }\end{array}$ \\
$\begin{array}{l}\text { data in order to improve the development process. } \\
\text { The inspection process should be measured and data should be collected to }\end{array}$ \\
(Jalote \& Haragopal 1998) & $\begin{array}{l}\text { Two cases are presented showing that inspection data can be valuable in } \\
\text { demonstrating the benefits of inspections, in order to overcome installation } \\
\text { difficulties with the method. }\end{array}$ \\
(Chatzigeorgiou \& Antoniadis & $\begin{array}{l}\text { Inspection data should be used to estimate and control the time and effort } \\
\text { required when performing inspection tasks. With proper estimates, } \\
\text { 2003) }\end{array}$ \\
inspections can be coordinated within the project plan. \\
(George \& Janiszewski 2003)
\end{tabular}


Data-gathering is an inseparable part of peer reviews and almost every research article dealing with review methods involves data collection. In research, however, data is used mainly for comparing different methods or tools. There are, however, a few studies that contemplate exactly what data should be collected and how it can be used to upgrade the process.

\subsubsection{Check preconditions for inspection}

Checking the initial quality of the document to be inspected makes inspection more efficient. Gilb and Graham (1993) report a case in the Sema Group, where early experiences with inspections showed that the inspection leader has an important role in ensuring that the right documents are issued for inspections and the right inspectors are identified for the inspection. Ensuring that source documents are available is also one of the moderator's responsibilities before inspection (Gilb \& Graham 1993).

The overview phase is often underused. A well-organized overview should bring the inspectors to the same level of knowledge so that they can perform their reading tasks efficiently (Ackermann et al. 1989). Practically all review process models include a phase before the actual reading phases, in which the availability and maturity of the document is checked and the proficiency of inspectors ensured.

The artefact should be available and it should not contain trivial errors that can be found automatically or by the author, if she or he takes a second look at the document. Furthermore, enough skilled inspectors should be available and it should be ensured that they have adequate time to perform the review tasks. Unclear entry and exit criteria, lack of time, and concentration on trivial issues are all listed as problems in the case studies reported in (Gilb \& Graham 1993), for example.

In Table 21, some literature references justifying this activity are listed. The research mostly only briefly mentions experience reports of inspections that have failed because of careless utilization of entry criteria. Explicit studies of the effects of using entry criteria have not been reported. 
Table 21. Literature sources for the "Check preconditions for inspection" activity.

\begin{tabular}{ll}
\hline Reference & Notes \\
\hline (Kelly \& Shepard 2002) & $\begin{array}{l}\text { Code or documentation should be cleaned up for inspection to ensure that } \\
\text { time is not wasted on trivial issues. }\end{array}$ \\
(Blakely \& Boles 1991) & $\begin{array}{l}\text { The modules to be inspected should be selected according to their } \\
\text { complexity. Furthermore, modules that have not been modified in the } \\
\text { project should not be inspected. }\end{array}$ \\
& $\begin{array}{l}\text { If the quality of the input document for inspection is poor, it is hard to gain } \\
\text { positive results from inspections, and confidence in the whole process may } \\
\text { decrease. A skilled moderator is required to ensure the entry criteria. } \\
\text { The developers interviewed identify reviewer expertise and time and } \\
\text { resource issues as very influential on inspection results. } \\
\text { Researchers show that sampling techniques can be utilized before the main } \\
\text { inspection to evaluate the quality of documents to be inspected and to } \\
\text { identify the documents that most need inspection. }\end{array}$ \\
\end{tabular}

This is the first activity in the set of core activities that relate almost directly to different stages of inspection process models. The "check preconditions for inspection" activity combines the early stages, such as overview and kickoff (Gilb \& Graham 1993) or planning, scheduling and overview (Radice 2002).

\subsubsection{Plan the inspection}

Planning has been identified in all the most widely-known process models as the first phase of the inspection process after the decision to launch an inspection (Fagan 1976, Gilb \& Graham 1993, Wiegers 2002, Radice 2002). During the planning phase, team line-up, meeting times and other practical issues are addressed to ensure the smooth running of the review tasks.

The "plan the inspection" activity involves overall scheduling and goal-setting for the inspection iteration. Planning is needed to clarify what exactly will be inspected (Radice 2002) and when the inspection tasks are carried out (Gilb \& Graham 1993). Resource allocations and budgeting are needed for efficient project management (Radice 2002). Distributing all necessary material, including the document to be inspected, source documents and checklists, is also done during planning (Gilb \& Graham 1993).

Radice (2002) calls the first inspection stage "planning and scheduling", emphasizing the significance of an accurate timetable for the inspection tasks. Johnson and Tjahjono (1998) and Votta (1993) also give great weight to scheduling, due to the partly automated nature of the process. Another important purpose of planning is to clarify the procedure for the participants (Gilb \& Graham 1993, O’Neill 1997). Inspectors should be chosen carefully. Expertise and effort are required from the inspectors, in order for them to carry out their jobs effectively (Radice 2002).

Inspections should be planned alongside other project planning activities. Inspecting documents from the early phases of the development cycle is very profitable 
(Laitenberger 2001, Wiegers 2002) but can be easily omitted if inspections are not accurately planned in the project plan - before the development is in full swing.

The inspection plan must be ready as early as possible. This helps in securing the inspection-related tasks during the project. The inspection plan has to be coordinated together with the project plan in order to prevent a lack of inspection time and resources. Inspections of critical artefacts should not be treated as milestones, but as tasks, having duration and requiring resource allocations (Chatzigeorgiou \& Antoniadis 2003).

Table 22 summarizes some of the references that pay attention to this activity. Planning is identified as a crucial stage of the inspection process in most inspection studies and the importance of this activity has not been questioned in any studies.

Table 22. Literature sources for the "Plan the inspection" activity.

\begin{tabular}{ll}
\hline Reference & Notes \\
\hline (Berman 1998) & $\begin{array}{l}\text { Preparations such as ensuring the quality of the material, choosing the } \\
\text { correct checklists and briefing the participants are paid special attention. }\end{array}$ \\
(Ackermann et al. 1989) & $\begin{array}{l}\text { Planning is identified as the initial step of inspection. } \\
\text { Planning is critical, especially for ensuring that correct data is collected } \\
\text { (Barnard \& Price 1994) }\end{array}$ \\
during inspections. \\
An alternative inspection process without a joint meeting is studied. Proper \\
scheduling is crucial for meeting-less inspections. \\
(Parnas \& Weiss 1985) & $\begin{array}{l}\text { In the Active Design Review process, selection of inspectors and focusing } \\
\text { their efforts according to clearly-defined objectives is paid special attention. } \\
\text { Goals for the inspection need to be clearly defined. Furthermore, selection of } \\
\text { inspectors has to be done carefully according to the skills required by the } \\
\text { task. Ensuring that inspectors have adequate time to perform review tasks is } \\
\text { also important. }\end{array}$ \\
\hline
\end{tabular}

It is difficult to distinguish between planning and checking entry criteria in some review process models and experience reports. In the i3 model, these two activities are clearly separated, to stress the importance of both tasks: a document that is not mature enough to be inspected should not enter the inspection process, and when a document is ready for inspection, the process should be carried out in a manageable and efficient way.

\subsubsection{Find issues in the artefact}

Finding possible errors in the document under inspection is, of course, the heart of the inspection process. The document is typically read through individually by each reviewer. Fagan (1976) calls this phase preparation and Gilb and Graham (1993) individual checking. Doing this phase properly is crucial to getting the most out of inspections (Biffl \& Halling 2003a, Votta 1993, Gilb \& Graham 1993). In addition to individually-done reading, defects can be searched for during inspection meetings, in which all team members are present.

Several studies have investigated both the individual checking and inspection meeting phases of the process. Optimal rates for reading, defect-detection and logging have been 
sought and a general agreement is that reviewing should not be done hastily. The amount of inspected material should also be manageable. During meetings, discussion should be controlled to keep the logging rate optimal (Gilb \& Graham 1993, Wiegers 2002).

The right people have to participate. Even though a few inspectors can be chosen to the inspection team to practice and learn, most of the participants should be capable of finding defects. Sometimes this may be difficult. For example, in a requirements specification inspection, it may be helpful to have a customer representative present (Wiegers 2002). Gilb and Graham (1993) also report a case study showing that using outside reviewers can be beneficial.

Table 23 lists examples of references that demonstrate different aspects of the defectdetection activity. Typically, most of the inspection research is carried out in this area. There are comparative studies of reading techniques, studies investigating the necessity of meetings, and studies on the effects of inspector expertise on inspection results.

Table 23. Literature sources for the "Find issues in the artefact" activity.

\begin{tabular}{|c|c|}
\hline Reference & Notes \\
\hline (Parnas \& Weiss 1985) & $\begin{array}{l}\text { The Active Design Review process makes defect-detection effective by } \\
\text { adjusting inspector skills according to the document in question. } \\
\text { Furthermore, interaction between developers and inspectors is encouraged, } \\
\text { in order to focus on important issues. }\end{array}$ \\
\hline (Petersson 2001) & $\begin{array}{l}\text { Empirical inspection data is analysed to demonstrate the impact of an } \\
\text { individual inspector on the team's effectiveness. The more skilled the } \\
\text { inspectors are, the smaller the inspection team can be to achieve meaningful } \\
\text { results. }\end{array}$ \\
\hline (Porter et al. 1995) & $\begin{array}{l}\text { Ad hoc, checklist-based and scenario-based defect-detection techniques are } \\
\text { compared in this article. Systematic reading techniques help in focusing on } \\
\text { the most important issues, and improve the performance of individual } \\
\text { inspectors. }\end{array}$ \\
\hline (Porter et al. 1998) & $\begin{array}{l}\text { The nature of the code under inspection, and of reviewers and authors have a } \\
\text { considerable impact on inspection performance, suggesting that providing } \\
\text { support for the defect-detection task helps in improving the effectiveness of } \\
\text { the inspection. }\end{array}$ \\
\hline (Stein et al. 1999) & $\begin{array}{l}\text { Understanding annotations made to an artefact requires that related } \\
\text { information is understood (artefacts produced in different development } \\
\text { phases, or different versions of the artefact, for example). Providing such } \\
\text { information for inspectors may help them to carry out inspections more } \\
\text { efficiently. }\end{array}$ \\
\hline (ANSI/IEEE 1989) & $\begin{array}{l}\text { Inspectors should be provided with standards and previously inspected } \\
\text { documents from the same domain to ensure they have all possible } \\
\text { information about the system to be built. }\end{array}$ \\
\hline
\end{tabular}

Different aspects of defect detection have drawn much research attention away from the other important inspection tasks. The defect-detection phase is a convenient target for quantitative studies, as it is easy to collect basic metrics concerning defect types and 
inspector performance. However, other influential factors, such as ensuring an adequate level of training or domain knowledge of the inspectors, are often overlooked.

\subsubsection{Categorize defects}

Categorizing defects means that each suspected item in the reviewed document is explored in order to identify how serious it is and why it has occurred. According to Radice (2002), identifying classes, types and instances of defects helps to prevent their recurring in the future, and to find their probable causes in the environment.

Shirey (1992) points out that long-term benefits are gained from inspections when process errors that generate faults can be identified. This requires causal analysis and defect-prevention actions, which are enabled by decent defect categorization.

Defects can be classified on numerous different bases. Typically, the initial severity, corresponding checklist item, and possible origin of a defect are recorded by the inspector. The author makes the final decision on how a defect is classified (Gilb \& Graham 1993). Information on where the defect was caused and where it was found can also be collected during the review (Radice 2002).

There should not be too many classification principles and categories. Two-class severity classification (major/minor) may be adequate, even though most organizations utilize three or more severity classes. For example, (Doolan 1992) introduces a minor/major/super major classification, and (Kelly et al. 1992) biases the classes in the opposite direction by using major/minor/trivial classes.

Classification can also be done on a basis other than severity. If the defect originates from a mistake in previous development phases, source documents can be used to track its root cause. Furthermore, classification can be based on perspectives, scenarios or checklists that the inspector has used. For example, a defect may concern a user interface or database.

Table 24 lists literature references that address defect classification. Defect data has been used to analyse the inspection process from a variety of perspectives. Typically the data helps in resolving the root causes of certain types of defects and eliminating these shortcomings in development. Categorization also enables efficient analysis of the inspection process and ensures that inspections uncover serious defects instead of trivial ones. 
Table 24. Literature sources for the "Categorize defects" activity.

\begin{tabular}{ll}
\hline Reference & Notes \\
\hline (Porter \& Votta 1997) & $\begin{array}{l}\text { The article states that providing prescription documents for organizing the } \\
\text { inspection data is essential for successful inspections. }\end{array}$ \\
(Shirey 1992) & $\begin{array}{l}\text { Without categorization of the revealed defects, their future prevention is not } \\
\text { possible. }\end{array}$ \\
Categorizing findings according to the development phase enables effective \\
root cause analysis of the defects. \\
(Chaar et al. 1993) & $\begin{array}{l}\text { A study demonstrating evaluation of software inspection effectiveness with } \\
\text { the aid of comprehensive defect classification. }\end{array}$ \\
(Chernak 1996) & A research article applying Orthogonal Defect Classification (ODC) to \\
& enable causal analysis of defects discovered during code inspections.
\end{tabular}

This activity emphasizes the value of proper inspection data collection. Without data, improving the product or process quality will be based on presumptions. To utilize the full potential of the method, inspectors need to not only point out the possible flaws in artefacts, but also think carefully about their real meaning and grounds.

\subsubsection{Make corrections}

This activity has been little discussed in the inspection literature. In the original Fagan method, the defect correction activity is called "rework". Fagan simply states that during this phase "all errors or problems in the inspection report are resolved by the designer or coder/implementor" (Fagan 1976). The author is also given authority to accept or reject findings. The author is then expected to write down rationales for his or her decisions (Gilb \& Graham 1993).

Radice (2002) states clearly: when you find a defect, fix it. Shirey (1992) lists the most common reasons for not fixing defects discovered in inspections as follows: 1) the inspection data was lost, 2) the author didn't understand the inspection data and 3) there wasn't time in the schedule.

In Table 25, a couple of references emphasizing the importance of this activity are listed. There are few articles which deal explicitly with the rework phase. Obviously, ignoring inspectors' findings makes the whole inspection fail.

Table 25. Literature sources for the "Make corrections" activity.

\begin{tabular}{ll}
\hline Reference & Notes \\
\hline (Gilb \& Graham 1993) & $\begin{array}{l}\text { Points out a problem that occurs if the defect is found in a source document: } \\
\text { who is responsible for fixing it and what other changes will it cause? }\end{array}$ \\
(Shirey 1992) & $\begin{array}{l}\text { Ignoring the inspection results makes the whole process fail. Defects } \\
\text { uncovered have to be acted on. } \\
\text { (Doolan 1992) }\end{array}$ \\
& $\begin{array}{l}\text { The moderator has to check that the author has taken an action for each } \\
\text { deted defect. }\end{array}$ \\
\hline
\end{tabular}


This activity corresponds to the rework phase, which is defined in all major inspection process models. This phase - in particular the implications that an inspection may have on other documents and on the development process - has not been particularly well studied in the inspection literature.

\subsubsection{Conclude the inspection}

The ultimate goal of inspections is to reduce the number of predicted major defects remaining in the document to a tolerable and calculable level. The exit criteria define as clearly as possible the point at which it is appropriate to use the inspected document as a source in a downstream process. When an artefact meets the exit criteria, it also will have a known quality status.

In addition to ensuring that the document has passed an inspection, a firm conclusion is needed for the inspection to ensure that the inspection process itself has achieved an adequate quality level. In order to evaluate the inspection process quality, the number of defects that inspection has uncovered needs to be estimated, as well as the number of defects that remain in the document.

For example, Galin (2004) emphasizes the importance of post-review activities that distinguish inspections from other peer review methods, in particular less formal walkthroughs. The inspection does not end with a review session. Instead, it is important that corrections are made to the inspected document, and inspection data is reported in appropriate directions in order to initiate process and productivity improvement.

There are methods for supporting the decision whether inspection should stop or whether it should continue. Estimating the number of remaining defects in inspected documents is important when making the decision. For example, (Briand et al. 2000) reports experiences on capture-recapture models for predicting the number of remaining defects.

Table 26 summarizes some of the literature references for this activity. The research on the final phases of an inspection cycle concentrates on providing methods and support for making a decision on re-inspection and estimating the number of defects remaining in the document. 
Table 26. Literature sources for the "Conclude the inspection" activity.

\begin{tabular}{ll}
\hline Reference & Notes \\
\hline (Briand et al. 2004) & This study presents a model for understanding and interpreting inspection \\
& data to support decision-making on whether an inspection should be stopped \\
& or the document should be re-inspected. \\
& The researchers point out that concretely defined exit criteria are often \\
& missing from companies' inspection processes, and present encouraging \\
(Yin et al. 2004) & results about the use of self-assessment to determine need for re-inspection \\
& or exit decision. \\
& This article reports an experiment in which subjective estimations are used \\
& to ensure that the density of remaining defects is below a predefined level, \\
and that the inspection process has achieved at least a minimum level of \\
effectiveness. \\
Follow-up is a crucial part of a successful software inspection process. \\
A case from the Sema Group, where clear entry and exit criteria are \\
considered to be important success factors of recently installed inspections. \\
\hline
\end{tabular}

A clear ending to an inspection iteration is necessary, to release resources assigned to it and to enable the transfer of the artefact into subsequent development phases. After rework, the document can be frozen and the inspection data analysed.

\subsubsection{Summary}

The activities defined in the $\mathrm{i} 3$ model do not directly correspond to traditional phases in any inspection process model. There are similarities, especially in the core activities, but there is also a notable difference from conventional inspection processes: activities in the i3 model describe the tasks that need to be carried out to complete an inspection, but the model allows varying implementation of these tasks. For example, removing the inspection meeting and replacing it with tools changes the process significantly. The previously presented inspection process models are built on the assumption that defined steps are performed in a predefined manner. In this respect, the i3 model can be called a reference model for the inspection process, and its actual realization is left for organizations to implement.

The i3 model is needed to enable evaluation of the inspection status within an organization. Thus, the i3 model is a capability model for inspections. Assessing the performance of review tasks without an underlying model would be subjective and arbitrary. However, using the model to benchmark or compare organizations is not possible because activities can be implemented differently in different contexts, as mentioned.

Rationales for the activities are extracted from literature, including textbooks and research articles regarding inspections and peer reviews. During the last three decades, these methods have been a subject of active research. It is not feasible to list all the papers that concern inspections here. Instead, the fundamental elements of the inspection method and interesting variations of its procedures are summarized. 


\subsubsection{Indicators}

An indicator is a work product that is produced during the software development process and that is in some way related to the inspection process. Each indicator is associated with a set of activities, and the same indicator can different meanings and significance in distinct activities.

There are two types of indicators, enabling indicators and verifying indicators. Enabling indicators make the inspection process possible. They provide the means to carry out specific inspection activities, and they can be seen as mandatory preconditions for the activities. Another interpretation of an enabling indicator is that it is an input for a certain inspection activity. The absence of an enabling indicator strongly suggests that the corresponding activity does not exist or exists only partially. Verifying indicators are work products that are composed during certain inspection activities. Thus, they are outputs of activities, and provide evidence that an activity has produced appropriate and adequate results. Even though a verifying indicator can be found, it does not guarantee that the corresponding activity exists, as the work product may have been produced in a phase of development other than inspection.

Thus, indicators are traces which suggest that certain inspection-related tasks have been carried out during the development. This evidence can be tracked down most clearly if they exist in documentation. In many cases, however, there is no written material to prove the existence of an indicator. For example, particular people in the company may always have certain viewpoints in the inspections, based on their everyday responsibilities. Database designers, for example, may always inspect documents from the database perspective. This implicit role assignment is usually adequate in a very small company and there is no particular need for written role descriptions, which is one of the indicators. For that reason, an interview is the best technique to discover whether certain indicators exist in the process. Furthermore, the scope and the adequacy of detail of the indicators has to be evaluated carefully case by case.

There are 29 indicators in the i3 model. These indicators are listed in Table 27. They have been selected by analysing several inspection process variations. Recognizing the most vital sub-processes and their inputs and outputs - such as open issue log produced in the logging meeting phase in Gilb \& Graham's inspection model (Gilb \& Graham 1993) - provides the basis for the indicator list. 
Table 27. The i3 indicator list.

\begin{tabular}{llll}
\hline \multicolumn{2}{l}{$\begin{array}{ll}\text { Indicator } \\
\text { number }\end{array}$} & Indicator name & \multicolumn{2}{l}{ number } & Indicator name \\
\hline 1 & Entry decision & 16 & Collaborative tools \\
2 & Entry criteria & 17 & Data gathering and analysis tools \\
3 & Schedule & 18 & Rules \\
4 & Inspection resource allocation & 19 & Role descriptions \\
5 & Issue list & 20 & Checklists \\
6 & Categorization & 21 & Pool of inspectors \\
7 & List of corrections & 22 & Inspection leader selection \\
8 & Acceptance of corrections & 23 & Request for training \\
9 & Request for rework & 24 & Quality resource allocation \\
10 & Request for rework on another artefact & 25 & Process data measured \\
11 & Exit decision & 26 & Reports \\
12 & Exit criteria & 27 & Improvement suggestions \\
13 & Forms & 28 & Process change requests \\
14 & Tutorials & 29 & Inspection process definition \\
15 & Courses & & \\
\hline
\end{tabular}

The indicators are ordered. This order is based on experiences obtained during assessment sessions: items that are inter-related are close to each other on the list. The interview proceeds more naturally and efficiently when one can concentrate on one subject at a time. Furthermore, some items in the indicator list can be assessed simultaneously. The first two (entry decision and entry criteria), for example, complement each other and it may be practical to handle them together.

The matrix in Figure 25 shows the relationships between indicators and activities. A letter $\mathrm{V}$ means that a specific indicator is a verifying indicator for an activity. For example, the entry decision indicator is a verifying indicator for activity A.7, "check preconditions for inspection". A letter E means that the indicator is an enabling indicator for an activity. For example, the same indicator (entry decision) enables activity A.9, "find issues in the artefact". The same indicator can have both enabling and verifying roles in certain activities. 


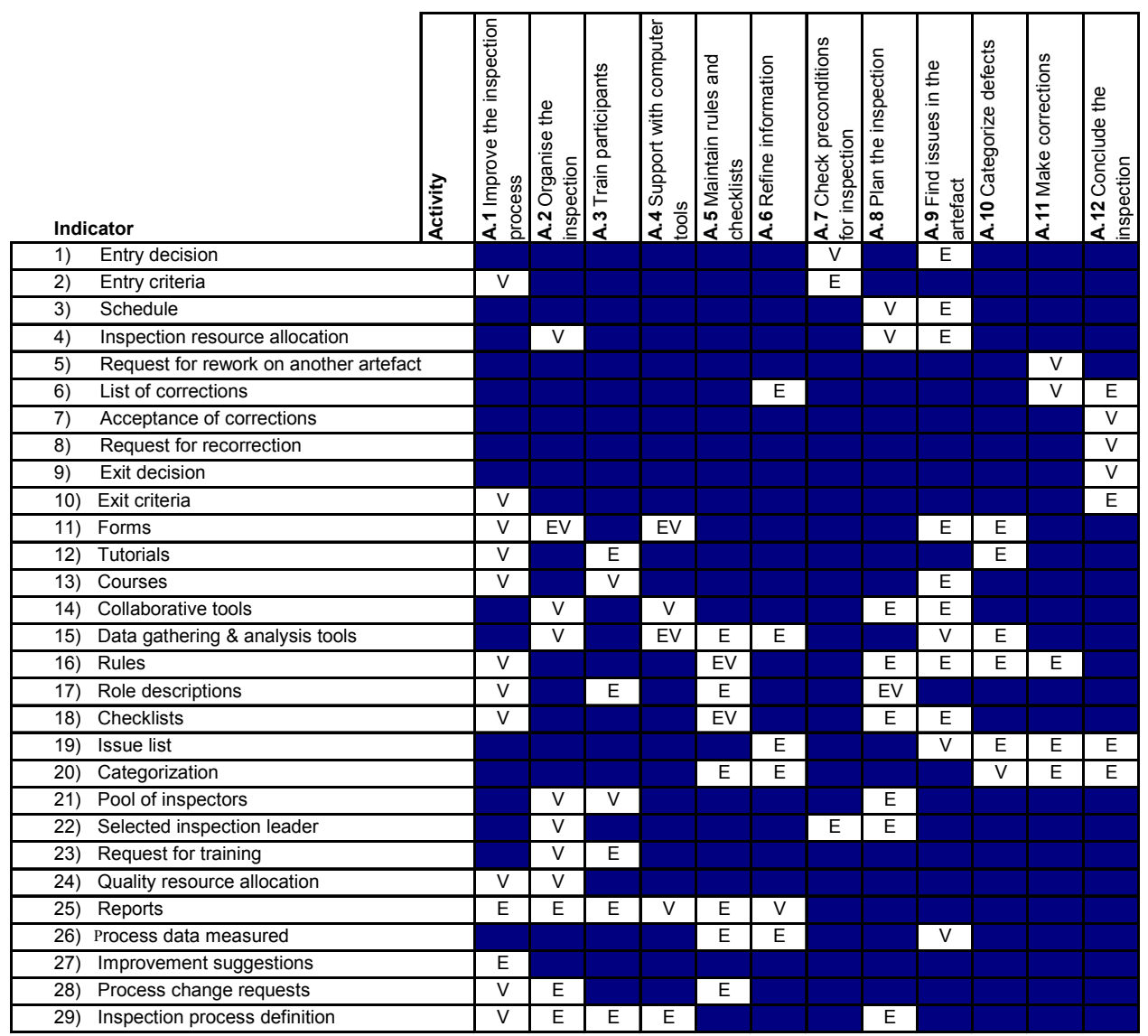

Fig. 25. Indicators mapped to activities.

In the following, the indicators are described in more detail. As the number of indicators is quite large, they are grouped by the type of activity that they are mainly connected to. Even though indicators are grouped by activities, they can also have connections with other activity types. For example, checklists have different roles in an organizational context (they need to be managed) and in core activities (they are used to detect defects). A couple of examples are also provided for each indicator to exemplify their implementations in a real environment.

\subsubsection{Indicators related to organizational activities}

Entry decision. Each inspection has to be preceded by a clear resolution point, in which a judgment is made concerning whether the inspection should start or not. The purpose of such a decision is to ensure that incomplete artefacts will not get into the inspection process. The lower the quality of the material to be inspected, the lower the results of the 
inspection. It is not reasonable to sacrifice inspectors' resources in reading through a document that will most probably fail the inspection. It is usually the responsibility of the inspection leader to check that certain preconditions are met. A project manager can also make the decision. The decision should be based on precise entry criteria.

The entry decision indicator can take varying forms in different projects and companies. Examples of the entry decision indicator include:

- An e-mail notification to the participants that states whether there is justification to start the inspection.

- A "request for inspection" form approved by a moderator.

The entry decision is separated from entry criteria to identify if the inspection is managed also in reality. If there is concrete evidence of an entry judgment having been made, it indicates that each inspection iteration is planned and has a leader.

Entry criteria. The entry criteria are used by the inspection leader who makes the entry decision. The criteria should be formal and written in the quality handbook or other software development guide used within the organization. The criteria have to be practicable and they have to address different types of artefacts adequately. The prerequisites for a requirements specification inspection are totally different from the prerequisites for a code review, for example.

Examples of different types of entry criteria indicator include:

- The inspection leader's checklist for inspection preconditions. An example checklist entry could be "Does every method in the source code have adequate comments?"

- A tool that checks that a document has correct values in the author, version history and other similar fields.

The level of formality of the entry criteria indicator depends heavily on the organization. In a very small company, it does not necessarily make sense to define different entry criteria for each application domain, if the criteria would be used only once. In a larger organization that has separate divisions, each developing a certain type of software, it could be feasible to define entry criteria for each domain.

Schedule. Each phase of the inspection should have a predefined schedule. The schedule is especially important, because there are usually several steps in the process and a few people participating. A minor delay at the beginning of the process may accumulate along the way and finally result in serious time-loss in the project. The schedule has to be planned, written down and communicated to the participants. The progress of the inspection tasks and the implementation of the schedule have to be monitored.

Examples of the schedule indicator include:

- A tool that sends reminders to the inspectors about their deadlines for certain tasks.

- An e-mail message including the document to be inspected and a notification of the logging meeting date.

The schedule indicator is perhaps the most obvious evidence of inspection planning. Furthermore, schedule is one of those indicators that should always exist in some written form. Without scheduling, reviewing is ad hoc, not fulfilling the measurability and formality characteristics of inspections. 
Inspection resource allocation. Depending on the comprehensiveness of the defined process, review tasks require a certain amount of time from the participants. The required effort should be estimated in the project plan to ensure that people will have the motivation and ability to carry out the inspection. Furthermore, inspectors have to be officially invited to participate in an inspection. The allocation of resources has to be documented in order to avoid misconceptions.

In addition to human resources, inspection tasks may involve computer and software tools, meeting rooms and equipment, and other facilities, which have to be available during specific phases of the inspection process. All significant resource demands have to be described in an inspection master plan.

Examples of the inspection resource allocation indicator could include:

- A project plan including "inspection" as a part of every document-producing task.

- An inspection master plan including the reservation of a conference room for the logging meeting.

Adequate resourcing is one of the cornerstones of the inspection process. The time that is needed to carry out review tasks should be written down in development plans. Otherwise inspections are considered not as part of the company's quality policy, but as an incidental activity.

Exit decision. The last two pieces of evidence indicating that reviewing has been performed satisfactorily are related to the conclusion of the inspection. As with the entry decision, an unambiguous resolution point is needed to conclude the inspection.

The purpose of the exit decision is to confirm that the inspected document is of adequate quality and is allowed to be used in the development. If there are any uncertainties concerning the status of reviewed artefacts, the artefacts can be interpreted in different ways by different engineers, possibly causing new defects and the need for extra discussions during the development.

Checking that the postconditions are met is usually the inspection leader's responsibility. The decision should be based on precise exit criteria.

Examples of the exit decision indicator include:

- A closure statement in the inspection meeting minutes.

- A document status change in the version control system, freezing the document and making it available to developers.

As with the entry decision and entry criteria indicators, an exit decision and exit criteria are needed to emphasize the controlled nature of the process.

Exit criteria. Exit criteria are contained in a document or tool that guides the inspection leader's judgment of whether the inspection should be concluded or not. This guidance can be printed in the quality handbook of the organization, or in the inspection process description, for example. The criteria should be different for different types of documents. The criteria have to be clearly identifiable and quantifiable. For example, they may concern the number of major defects per page found in the document, or an estimate of the number of remaining defects.

Examples of the exit criteria indicator include: 
- A set of metrics identified in the quality handbook.

- The inspection leader's private checklist for document maturity.

As with entry criteria, the formality of this indicator depends on the number of inspections, inspection leaders, projects and different types of domains that the company deals with.

Pool of inspectors. The pool of inspectors is a typical resource pool. It is taken advantage of when planning projects and inspections. The list of available inspectors should include information about the special expertise of each person. The list should preferably contain only people that are qualified for their roles as inspectors or inspection leaders.

Resource pools are needed for basic project resource allocation, thus the pool of inspectors is not necessarily an inspection-specific repository. It may be an enhancement to ordinary personnel management routines and tools.

Examples of this indicator could include:

- An inspection-specific expertise statement for a person in a human resources management system.

- A paper-form list of people with inspection experience, maintained by the inspection leader.

This indicator stresses the manageability and orderliness of the inspection process. The indicator relates closely to scheduling and resource allocation, with the aim that review tasks should be made visible in the development cycle.

Quality resource allocation. Because systematic inspections require substantial investment, the organization should strive to continuously control the effectiveness of the process and improve it when possible. The inspection process as a whole has to have ownership defined, and be maintained as necessary. The quality resource allocation indicator emphasizes process ownership. The quality assurance group in the organization is typically responsible for keeping the inspection process up-to-date. In order to achieve this, the software quality assurance function needs resources in order to respond to the training and other needs of the inspection process.

Examples of this indicator include:

- The quality handbook defines the ownership of the inspection process.

- The job description of the quality manager includes the maintenance of the inspection process.

The existence of this indicator shows that the company is committed to the inspection process at an organizational level, bringing continuity to inspections.

Improvement suggestions. The improvement suggestions indicator concerns improvement ideas that are presented for the inspection process or the development process as a whole, not suggestions towards solving problems in the inspected artefact revealed during inspections. In addition to measured data on the process, it is useful to collect informal ideas that inspectors and inspection leaders have come up with during the inspection. 
- Improvement suggestions collected after an inspection This is referred as "third hour" by Gilb and Graham (1993).

- A feedback form for inspectors to report shortcomings in the process.

For example, the inspection process introduced by Gilb and Graham (1993) includes an optional phase for collecting proposals for enhancement of the process. These issues should not be discussed during the inspection itself, so the list of improvement suggestions should not be included in the list of defects.

Inspection process definition. The process definition should be a clear, companyspecific description of the stages and tasks that are carried out during one inspection iteration. Particularly in small companies, the process needs to be adapted. For example, a small company usually does not have a separate quality assurance team to guide the installation of the inspection. The advantage of this is that knowledge sharing is immediate, but at the same time there is a danger of serious personality conflicts, as the role of individuals is emphasized in small teams (Gilb \& Graham 1993).

Examples of this indicator include:

- A quality handbook that describes the course of an inspection. The phases, participants and outcomes of the process are listed.

- A reference to a universal inspection model (such as Fagan's inspection) that is utilized directly in the organization.

This indicator is intentionally last in the list that is gone through during the evaluation interview. Throughout the evaluation phase, the numerous aspects of the inspection process are discussed, and the interviewees gain a more comprehensive understanding of the inspection process as a whole, making it easy to assess whether the process definition in use takes into consideration all the required aspects.

Request for training. Typically a human resource management function is responsible for arranging adequate training to ensure that there is a sufficient number of inspectors and inspection leader available within the organization at any time. The request for training indicator can be any type of evidence showing that the need to improve the skills of the people participating in the process has been anticipated - and that the process owners react to these needs. Training is usually requested by inspectors, inspection leaders or project managers.

Examples include:

- An informal e-mail message stressing that inspectors need training.

- A questionnaire to reveal the misunderstood or obscure elements of the process, completed after each inspection.

This indicator relates to the organizational ownership and management of the process. Training may be requested by an individual who has not previously carried out inspections, for example. 


\subsubsection{Indicators related to the core activities}

Issue list. The issue list is perhaps the most obvious indicator in the $\mathrm{i} 3$ model. It is the list of defects that is combined from the findings of each inspector. It is the most essential outcome of an inspection iteration. If possible defects are not systematically logged, it is hard or impossible for the author to modify the inspected document.

Examples of the issue list include:

- An e-mail message from the inspection leader to the author including items found during inspection.

- A database including locations, checklist references and comments about dubious items.

The actual form of the issue list may vary - it can be unstructured or strictly formatted, composed with a comprehensive logging tool or simply hand-written. Without an issue list, however, there is no evidence that the inspection has been carried out at all. Thus, the issue list acts as an enabling indicator in quite a few activities.

Categorization. Different inspection process models offer slightly different approaches for defect categorization. All stress that at least the severity of a defect has to be recorded. The categorization is usually done using a three- to five-level scale (e.g., major, minor, cosmetic). In addition to severity classification, inspectors are often asked to classify defects according to defect type and source.

It is necessary for defect categorization to be based on an explicitly defined set of classification rules - checklists, severity rankings, defect-origin analysis and so on. Thus, this indicator is also related to checklists and rules. Without these a comprehensive categorization is not possible.

Examples of the categorization indicator include the following:

- Categorization is integrated into the issue list.

- A logging tool provides a form for defect classification, including predefined options for severity and checklist reference.

Categorization is separated from the issue list indicator to emphasize its significance. Separation helps the assessors to evaluate the quality of the inspection from different viewpoints. The existence of an issue list proves that the inspection has been held, and furthermore, if items in the list have been categorized, it will become possible to measure the effectiveness of the process.

List of corrections. List of corrections is a document in which the author has recorded his or her rework actions. The author can log corrections straight into the issue list, or there may be a separate document for information about modifications. This indicator reveals whether the issues found are really addressed by the author, and at the same time records judgments made by the author about whether certain issues are defects or not, with reasons. Gilb and Graham (1993) in particular emphasize the author's role in making final decisions on defects and commenting on the corrections.

Examples: 
- The issue list is transferred into a to-do list in an inspection tool, in which the author stores his remarks after the inspected document has been updated.

- The author sends an e-mail to the inspection leader, listing all modifications made to the document.

Rework and follow-up are perhaps the most overlooked phases of the inspection. The author has a vast responsibility in correcting the artefact. If he or she does not understand the issues reported or makes an inaccurate judgment on an issue, inspection efforts are lost.

Acceptance of corrections. The inspection leader has to check that the author has interpreted the issue list or to-do list correctly and made adequate corrections. The author can also communicate with the inspection leader during the rework phase, which also indicates that the author has received approval for modifications.

Examples of the acceptance of corrections indicator include:

- The inspection leader carries out a cursory check of the updated document and changes its status to "approved".

- The inspection leader closes tasks in the inspection rework to-do list after modifications have been made.

This indicator has been introduced to emphasize the controlled termination of an inspection and ensure that full advantage is taken of the results. The level of formality of this indicator can vary greatly between organizations.

Request for re-correction. Request for re-correction indicates that all corrections made by the author are not automatically approved. There may be dependencies between modules or documents, resulting in a situation where a modification to one document may necessitate modifications to the other. Such interface linkages are often hard to notice. This indicator is strongly related to the acceptance of corrections and these are usually evaluated jointly.

Examples include:

- The author submits the updated document to the inspection leader, who performs a cursory check of it and points out some deficiencies.

- Correcting a defect has caused a change in the module interface, affecting all dependent modules. Backwards compatibility needs to be ensured with additional corrections.

As with the acceptance of corrections indicator, this indicator stresses controlled inspection closure. In the same way, this indicator can take either a strict or an informal form.

Request for rework on another artefact. It is possible that a defect may have influences on other artefacts besides the one under inspection. In particular, change requests to component interfaces during the implementation phase can lead to significant modifications to related components. Similarly, changes to outside artefacts are required if major deficiencies are found in the software design when coding has already started.

Examples of this indicator could include the following: 
- The implementation language does not support features that have been used in the design. The design needs updating.

- An external component has to be updated to provide a compatible interface for the current implementation.

This indicator seldom exists as an inspection-specific form or template. The change request usually takes a form similar to an ordinary change request, and it is preferably stored in the bug tracking system. This indicator is separated from the request for recorrection indicator to emphasize the need for categorization and analysis of the root causes of defects.

Inspection leader selection. Selection of the inspection leader indicates that the inspection is planned. In smaller companies or departments there is perhaps only one person who is responsible for inspections. In such cases, the selection is obvious and implicit. Whenever possible, the leader ought to be chosen from the inspection resource pool. The leader has to be qualified for the moderator task and the selection has to be done in a controlled manner before starting the inspection. The leader will preferably have previous experience of enacting inspections. The inspection leader is also often called a moderator.

Examples of this activity include:

- The leader selection shows in inspection invitations.

- The leader name is identified in an inspection master plan.

Inspection leader selection is listed in the indicators related to the core activities, as the role of the leader is the most concrete evidence of inspection planning, which is one of the core activities. The other core activities are also controlled by the leader.

\subsubsection{Indicators related to the supporting activities}

Forms. Inspections involve a great deal of data, which can be gathered either manually or automatically. Whichever gathering method is employed, the content and form of the data has to be explicitly defined. The quality handbook or process description has to include templates to describe what information is collected during inspections and how it is represented to different interest groups.

Data gathering is crucial for sufficient analysis of the causes of defects. Furthermore, without data the improvement of the inspection process would not be possible. Thus, providing adequate means for the data to be gathered is very important. Forms and templates are concrete enablers for this. Without any tools it is not likely that inspectors would be able to report their findings coherently and consistently.

There should be forms and templates for each significant phase of the inspection, such as meeting minutes, report of individual inspection and summary report. In addition to the actual data, each form should include certain administrative fields, for example date, name and meaning of the document, and instructions for its usage.

Examples of the forms indicator include the following: 
- Copies of universal inspection forms, such as the ones in (Wiegers 2002).

- Spreadsheet templates for reporting issues, with formulas for calculating simple metrics.

This indicator is separated from the actual data indicator to stress that the process needs to be supported by adequate tools. Whether the forms are in print or computerized is not important, as long as they exist and are available for the inspectors.

Tutorials. There are two indicators that directly concern inspection process training: tutorials and courses. Tutorials are written instructions for the performance of inspection tasks and they provide guidance on carrying out certain inspection procedures or using the tools. They can be in either paper or electronic form. Tutorials are self-access study materials, in which inspectors' routine is strengthened by examples and exercises of the review procedures.

Examples of this activity include:

- An inspection tool with integrated online help and demonstrations.

- A quality handbook that is accessible to inspectors and that includes detailed information on how to perform review tasks.

Individual tutorials are insufficient for the training of new inspectors, and a proper installation of the inspection process involves full-scale courses, as well.

Courses. Courses can be arranged within the organization, but in many cases outside expertise is taken advantage of, especially if the company has started inspections recently. This indicator requires that inspectors and inspection leaders have received a certificate to show their qualification. The courses indicator is separated from self-study material tutorials, that is - to emphasize that, on occasion, some type of audit is needed to ensure the validity of the process and keep people informed about possible updates to the process. Furthermore, newcomers should be familiarized with inspections, and the most effective method for that is proper training, including intensive courses.

In an ideal situation, all participants in inspections ought to hold certificates. A certificate ascertains that a person has knowledge of the inspection process in general, and of the particular review method used in the organization. On the other hand, courses that are provided for the personnel should encompass both general and company-specific aspects.

Examples of this activity include:

- A course provided by an outside consultant.

- A course provided by the quality assurance group of the organization.

Episodic courses are insufficient for continuous maintenance of inspection expertise, and a proper installation of the inspection process involves self-study material, referred to as "tutorials" in the i3 model.

Collaborative tools. Different types of tools are needed to support review tasks. In addition to analytical tools, inspections require a great deal of communication and workflow management. There are many routine tasks, such as material handling, scheduling, and the recording of meeting minutes. Many of these error-prone tasks can be automated to assist the inspection leader. 
Tools can be tailored for the inspection process specifically, or they can be generic software: electronic meeting support systems, video conferencing systems, workflow management systems or even basic e-mail. The correct combination of software tools depends on the essential needs of the company. If inspections are common practice, the utilization of inspection-specific tools may be feasible.

Examples that show the existence of the collaborative tools indicator include:

- Use of e-mail to distribute material and report defects from the individual preparation phase.

- An instant messenger to discuss suspect items in the document with other inspectors.

Collaboration tools can be integrated with development tools or with analytical inspection tools. For example, modern word-processing software has online annotation features.

Data gathering and analysis tools. Collecting and analysing statistical data is an important part of the inspection process. With concrete, measured numbers, it is possible to determine optimal reading rates, set realistic schedules, and evaluate the quality of the reviewed document. Furthermore, gaining and maintaining management commitment is much easier with facts and figures.

Examples of data gathering and analysis tools include the following:

- The document under inspection is placed on a server and annotations can be made directly to the document via a network.

- A spreadsheet application is used to report defects and visualize defect densities, severities and types.

This indicator is vital for the efficient refinement, processing and reporting of benefits and bottlenecks in the inspection process. As with the other inspection tools, this indicator can be implemented with greatly varying levels of automation.

Rules. This is an indicator that is not directly connected to the inspection tasks. However, many parts of the inspection process require precise rules to be defined before the inspection can succeed. The development has to be disciplined, and it further regulates how the inspection process is built on it.

This indicator refers to methodological heuristics as well as style guides for a variety of artefacts. For example, heuristics can be design instructions for object-oriented development, and style guides may include documentation rules for class diagrams or project plans.

Examples include the following:

- Coding conventions for a specific programming language.

- A template for a requirements document.

Standards and rules for development form the basis for the detection and classification of defects, as the main idea of the inspection process is to reveal violations that an artefact contains when compared to these standards and rules.

Role descriptions. Roles are defined in the quality handbook or similar document. The use of roles aims at making inspections more effective. When each inspector is given a role, several viewpoints can be attained on the same artefacts. This should reveal more defects and remove duplicate findings. 
Roles have to be relevant to the organization. Roles can be derived from the underlying development method, or they can be based on the functional break-down of the system. For example, one can read through an artefact from the maintainer's point of view, or focus on database interfaces. The role descriptions indicator can be interpreted to include perspectives and other methods that are aimed at focusing the inspector's reading.

Examples include:

- A checklist concentrating on the issues that a customer would pay attention to.

- A scenario describing certain types of defects.

The concept of role is used here to encompass all methods that are provided to guide the inspectors during individual reading.

Checklists. Checklists are one of the most concrete indicators in the model. Checklists are instruments for inspectors, helping them to read through the document more analytically and concentrate on the important issues. To provide significant additional value for the review, checklists have to be up-to-date, actively maintained and available to the inspectors all the time. For maximum benefit, checklists ought to be tailored by role, as the role description indicator suggests, or on some other meaningful basis, to keep each checklist manageable in size and understandable.

Examples of checklists may include:

- Universal checklists, taken from (Wiegers 2002), for example.

- Company-specific checklists that have been created from a specific viewpoint (e.g., end-user viewpoint).

Checklists have to be derived from rules and roles. Thus, these indicators are interconnected. Sometimes rules can even be directly used as checklists.

Process data measured. Measurement is the key and prerequisite to all improvement. Without relevant data, past performance cannot be investigated and compared with the current development environment. Data is needed to establish and direct future improvement of the inspection process itself, and development within the organization in general. Measuring helps in benchmarking and comparing projects, tracking process performance to learn whether specific objectives are met, and preventing the same mistakes being made in the future.

The data gathered has to be meaningful, reliable and consistent. This requires a clear definition of what data to collect, when it will be collected, and who will collect it, as well as who will use it and how it will be used. Furthermore, the participants - inspection leaders in particular - have to be trained to collect and deal with the data.

Examples include:

- Number of defects and their classification calculated by an inspection tool.

- Time used for individual checking as reported by the participants.

Data gathering tools can help in automating parts of the measuring process. However, there are metrics that cannot be automatically collected reliably, such as total time used to inspect the document. This is one of the most fundamental indicators, as analysing and improving the process is not possible without reliable data.

Reports. Reports are the essential purpose of data collection. The reports indicator includes all the documentation that is produced as a result of the refinement of the 
measured data. As with measurement, there are two objectives for reporting on inspections: assessing artefact quality and revealing process performance. Furthermore, reports typically include information that can be used in predicting the density and types of defects in similar artefacts in future development.

The information should be as illustrative as possible, as the communication of successful inspections to all personnel is crucial in order to motivate inspectors. Furthermore, senior management needs to be informed of the overall inspection results.

Examples of the reports indicator include:

- Reports on inspection efficiency (defects per hour) or inspector preparation rates (pages per hour).

- An estimate of defects in similar documents to those that have been inspected.

Reports can be tool-based or on paper, depending on the needs of the organization. Reports are separated from the measured process data indicator to emphasize that the data itself is not useful without meaningful interpretation.

Process change requests. As with other software development processes and subprocesses, inspections should be updated when necessary. For example, if meetings are considered too laborious to arrange in a distributed environment, one should examine possible ways to replace meetings with collaboration tools. Gilb \& Graham (1993) state that sticking to a fixed inspection process model and never changing it is a typical reason for the failure of an inspection. The results of the inspections should also be reflected in other development processes.

Examples of the process change requests indicator include:

- An inspection report showing that most defects originate from a certain development phase.

- A decision to use a spreadsheet application instead of unstructured e-mail messages to report issues.

This indicator seldom appears in an unambiguous written form. Instead, it can be found in process improvement suggestions, initiatives taken in the SQA group, the management's reactions to inspection results, or informal discussions between developers.

\subsubsection{Summary}

In addition to the best-known inspection process models, Table 28 includes experience reports on the setting up and operation of different variations of inspection processes in the industry. The reference list is not intended to be comprehensive. Instead, it illustrates that attention has been paid to the indicators identified above in experience reports involving inspections.

These indicators can be identified (with varying terminology) in all comprehensive inspection textbooks. Gilb and Graham (1993), Wiegers (2002) Ebenau and Strauss (1993) and Radice (2002) all address the issues that are described as indicators in the i3 model. 


\section{4}

The articles in Table 28 provide additional evidence on the relevance of the indicators. In these articles, different terminology may be used concerning the indicators, but they all report experiences that promote the use of certain indicators. For example, Jalote and Haragopal (1998) report that data demonstrating the benefits of inspections can be used to overcome difficulties in installing the process. Thus, as Table 28 illustrates, their experience suggests measuring the process (measured process data, indicator number 25) and communicating this data to inspectors and managers (reports, indicator number 26). 
Table 28. Indicators identified in inspection literature.

\begin{tabular}{|c|c|c|c|c|c|c|c|c|c|c|c|c|c|c|c|c|c|}
\hline 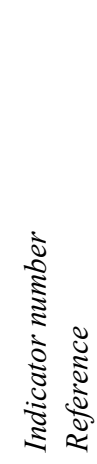 & 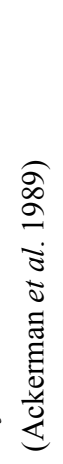 & 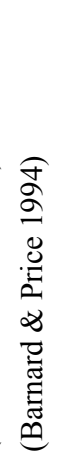 & 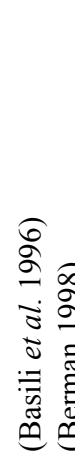 & 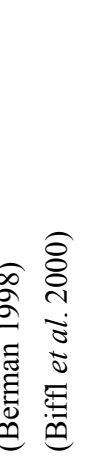 & 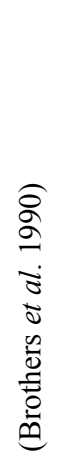 & 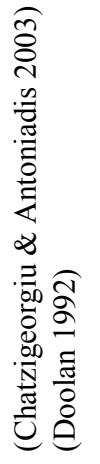 & 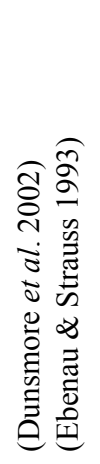 & 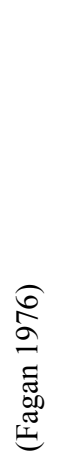 & 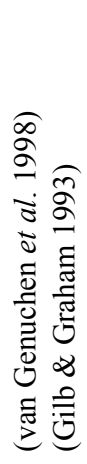 & 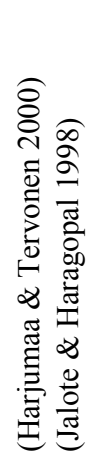 & 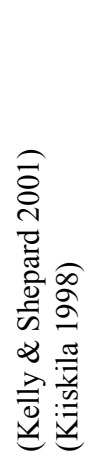 & 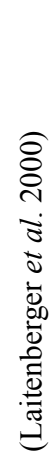 & 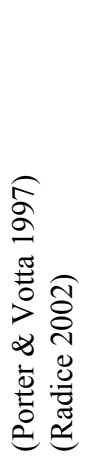 & 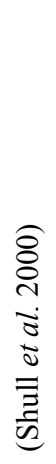 & $\begin{array}{l}\widehat{\approx} \\
\stackrel{\sigma}{\sigma} \\
\frac{\overline{0}}{\overline{0}}\end{array}$ & 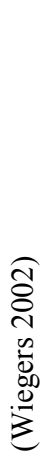 & 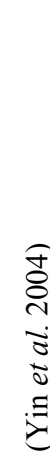 \\
\hline 1 & & & $X$ & $\mathrm{X}$ & & $\mathrm{X}$ & $\mathrm{X}$ & & $X$ & & & & $\mathrm{X}$ & & & $X$ & \\
\hline 2 & $\mathrm{X}$ & & $\mathrm{X}$ & $\mathrm{X}$ & & $\mathrm{X}$ & $\mathrm{X}$ & $X$ & $X$ & & & & $\mathrm{X}$ & & & $\mathrm{X}$ & \\
\hline 3 & & & & & & $\mathrm{X}$ & $\mathrm{X}$ & $X$ & $X$ & & & & $X X$ & & & $\mathrm{X}$ & \\
\hline 4 & $\mathrm{X}$ & & & & & $\mathrm{X}$ & $\mathrm{X}$ & & $X$ & & & & $\mathrm{X}$ & & & $X$ & \\
\hline 5 & & & & & & & X & $X$ & $X$ & & & & $\mathrm{X}$ & & & $X$ & $X$ \\
\hline 6 & $X$ & & & $\mathrm{X}$ & & $\mathrm{X}$ & $\mathrm{XX}$ & $\mathrm{X}$ & $X$ & & $X$ & & $X$ & & & $\mathrm{X}$ & $\mathrm{X}$ \\
\hline 7 & & & & $\mathrm{X}$ & & $\mathrm{X}$ & X & $X$ & $X$ & & & & $\mathrm{XX}$ & & & $X$ & \\
\hline 8 & & & & $\mathrm{X}$ & & $\mathrm{X}$ & $\mathrm{X}$ & & $X$ & & & & X & & & $X$ & \\
\hline 9 & & & & $\mathrm{X}$ & & $\mathrm{X}$ & $\mathrm{X}$ & & $\mathrm{X}$ & & & & $\mathrm{X}$ & & & $\mathrm{X}$ & $\mathrm{X}$ \\
\hline 10 & & & & $\mathrm{X}$ & & & $\mathrm{X}$ & & $\mathrm{X}$ & & & & $\mathrm{X}$ & & & $\mathrm{X}$ & \\
\hline 11 & & & & $\mathrm{X}$ & & $X$ & $\mathrm{X}$ & & $X$ & & & & $\mathrm{X}$ & & & $\mathrm{X}$ & $X$ \\
\hline 12 & & & & $\mathrm{X}$ & & $\mathrm{X}$ & $\mathrm{X}$ & $X$ & X & & & & $\mathrm{X}$ & & & $X$ & $\mathrm{X}$ \\
\hline 13 & & & & & & & X & & $\mathrm{XX}$ & $\mathrm{X}$ & & & $\mathrm{X}$ & & & $\mathrm{X}$ & \\
\hline 14 & $X$ & & & & & & $\mathrm{X}$ & & $\mathrm{X}$ & & $X$ & & $\mathrm{X}$ & $\mathrm{X}$ & & $\mathrm{X}$ & \\
\hline 15 & $\mathrm{X}$ & & $\mathrm{X}$ & & & & $\mathrm{XX}$ & $X$ & $\mathrm{X}$ & & $X$ & $X$ & $\mathrm{X}$ & $\mathrm{X}$ & & $\mathrm{X}$ & \\
\hline 16 & & & & & $\mathrm{X}$ & & & & $\mathrm{X}$ & $X$ & & & $\mathrm{XX}$ & & & $X$ & \\
\hline 17 & & $X$ & & & $\mathrm{X}$ & & & & & $\mathrm{X}$ & & & $\mathrm{XX}$ & & & $X$ & \\
\hline 18 & & & & & & & $\mathrm{XX}$ & $X$ & $\mathrm{X}$ & & & $\mathrm{X}$ & X & & & $X$ & \\
\hline 19 & $\mathrm{X}$ & & $\mathrm{X} X$ & $\mathrm{X}$ & & & $\mathrm{XX}$ & & $\mathrm{X}$ & & & $X$ & X & $\mathrm{X}$ & & $X$ & \\
\hline 20 & & & $\mathrm{XX}$ & $\mathrm{X}$ & & & $\mathrm{XX}$ & $X$ & $\mathrm{X}$ & & & $X$ & $\mathrm{XX}$ & $\mathrm{X}$ & & $\mathrm{X}$ & \\
\hline 21 & $\mathrm{X}$ & & & & & $\mathrm{X}$ & X & & $\mathrm{X}$ & & & $X$ & X & & & $\mathrm{X}$ & \\
\hline 22 & $X$ & & & & & & $X$ & $\mathrm{X}$ & $X$ & & & & $\mathrm{X}$ & & & $X$ & \\
\hline 23 & & & & & & & $\mathrm{X}$ & & $X$ & $\mathrm{X}$ & & & $X$ & $X$ & & $X$ & \\
\hline 24 & $X$ & & $\mathrm{X}$ & $\mathrm{X}$ & & $\mathrm{X}$ & $\mathrm{X}$ & & X & & & & $X$ & $\mathrm{X}$ & $\mathrm{X}$ & $\mathrm{X}$ & \\
\hline 25 & & $X$ & & & & X & $\mathrm{X}$ & $X$ & $\mathrm{XX}$ & $\mathrm{X}$ & & $X$ & $\mathrm{X}$ & & $\mathrm{X}$ & $\mathrm{X}$ & \\
\hline 26 & $X$ & & & & & & $\mathrm{X}$ & $\mathrm{X}$ & $\mathrm{XX}$ & $X$ & & $X$ & $X$ & & $\mathrm{X}$ & $\mathrm{X}$ & \\
\hline 27 & $X$ & & & & & $\mathrm{X}$ & & & X & & $\mathrm{X}$ & & $X$ & $X$ & $\mathrm{X}$ & $\mathrm{X}$ & \\
\hline 28 & $\mathrm{X}$ & & & & & $\mathrm{X}$ & & & $\mathrm{X}$ & & $\mathrm{X}$ & & $X$ & $X$ & $\mathrm{X}$ & $X$ & \\
\hline 29 & $\mathrm{X}$ & $\mathrm{X}$ & $\mathrm{X}$ & $X$ & & $\mathrm{X}$ & $X$ & $\mathrm{X}$ & $\mathrm{X}$ & $X$ & & & $\mathrm{X}$ & $\mathrm{X}$ & $\mathrm{X}$ & $\mathrm{X}$ & \\
\hline
\end{tabular}




\subsection{Assessment and Results}

The ISO/IEC/JTC1/SC7 committee (1992) defines process assessment as follows:

"The disciplined examination of the process used by an organization against a set of criteria to determine the capability of those processes to perform within quality, cost and schedule goals. The aim is to characterize current practice, identifying strengths and weaknesses and the ability of the process to control or avoid significant causes of poor quality, cost and schedule performance."

A disciplined set of criteria for inspection process assessment is defined by the i3 reference model, as was depicted in Figure 24. Activities describe the essence of the process and indicators provide concrete items that are compared to the evidence found in the organization. Figure 24 also illustrates the inputs and outputs for inspection process assessment. Before the actual assessment, its purpose and scope have to be defined. The assessment can be done to initiate an improvement programme within the organization, or just for one project, to estimate its work practices, for example. A quality handbook, process definitions, interviews and actual development work products can be used to gather data on the inspection procedures in use. The assessment produces a profile that can be used to direct improvement and to define precise targets for improvement.

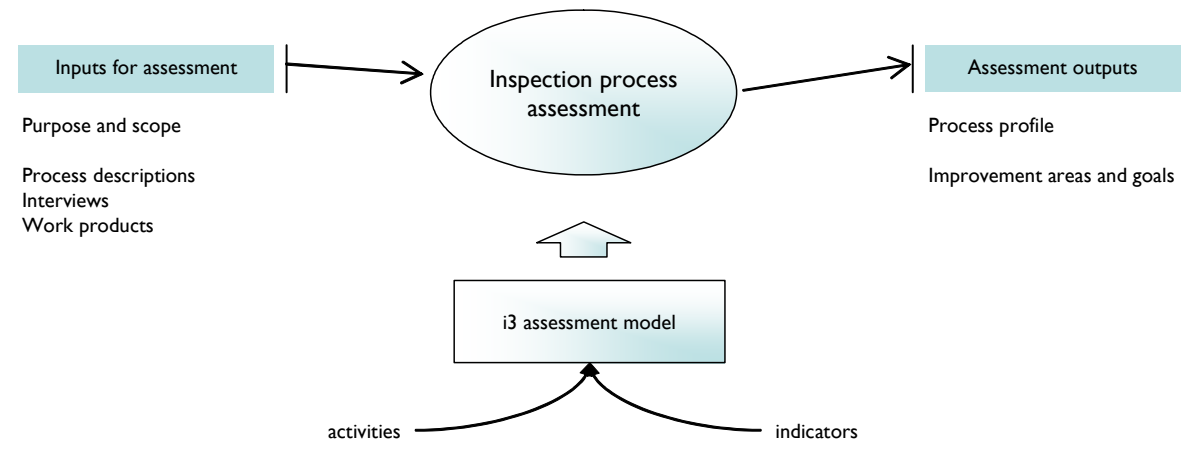

Fig. 26. Assessing the inspection process with the i3 model.

The actual assessment of the capability of the inspection process is carried out during one interview session. To gain objective and relevant insight on the current process status, people from different projects and divisions should participate. According to experiments in five companies (described in paper III), practices and attitudes may be quite different between different teams. This may be caused by numerous reasons and does not directly imply that development is being done carelessly in some teams. People can work on several projects, a team can cooperate with customers that do not practice inspections, or the project can consist of only one or two developers, for example. All these factors make arranging inspections challenging, especially if the company does not have an established quality assurance culture. 
Participants in the assessment interview have to agree on the extent to which the activities defined by the $i 3$ model are realized in the company. Because it is difficult to state objectively how well a certain activity is performed, the evaluation is based on the concrete work products that have been created during the development and that relate to the inspection process. That is, the indicator list of the i3 reference model is used as the basis for the evaluation.

The meaning of each indicator is briefly explained to the participants by the assessor. After that, the relevance of the indicator in the company's context is discussed. If more than one project or division is evaluated in a company that does not have universal quality assurance procedures installed, it is typical that indicators will be interpreted differently between project representatives. A situated definition of the indicator has to be achieved before it can be assessed.

For example, evaluation of the "entry criteria" indicator would be carried out according to the following scenario:

1. The assessor defines "entry criteria" as a clear and unambiguous rule that the inspection leader can use to decide if an inspection can be started. The criteria include checking document quality and inspector availability, for example.

2. Interviewees discuss the meaning of entry criteria and identify elements and tasks in their development project that may relate to the indicator. The assessor clarifies the concept where necessary. The extent to which the indicator should be implemented must also be determined. For example, the entry criteria may be implicit in very small companies where one person manages all inspections, and explicitly written in an inspection handbook in a company that has several projects running simultaneously.

3. Interviewees summarize their views of the entry criteria indicator. They state how the indicator shows in the development process - and how it should show in their situation. If consensus is not achieved, the assessor can ask specifying questions and make his or her own judgment about the existence of the indicator.

All 29 indicators are evaluated. According to the evaluation experiments carried out with the $i 3$ model, this takes two to four hours, depending on the number of participants and the projects that they represent. The evaluation can also be performed for one project only.

Each indicator is ranked on a scale of $0-3$, or $n / a$ if the indicator is not relevant for the organization. The most fundamental indicators, such as the issue list, must always exist and cannot be considered irrelevant. The ranking is recorded in the i3 evaluation form tool, which is a spreadsheet document including all the necessary formulas for the calculation of the capability level. Figure 27 illustrates the evaluation form tool. The first column from the left shows the indicator name and the second column states the score that the indicator has been given in the assessment. For example, the entry decision indicator has been given a score of 2 in this figure.

Figure 27 also depicts the matrix that is used to map indicators to certain activities. The letter $\mathrm{E}$ in the matrix means that the indicator is an enabling indicator for an activity. For example, "entry criteria" is an enabling indicator for activity A.7, "check preconditions for inspection". The letter V means that an indicator is a verifying indicator for an activity. An activity can be both enabling and verifying at the same time (e.g., the "forms" indicator for A.2, "organize the inspection"). 
The numbers in the matrix are computational scores. Verifying indicators are emphasized in the model by giving them a factor of two and if an indicator performs both enabling and verifying roles for an activity, it has a factor of three. The factor of enabling indicators is one. The weighted indicator scores are totalled up and the final score for an activity is then calculated as a percentage value of the maximum score.

The results of the evaluation are communicated immediately to the participants. Results are provided as a simple and illustrative bar graph, with all the indicators mapped to their corresponding activities. The assessor summarizes and explains the results graph that shows the status of each distinct activity of the inspection process. The results graph appears in the topmost part of Figure 27. Each bar depicts the percentage value demonstrating the extent to which the activities are in use within the organization. The percentage is also shown as a number beneath each bar. The "sum" and "max" rows underneath the matrix are numerical expressions of the indicator scores for each activity. 


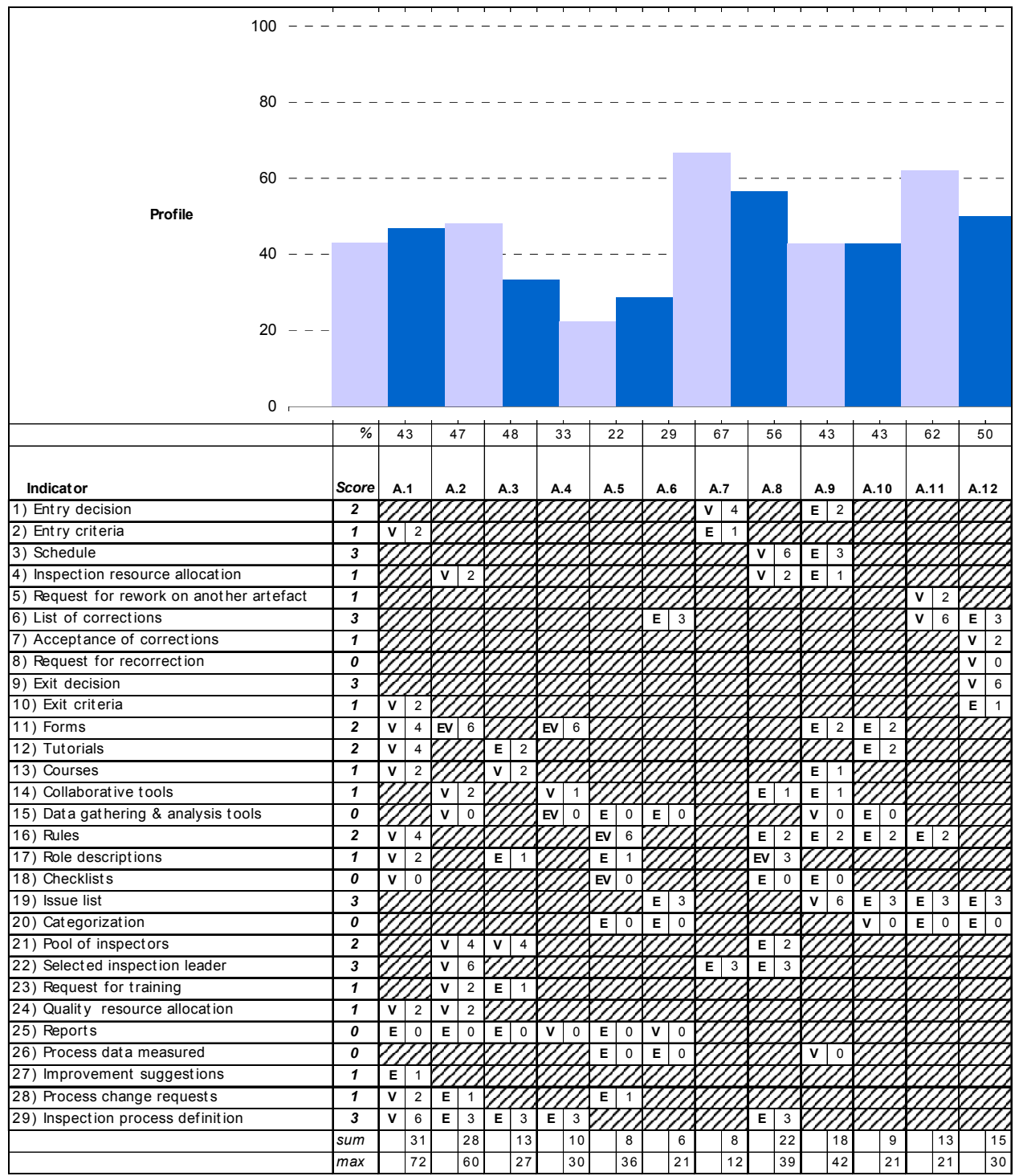

Fig. 27. An example i3 evaluation matrix and results graph.

\subsection{Improvement}

Process improvement methodologies such as CMM (Paulk et al. 1993) and SPICE (Rout 1995) define the fundamental characteristics for high-quality software development. They give guidance for software producers in defining and managing their processes, but do not describe a specific development process, or precise activities for the achievement of 
certain improvements. SPICE includes a process improvement guide, which aims to describe how to use assessment results for SPI.

In the case of the software inspection process, full scale process improvement models provide little help. For example, CMM and SPICE imply that inspections should be implemented to achieve maturity level two, but they do not provide guidelines on what documents to inspect and when to inspect them. Instead, they imply that one should inspect everything.

The i3 model provides a framework for defining an appropriate inspection process model on a case by case basis for different development organizations. Improvement should be tailored and focused as well. Thus, improvement goals are set with respect to the specific organizational needs identified with the i3-based assessment. The improvement is guided by improvement patterns that are described in more detail in Chapter 6.

\subsection{Summary}

The i3 reference model provides a framework for defining inspection processes and evaluating the process capability. The structure of the model is strongly influenced by generic SPI models, in particular the Bootstrap model. This enables compliance to fullscale models and the use of $\mathrm{i} 3$ assessment to trigger extensive improvement activities.

Process evaluation in the $\mathrm{i} 3$ model is not based solely on process descriptions or quality handbooks, because they do not guarantee that the defined process is really followed. The assessment focuses on work products (indicators) and other material created during the development, and evaluation data is gathered through interviews rather than plain documentation.

The assessment procedure and presentation of the results are made as simple as possible to encourage companies with limited resources to employ the method. According to the experiments that have been carried out with the i3 assessment model, company representatives have appreciated the effective organization of the assessment session, and widely agreed with the results that the method has demonstrated. 


\section{The Pattern Approach to Inspection Improvement}

The purpose of a process assessment is to point out weak areas in the software development process. After the assessment, the outcomes and results should be made concrete in a realistic improvement strategy. This is not always a straightforward task. Even the large-scale software process standards, such as SW-CMM or ISO 15504, provide little practical guidance as to exactly which process attributes should be measured, and how to utilize the results. According to the survey carried out by Herbsleb and Goldenson (1996), 67\% of SPI managers would like guidance on how to implement activities defined in SPI models, rather than on what activities to implement.

Concrete guidelines and procedures are necessary for the successful execution of an improvement programme. Especially in smaller companies, quality assurance and improvement resources have to be targeted carefully to the most important parts of the software process (Demirörs et al. 1998, Brodman \& Johnson 1994). With the i3 model, the process patterns approach is taken to guide the improvement.

In the $\mathrm{i} 3$ context, patterns are neither components that are put together to get a process description, nor workflows describing what steps to take to accomplish a certain task. Instead, inspection improvement patterns emphasize the original idea of pattern languages: they attach a set of improvement actions (solutions) to certain improvement targets (problems) in companies that have limited resources (context).

\subsection{Pattern Origins}

The introduction of new practices, methods or tools often originates from somewhere outside the organization itself (Grady \& van Slack 1994). Because this is the first attempt to capture software inspection knowledge in the form of patterns, it is natural that the inspiration and substance for the patterns comes mostly from inspection and SPI literature.

The creation of inspection improvement patterns involves three stages. First, the recurring event that is seen as problematic needs to be identified. For example, because lack of rigor is reported as a problem in several articles, it is clearly a potential basis for a pattern. Next, reported solutions to the problem need to be investigated and attached to 
the problem description. After packaging this knowledge in a reusable form, the pattern can be utilized in the defined situations. These steps are depicted in Figure 28.

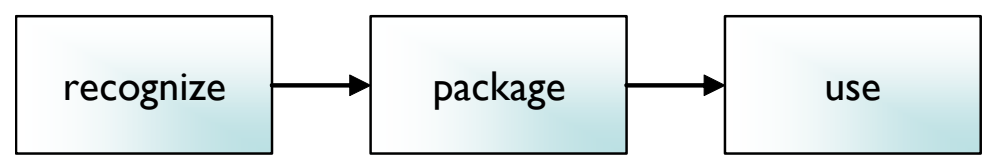

Fig. 28. The pattern creation process.

The inspection improvement patterns introduced here mostly come from cases reported in inspection textbooks and research articles concerning the challenges and typical problems encountered when utilizing inspections. In fact, most inspection textbooks include sections dealing with possible problems. Wiegers (2002) presents a troubleshooting guide for reviews and identifies some special challenges relating, for example, to distributed engineering. Gilb and Graham (1993) outline typical problems and suggest solutions to them. They emphasize the installation of the process and creating a sound basis for it. Radice (2002) lists the deadly sins of inspections and gives some guidance on how to manage problematic situations confronted in the inspection process. Yourdon (1989) provides guidelines for arranging different types of walkthroughs and briefly summarizes possible matters that can go wrong with structured walkthroughs, based on his own experience.

The work is speculative to some degree. Only limited use has been made of these patterns by real-life organizations to enhance their inspection processes. It is difficult to test and validate the patterns by experimentation, because measuring the goodness of certain human-centric activities and processes would be complex and multidimensional and very hard to define. Furthermore, it is difficult to carry out such experiments with tight enough control variables for the effects of a pattern to be isolated from other influencing factors. If a company was ready to make a long-term commitment to such an experiment, it would be possible to get stronger evidence of the effectiveness of the patterns.

The claim that the software inspection improvement pattern language as a whole captures essential knowledge needed to enhance the inspection process has not been thoroughly validated, but has a sound foundation in case studies reported in literature and empirical observations from several experiments carried out in the industry during the course of this study.

The approach taken here is slightly different from, for example, that of Ambler's work (Ambler 1998), in which he classifies process patterns as task, stage and phase process patterns, which describe a series of actions for the development of software. The improvement patterns listed here are not process components, but descriptions of proven (or frequently suggested) solutions to common problems that have emerged when running the process. 


\subsection{Inspection Improvement Pattern Structure}

The structure for inspection improvement patterns is a synthesis of various pattern languages. The Alexandrian form suggests a three-part structure (problem, solution and context), (Gnatz et al. 2002) proposes a larger structure for process patterns (as was illustrated in Chapter 4.5), and (Gamma et al. 1994) uses thirteen elements to document design patterns.

To include all the necessary information but nothing extra, the following sections and characteristics have been defined for inspection improvement patterns:

The Name of the pattern.

The Intent of the pattern, which describes how the inspection process is enhanced if the pattern is used.

The Problem section, which explains the problem that can be addressed by the pattern. The problem is also exemplified. Possible reasons for the problem's occurrence are also discussed briefly.

The Symptoms section, which presents a variety of problems and difficulties. If these have been encountered during inspections, the pattern may be suitable for the given situation. Possible reasons for the problems or inefficiencies are listed. These first parts of the pattern describe the problem, as in design patterns.

The Context section, which describes situations where the pattern fits in.

The Solution section, which represents the body of the suggested solution and a strategy for solving the problems. The procedures are derived from solutions provided by the inspection literature and the i3 inspection capability model.

The Consequences section, which lists possible effects of the improvement actions on other processes and practices.

This structure has been determined after applying the pattern approach in a software company that had just launched inspections and was making an effort to establish the process within the organization. For example, the consequences section was added after the experiment. It was considered necessary to list the consequences in order to understand the implications of applying a pattern, because certain improvements can have organization-wide effects. Furthermore, the action list section was renamed "solution" and dramatically shortened after the experiment, and guidelines in the list were completely rewritten to be more specific. Other parts of the pattern structure have been derived mostly from pattern literature. The formulation here has also taken advantage of ideas from design patterns and process patterns.

\subsection{Usage of Patterns}

The lack of practicable guidelines for process improvement has been recognized. For example, (Niazi et al. 2005) introduces a framework for guiding the implementation of a 
SPI programme. The approach consists of three components: SPI implementation plan, SPI implementation roadmap and SPI implementation model. The framework aims at providing practical advice for the establishment of a process improvement strategy (Niazi et al. 2005).

Appleton (1997) has presented patterns for conducting process improvement. Unlike the patterns presented in this study, Appleton's patterns concern the improvement of the whole development process. Patterns are classified as organizational or process/communication patterns. They attempt to address the most typical problems faced when initiating improvement. For example, the establishment of action teams for each process area that has been selected for improvement can be depicted as a pattern (Appleton 1997).

Utilizing inspection improvement patterns requires the inspection process to be evaluated and the results of the evaluation to be analysed. Figure 29 illustrates the course of actions when using the patterns. After the evaluation, the main goals for the improvement need to be defined. The most suitable patterns for the situation can then be selected from the pattern catalogue. Currently, there are seven patterns in the catalogue, which can ve found in Appendix I.

When choosing an appropriate pattern or patterns, the symptoms sections can be searched to identify problems that match the situation. Finally, prioritization of improvement actions is beneficial, as it is not necessary to carry out all the procedures in the action list - for example, particular items may already have been taken care of. 


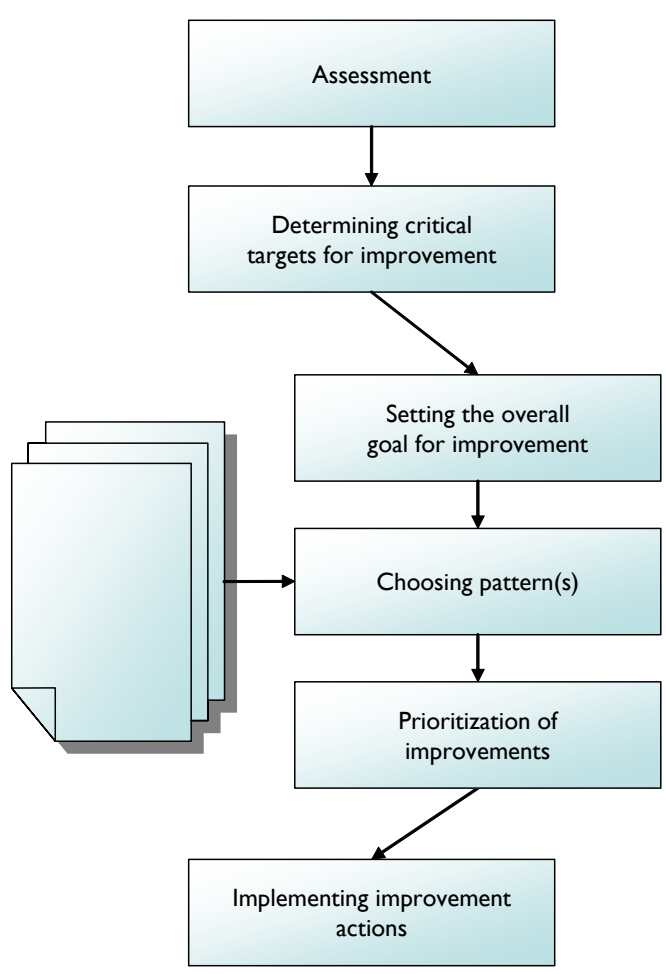

Fig. 29. Using patterns in inspection process improvement

One notable characteristic of a process pattern - or any type of pattern - is that it describes what actions one should perform and how one should perform them, without binding the solution to specific tools or environments. An example concerning the i3 inspection process model should clarify this. The i3 model states that an issue list has to be produced during the process. The important thing is that the list exists, but it does not matter how the list is created or what form it takes. It can be on paper or in a spreadsheet tool. If a pattern was created based on that activity, the pattern would emphasize the existence of the list, not its appearance. This idea is related to the original design patterns, which are independent of implementation languages and tools.

\subsection{Patterns}

The initial pattern catalogue contains seven patterns, which are listed in Table 29. The list is by no means complete. When utilizing these patterns, one can combine improvement actions from several patterns or tailor one pattern to fulfil specific needs. However, if the inspection culture in the organization has not been established yet, these patterns provide default implementations for the improvements. 
Table 29. Inspection improvement patterns.

\begin{tabular}{ll}
\hline Pattern name & The main goal of the pattern \\
\hline Greed & Aims at finding more defects during inspections. \\
Early bird & Aims at finding defects at earlier stages of development. \\
Substance & Aims at finding more serious defects in inspections. \\
Comfort & Aims at making the inspection process easier to run. \\
Promotion & Aims at promoting the process so that it is carried out more often and in larger numbers \\
Wisdom & of projects. \\
Precision & Aims at achieving a more understandable, transparent and effective inspection process.
\end{tabular}

In the following, each pattern is briefly described. Some of the typical problems that have been identified in the inspection literature are grouped into the patterns. Basic solutions to these problems are also outlined. More references that recognize problems and solutions related to certain patterns are listed in the pattern reference summary (Table 30).

\subsubsection{Greed}

Software inspections involve a number of procedures and details that have to be obeyed to ensure the effectiveness of the process. Keeping up to optimal checking rates, efficient meeting practices, and avoiding personal criticism are all essential inspection techniques. Rules and checklists have to be in place and available. Overall planning of the inspection iteration is important (Gilb \& Graham 1993, Wiegers 2002).

Problems will arise if the participants are not adequately prepared. It should be ensured that everybody understands the importance of preparation and follows the process. Before an inspection, the inspection leader should also ensure that all inspectors know how to use checklists and other reading techniques and tools.

Reviews find certain kinds of defects but frequently miss others. Many defects are not caught at all. The process should be followed and defects which escape should be studied in order to identify the parts of development that produce difficult errors. Checklists, roles and perspectives should then be updated to catch those errors (Wiegers 2002).

The role of the inspection leader is also important. To ensure their effectiveness, meetings should be prevented from turning into problem-solving sessions. Material needs to be distributed in time and tools should be available to report and analyse findings. The process should be followed rigorously and there should be enough participants to find all types of errors (Gilb \& Graham 1993).

More defects can be found in inspections when perspectives are used to enable several viewpoints on the document under inspection. Furthermore, setting the viewpoint may help novices to understand the process and the document better (Shull et al. 2000, Shull 1998). To be effective, perspectives need to be tailored to the organization's needs. Even though using perspective-based reading may require more effort, it also enables continuous improvement when reviewer feedback is actively collected. 


\subsubsection{Early bird}

Early defect-detection and avoidance through software reviews can significantly reduce development cost. The best strategy would be to prevent defects getting into the work product in the first place. This is clearly impossible, but inspecting documents that are produced during the early stages of the development cycle may help in achieving higher quality. For example, Siew Hock Ow and Yaacob (1997) suggest that a tool-supported requirements review process reduces the amount of defects at later stages. Similarly, reviewing design documentation helps in avoiding problems in the implementation phase. Thus, finding defects in the early phases of the development cycle is profitable. The further the defects leak when software production goes from phase to phase, the more expensive and fatal the errors will become.

Thus, inspections cannot be arranged at the end of a project, when all the "important" development tasks and phases have been completed. Instead, components should be finished and inspected as early as possible (Chatzigeorgiou \& Antoniadis 2003). If a component has interfaces with other products or is used as a source for another component, its quality should be validated before it enters subsequent phases. Furthermore, the initial quality of a document has to be ensured before inspection to ensure that the inspection itself will be carried out efficiently (Gilb \& Graham 1993).

Inspections do not reveal errors if they are not included in the project plan and reviewers are not provided with resources to carry out their tasks. Reviews or inspections can be included at checkpoints in development cycles. It must be remembered, however, that the inspection itself is a project task requiring resourcing, and not just a milestone (Wiegers 2002).

Emphasizing reviews of early life-cycle products such as requirements and designs may help in following the inspection process and ensuring that inspections are not considered too burdensome later in the project. The effectiveness of the process will suffer dramatically if meetings concentrate on revisiting decisions made in earlier phases (Wiegers 2002).

\subsubsection{Substance}

Experts are typically more effective inspectors than those that do not have expertise in the given domain. An inspector who does not understand the work product cannot contribute much to its quality. This emphasizes the role of planning, as the right people should be selected for inspections. A certification system for inspection leaders may be beneficial in order to ensure that planning is done properly (Gilb \& Graham 1993, Radice 2002)

Discussing minor issues or the style of the work product is a common problem with reviews. This may cause the whole review to become useless, or create irreparable personal clashes between the participants. A set of standards for programming, design and analysis is helpful in order to focus participants on real errors instead of typographic or other minor issues. These standards may be derived from corporate standards, or they may be developed especially for the reviews. It is common that the first reviews 
concentrate on agreeing on these standards, and subsequent reviews run more smoothly (Yourdon 1989).

A common trap is that reviewers focus on style, not on substance. Coding standards and templates for other project documentation may help in avoiding this (Wiegers 2002). Furthermore, the purpose of the inspection needs to be clearly defined before the inspection, and adequate time has to be given for individual preparation. Checklists, the author's overview, and other support should be provided at the beginning of the inspection (Berman 1998).

A number of techniques have been presented for the finding of certain types of defects (e.g., for checking object-oriented code) or to help find the most serious defects. Reading techniques, such as perspective-based reading (Laitenberger et al. 2000), help in concentrating on the most important issues.

The document to be inspected should not contain too many trivial errors that take the inspectors' attention away from serious errors. Thus, the entry criteria have to be checked before inspections. If there are large differences in the quality of the documents, sampling and pre-inspections can be helpful (Thelin et al. 2004).

\subsubsection{Comfort}

It is crucial that participants in the process receive adequate technical and organizational support in carrying out inspection tasks. For example, if reviewers have no access to the source documents needed in inspections, or have poor checklists, efficiency will probably suffer. If inspections have been introduced recently, there can be problems in granting inspectors access rights to all the relevant documents. Programmers do not normally read certain documents, e.g., marketing plans, even though it may be beneficial for the inspection. Defined entry and exit criteria can reduce the need to consult documentation from previous development phases, but usually the inspection leader has to dig out all the necessary documents.

Forms and spreadsheets are needed to report findings and calculate basic metrics, and they constitute the basic set of tools. Several computer tools have been presented to aid inspectors in their job. The experiences reported in (van Genuchten et al. 1997) show that electronic meeting support systems can be used to enhance inspections. Online commenting of the artefact under inspection is demonstrated in (Harjumaa \& Tervonen 2000), for example. Kiiskila (1998) reports that automated metrics collection reduces the work required by members of the review team.

With the proper tools and support for inspectors, the process becomes more effective, distribution is enabled and defect lists and other material can be managed in electronic form. On the other hand, decreasing the effort needed by individual inspectors increases the need for control, thus increasing the effort needed by the inspection leader (Votta 1993).

The problems of manual reviews are discussed, and solutions to their shortcomings proposed by Johnson (1994), for example. Manual reviews are expensive (Russell 1991) and error-prone, as they involve tasks like issue recording and fast-paced logging meetings. Automated tools for such tasks and partial automation of the process may help 
in running the process smoothly. As well as the tools, the process itself can be optimized for the organization (Johnson 1994).

Kelly and Shepard (2004) present a case study showing that a tailored light-weight inspection process can be beneficial when the environment shows reluctance to inspections. Radice (2002) also suggests that small inspection teams need flexibility. (Thelin et al. 2004) suggests that sampling can be used when there is insufficient time to inspect everything. They make use of pre-inspections to identify the areas that need to be focused on during the actual inspections.

\subsubsection{Promotion}

Cultural resistance is one of the typical problems in inspection installation. Until people especially those in management - see concrete figures for costs and benefits, they will probably be sceptical (Gilb \& Graham 1993). If the inspection process gets a good start, resistance will break down quickly. Another issue is lack of management support. If the management is reluctant to allocate adequate resources, time and money for the installation and running of the inspection process, the process will most likely fail. Demonstrations of the method, as well as personal involvement, may help in achieving management commitment. Furthermore, tying the inspection to the overall objectives and business strategy of the company helps in promoting the method (Gilb \& Graham 1993).

The importance of the inspection has to be clarified to the engineers, as well. Allocating time for individuals for review tasks, and providing the necessary data creates an enabling infrastructure for inspections. In addition, emphasizing that people should focus on major issues rather than typographical errors or minor defects helps in making the advantages of the process more visible (Gilb \& Graham 1993).

Preventing personal judgment is vital in order to gain the authors' agreement to reviews. One aspect to this is to keep management separated from the running of the process. Managers should never moderate the inspection. Gaining general agreement and approval for the method from authors is as important as selling the method to the management (Gilb \& Graham 1993). Yourdon (1989) also states the importance of not getting personal.

It is important that people participating in reviews are responsible and know that the management appreciates their participation. People will usually work efficiently and carry out review tasks responsibly if they are motivated and understand what is expected from them (Yourdon 1989).

(Stalhane et al. 2004) report a student experiment showing that poor inspector motivation has negative effects: the document was not properly inspected and few defects were reported. Training is suggested, to solve the motivation problem. Choosing the right people for the inspections and making the objectives and benefits clear to everybody clearly helps in achieving good results.

Berman (1998) identified the need to integrate the inspection process closely with every phase of development, especially with the requirements and design phases. Integrating inspections into project enactment also helps in making inspections a standard development procedure (Chatzigeorgiou \& Antoniadis 2003). Management support for 
the process is crucial and it directly motivates and enables the author to make fixes (Berman 1998).

Fagan (1976) recognizes a problem concerning the difficulty of meeting milestones if there are inadequate human resources to perform inspections. Concentrating on properly performed inspection tasks and reducing the bureaucracy helps make the process effective.

\subsubsection{Wisdom}

Programmers learn from their errors - this shows in inspection efficiency and contributes to the quality of the work product. As people, projects, and products change over time, defect types also change. This means that checklists and inspection practices have to evolve over time and be based on experience data.

There may be problems with checklists when starting inspections, as they tend to be too general before real process data is available (Tervonen 1996). A good idea might be to base the first set of checklists on rules utilized in the development. After the process has been running for a while, checklists can be adjusted according to the knowledge gained.

To find out the optimal checking rates, the costs of running the process and the profitability of inspections, there has to be a method for collecting and storing statistical data about performed inspections. If there is a quality assurance database in use, inspection data may be integrated into that. If this integration seems too difficult, a specific inspection database can be launched (Gilb \& Graham 1993).

The performance of the review suffers if trivial matters are discussed in the meetings. It should be clear to participants which defects are major, which are minor and which are merely questions of style. Similarly, it should be clear how the process is carried out, so that long argumentations about irrelevant issues can be avoided (Yourdon 1989). Defect identification and classification tasks have to be clear to inspectors to ensure that the inspection outcome can be made use of. Data from previous inspections may be valuable. Training, links to documents from previous development phases and reviewing all types of documents all help inspectors (Stalhane et al. 2004).

The inexperience of participants is often the main reason for failed reviews. The people involved in the process have to have knowledge and experience of the procedures and mechanics of the review process itself, and of giving and taking criticism. A written set of procedures for conducting review tasks is needed in order to carry them out effectively (Yourdon 1989).

Reviews that are based on metrics are effective and have a positive impact on product quality. Metrics are also important when trying to reveal errors at early stages of the development (Ogasawara et al. 1996). Measuring the process and collecting feedback from it helps in refining the review and development processes so they become more effective. (Ackerman et al. 1989). 


\subsubsection{Precision}

Running inspections effectively is challenging if there is no clear leadership for the process as a whole. There are usually leaders who will see the importance and profits of new methods and tools, and appointing them as process change agents can help in the installation of the inspection process (Gilb \& Graham 1993).

Inspections should be spread uniformly throughout the development. All documents that will be used as inputs in subsequent development phases should be inspected. Furthermore, inspection tasks need to be carried out fully. Omitting phases of the inspection process can make the whole review ineffective (Gilb \& Graham 1993; Chatzigeorgiou \& Antoniadis 2003).

It is a common error not to follow the defined review process. If this is the case, it is important to find out why. The process may be too complex, organizational inspection policy may be lacking, or quality in general may be given too little attention in project management (Wiegers 2002). When introducing inspections in an organization, existing practices should be taken into consideration to avoid extra work (Gilb \& Graham 1993).

There are a number of details that affect the effectiveness of inspections. Discussions that have nothing to do with the document being reviewed should be separated from actual inspections. A proper overview of the material has to be given to participants, and defined preparation rates have to be followed. Logging and other error-prone tasks can be tool-aided to improve the validity of data (Fagan 1976, Yourdon 1989, Gilb \& Graham 1993, Kiiskila 1998). Participants have to be provided with standards for the documents being reviewed, to reduce the amount of time spent on insignificant issues (Yourdon 1989).

The inspection does not end when all participants have reported their findings. Errors need to be fixed, corrections validated and process improvement needs identified. Sometimes the management is only interested in whether an inspection has been carried out, rather than whether it was carried out effectively (Radice 2002). Furthermore, the fixes will perhaps not be done at all, if there is no data or if it is not understandable to the author (Shirey 1992). To make inspections effective, data gathering and analysis is necessary. Finally, the changes should be inspected too.

Reviews are easily skipped in a hurry, or perceived as slowing the project down. One solution to this is to incorporate inspection tasks into the project plan (Wiegers 2002). Careful planning, carried out as early as possible, is also emphasized by Chatzigeorgiou and Antoniadis (2003). They also suggest implementing and inspecting small components as early as possible, to avoid inspection-overload near deadlines (Chatzigeorgiou \& Antoniadis 2003).

The article (Kingston et al. 2000) concerns an interesting problem with peer reviews: what if reviewers have different or conflicting goals for the process? To succeed in a review, it is crucial that group members generate and share common goals before the inspection. To achieve common agreement, the overview and planning of the review have to be done carefully.

At the organizational level, the training of moderators is important, as their capabilities greatly affect the outcome of the inspection (Kiiskila 1998). Training can also be 
arranged to help participants to use perspectives, scenarios and roles during the checking (Wiegers 2002).

\subsection{Summary of Pattern Sources}

Table 30 summarizes the literature sources that have been used as sources for the initial pattern catalogue. In addition to inspection textbooks that present numerous case studies, research articles reporting problems experienced and their corresponding solutions have been searched through to identify the patterns. 
Table 30. Pattern sources.

\begin{tabular}{|c|c|c|c|c|c|c|c|}
\hline Reference & Greed & $\begin{array}{r}\text { Early } \\
\text { Bird }\end{array}$ & Substance & Comfort & Promotion & Wisdom & Precision \\
\hline (Ackerman et al. 1989) & & $\mathrm{x}$ & & & & & $\mathrm{x}$ \\
\hline (Anderson et al. 2003) & & & & $\mathrm{x}$ & & & \\
\hline (de Almeida et al. 2003) & $\mathrm{x}$ & & $\mathrm{x}$ & & & & \\
\hline (Basili et al. 1996) & & & $\mathrm{x}$ & & & & \\
\hline (Berman 1998) & $\mathrm{x}$ & & $\mathrm{x}$ & $\mathrm{x}$ & $\mathrm{x}$ & & $\mathrm{x}$ \\
\hline (Biffl \& Halling 2003b) & & & & $\mathrm{x}$ & & & \\
\hline (Briand et al. 2004) & & & & & & $\mathrm{x}$ & \\
\hline (Brykczynski 1999) & $\mathrm{x}$ & & $\mathrm{x}$ & & & $\mathrm{x}$ & \\
\hline (Chatzigeorgiou \& Antoniadis & & & & & & & \\
\hline 2003) & & $\mathrm{X}$ & & & $\mathrm{x}$ & & $\mathrm{X}$ \\
\hline (Chowdhury \& Land 2004) & $\mathrm{x}$ & & $\mathrm{x}$ & & & & \\
\hline (Ciolkowski et al. 2003) & & & & & & & $\mathrm{x}$ \\
\hline (Doolan 1992) & & & & & & & $\mathrm{x}$ \\
\hline (Ebenau \& Strauss 1993) & $\mathrm{x}$ & $\mathrm{x}$ & $\mathrm{x}$ & $\mathrm{x}$ & $\mathrm{x}$ & $\mathrm{x}$ & $\mathrm{x}$ \\
\hline (Fagan 1976) & $\mathrm{x}$ & $\mathrm{x}$ & $\mathrm{x}$ & & & & \\
\hline (van Genuchten et al. 1997) & & & & $\mathrm{x}$ & & & \\
\hline (Gilb \& Graham 1993) & $\mathrm{x}$ & $\mathrm{x}$ & $\mathrm{x}$ & $\mathrm{x}$ & $\mathrm{x}$ & $\mathrm{x}$ & $\mathrm{x}$ \\
\hline (Grady \& van Slack 1994) & & & & & & $\mathrm{x}$ & $\mathrm{x}$ \\
\hline (Johnson 1994) & & & & $\mathrm{x}$ & & & \\
\hline (Kelly \& Shepard 2004) & & & & $\mathrm{x}$ & & & $\mathrm{x}$ \\
\hline (Kiiskila 1998) & & & & $\mathrm{x}$ & & $\mathrm{x}$ & $\mathrm{x}$ \\
\hline (Kingston et al. 2000) & & & & & & & $\mathrm{x}$ \\
\hline (Laitenberger 2001) & $\mathrm{x}$ & & $\mathrm{x}$ & & & & \\
\hline (Ogasawara et al. 1996) & & & & & & $\mathrm{x}$ & \\
\hline (Parnas \& Weiss 1985) & & & $\mathrm{x}$ & & & & \\
\hline (Radice 2002) & $\mathrm{x}$ & $\mathrm{x}$ & $\mathrm{x}$ & $\mathrm{x}$ & $\mathrm{x}$ & $\mathrm{x}$ & $\mathrm{x}$ \\
\hline (Sauer et al. 2000) & $\mathrm{x}$ & & $\mathrm{x}$ & & & & \\
\hline (Shirey 1992) & & & $\mathrm{x}$ & & & $\mathrm{x}$ & $\mathrm{x}$ \\
\hline (Shull et al. 2000) & $\mathrm{x}$ & & $\mathrm{x}$ & & & & \\
\hline (Siew Hock Ow \&Yaacob 1997) & & $\mathrm{x}$ & & & & & \\
\hline (Stalhane et al. 2004) & $\mathrm{x}$ & & & & $\mathrm{x}$ & $\mathrm{x}$ & $\mathrm{x}$ \\
\hline (Thelin et al. 2004) & $\mathrm{x}$ & & $\mathrm{x}$ & $\mathrm{x}$ & & & \\
\hline (Weller 1993) & & & $\mathrm{x}$ & & & $\mathrm{x}$ & \\
\hline (Wiegers 2002 ) & $\mathrm{x}$ & $\mathrm{x}$ & $\mathrm{x}$ & $\mathrm{x}$ & $\mathrm{x}$ & $\mathrm{x}$ & $\mathrm{x}$ \\
\hline (Votta 1993) & & $\mathrm{x}$ & & $\mathrm{x}$ & & & $\mathrm{x}$ \\
\hline
\end{tabular}

Sources for the patterns can be compared with the sources of the inspection activities and indicators of the i3 model. A large portion of the literature is the same, as the problems experienced are often presented together with solutions or ideas for solutions. Thus, grouping these reported problems and possible solutions to them into a pattern format seems feasible and has in part happened naturally in individual problems. Formulating 
the problems into a pattern catalogue provides a comprehensive and coherent approach for addressing known problems.

\subsection{A Complete Pattern Example}

\section{Pattern name}

Substance

\section{Intent}

The intent of the Substance pattern is to upgrade the inspection process in order to reveal more serious defects in inspections.

\section{Problem}

The following picture depicts a situation where an inspection uncovers only a couple of minor defects in the document under inspection. Bigger X-marks in the document depict serious defects. Sometimes inspections do not catch these. Instead, typographical or cosmetic errors are found.

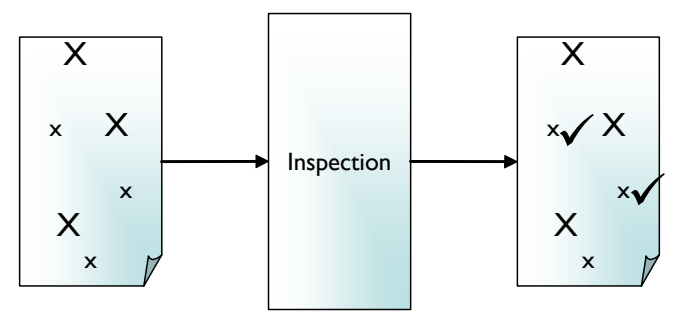

If inspectors do not have knowledge of or experience with the process, it is easiest to concentrate on typographic or other trivial defects and report those. When the purpose and goals of the review are unclear, people perhaps do not have the courage or interest to report any serious defects. To be meaningful, inspections should reveal an adequate number of severe defects.

Poor performance may be caused by insufficient training or inadequate checklist support. Checklists and other reading support techniques should focus attention on the most severe types of errors, and as the process evolves, checklists should be updated. In addition to checklists, it should be made clear how different types of source documents, categorization rules, specialist roles and other inspection guidelines should be used. All these supporting tools help in analysing the document under inspection more carefully and deeply.

Using the Substance pattern should help in revealing more serious issues, as illustrated below. 


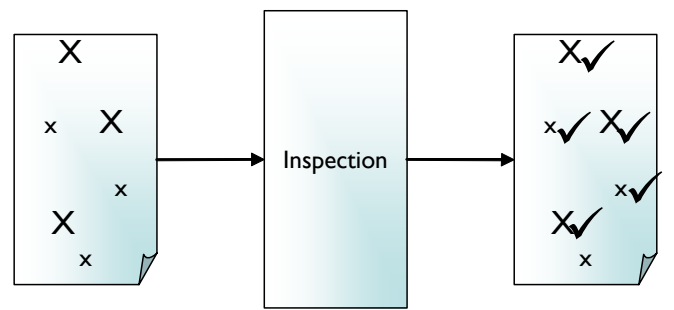

\section{Symptoms}

Symptoms that suggest that use of the Substance pattern would be beneficial include the following:

- Mainly cosmetic defects are reported in inspections.

- No significant modifications are made to inspected artefacts after review.

- The usefulness of inspections is unclear.

\section{Context}

Use the Substance pattern when inspections are arranged regularly, but produce insignificant results. Sometimes the constant absence of major findings may be wrongly interpreted as good quality, and usually inspections are just considered a waste of time, because lists of typographical errors are the most visible outcome.

\section{Solution}

To approach the inspection process with the Substance pattern, upgrade the inspection process as follows:

1. The first thing to check is that the inspection process contains a categorization phase. Attention should be paid to analysis of the causes and types of errors, and inspectors should be aware that this analysis is as significant as revealing the defects. Also, check that in each inspection the participants really do carry out the initial categorization before reporting their findings. Ensure that each inspection leader requires this categorization when planning and arranging inspections.

2. Check that categorization guidelines are up-to-date and reasonable. Check that defect types correspond to the roles and rationales used in the development cycle. For instance, if the software is modularized into user interface, database and telecommunications components, it is natural to classify defects using that division. Other classification principles used must also be relevant. Severity classes that are too finely-grained may be hard to understand, for example. The usual major/minor classification is adequate in most situations. If the categorization system is too complex or too simple, the inspectors will probably get frustrated and will not use it correctly.

3. Update checklists. Irrelevant lists and list items should be dropped. Try to exploit experience data about the usage of checklists. Compare checklists to software engineering rules and guidelines used in the company. Consider defining roles, perspectives and scenarios based on different development aspects: designer, tester, database, communications, etc. 
4. Take advantage of typo lists and other forms that can be used to report non-major findings. This should help inspectors concentrate on more important issues, and emphasizes the reporting of at least some major defects.

5. Train the people involved in inspections. Write a tutorial and make it available. Make sure that inspection leaders have special training. Emphasize the importance of reviews to all inspectors, leaders, and project managers.

6. Check that the material to be inspected is cursorily checked by the inspection leader to ensure the initial quality of the work product.

\section{Consequences}

Utilizing the Substance pattern is not expected to increase the number of defects found. In fact, the total number of reported defects may decrease, as preconditions for inspections are checked more rigorously and inspectors are encouraged to search for more serious faults. Thus, the aim is to reveal more severe issues. Introducing checklists, typo lists and other inspector aids may also initiate tool upgrade and development. It may be beneficial to look at the Comfort pattern, if a considerable amount of effort is needed for such upgrading.

Training must also be arranged to ensure that people understand the meaning and usage of checklists and categorization tools. This training requires a few hours of work and should not require inspectors to sacrifice their free time.

\subsection{Summary}

Patterns encapsulate knowledge concerning inspection process improvement in a reusable and easy-to-follow format. The seven initial patterns constitute the software inspection process improvement pattern catalogue. All the patterns with full descriptions can be found in Appendix I. The catalogue has been tested in the industry with promising results. Pattern structure and contents have been modified according to these experiences, and the current approach seems to provide feasible and concrete instructions for guiding the improvement project. 


\section{Conclusions}

Software quality depends largely on the quality of the process that is used to produce the software. As software nowadays affects all areas of people's lives, the importance of quality is continuously increasing. All software companies, including small ones, have to pay attention to quality issues and ensure the quality of the products they produce.

Small and medium sized companies also have to target their quality improvement efforts carefully, to sustain their competitiveness. They cannot invest large amounts of money on full-scale process improvement programmes that typically require a lot of time and resources.

The main contribution of this study relates to new prospects of the software inspection process, and for its improvement. First, a new view of the inspection process, based on the fundamental activities that are performed during an inspection instead of a series of stages, has been presented. The activity-based process description enables tailoring of the process and its targeted improvement. Second, the study introduces a practicable method for carrying out the improvement. Patterns are especially suitable in companies where resources are limited and full-scale SPI programmes cannot be initiated. Furthermore, the pattern approach represents a promising method for providing more detailed information on how improvements should be carried out than the generic improvement models do. Third, the inspection process currently does not have a very significant role in generic SPI models; this study helps in outlining the importance of inspections. A similar approach could be applied to other software subprocesses to enable their evaluation and improvement. The approach developed and tested in the course of this research provides a meaningful framework for the introduction of an effective quality assurance method in small and medium sized software companies.

\subsection{Research Results and Contributions}

The research consists of several individual constructs and experiments that are addressed in the attached papers. Each paper demonstrates a different aspect of the software inspection process. All the research efforts have one common starting point: they aim at finding a flexible yet efficient means for arranging software inspections in small companies that do not have the resources for burdensome quality assurance methods. 
The research path is described in Table 31, in which the contribution of each individual paper is summarized. The first two papers focus on defining a lightweight inspection process and tool support for such a process. These papers emphasize the benefits of lightweight and computer-supported inspections, but reveal an important process aspect: without a firmly defined process, the introduction of the inspection method and supporting tools is a challenging task. The author is the principal author and contributor in these papers.

The third paper introduces the i3 capability model and reports cases in which the feasibility of the model has been evaluated. In these first experiments, the structure of the capability model is investigated and slightly modified according to the research experiences. The author is co-author of this paper and has contributed especially to the structure of the i3 model.

The next step is reported in the fourth paper, which introduces patterns for guiding improvement after capability evaluation using the i3 model. One case company was looked into, and the main focus was on validating the practicability of the initial pattern catalogue. The fifth paper further explores the pattern approach and introduces refinements to the pattern representations. The author is the principal author and contributor of papers four and five.

The sixth paper is a wrap-up of the research seeking flexible software inspection methods. It summarizes the obstacles that small and medium sized companies come across concerning inspections. It also outlines attitudes towards the inspection method and its degree of utilization within small companies. The results of this investigation are valuable sources for the inspection improvement patterns. The author is co-author in this paper.

Further validation for the i3 model and patterns is presented in the seventh paper, which explores literature on inspections and peer reviews to strengthen the pattern approach. Indeed, the problems addressed with patterns have been confronted in many organizations utilizing inspections. Furthermore, the final format of the inspection improvement patterns is presented in this paper. The author is the sole author of this paper. 
Table 31. Contributions of the papers.

\begin{tabular}{|c|c|c|c|}
\hline Paper & Forum & Theme & Contribution \\
\hline $\begin{array}{l}\text { I } \\
\text { II }\end{array}$ & $\begin{array}{l}\text { HICSS-98, } \\
\text { AI-02 }\end{array}$ & $\begin{array}{l}\text { Computer-supported } \\
\text { software inspection and } \\
\text { flexible forms of inspection }\end{array}$ & $\begin{array}{l}\text { The underlying inspection process needs to be defined } \\
\text { before tools can be used effectively. The process can } \\
\text { be lightweight or exhaustive. }\end{array}$ \\
\hline III & APAQS-01 & $\begin{array}{l}\text { A process model for } \\
\text { software inspection }\end{array}$ & $\begin{array}{l}\text { The model introduced can be used to evaluate } \\
\text { inspection capability without heavy SPI reference } \\
\text { models. Simple and concrete guidance for defining } \\
\text { improvement actions is required. }\end{array}$ \\
\hline IV & PROFES-04 & $\begin{array}{l}\text { Improvement of the } \\
\text { inspection process based on } \\
\text { the process model }\end{array}$ & $\begin{array}{l}\text { Process patterns provide a promising approach for } \\
\text { guiding the improvement and can be used as a starting } \\
\text { point for conducting software process improvement. }\end{array}$ \\
\hline V & QSIC-04 & $\begin{array}{l}\text { Testing the inspection } \\
\text { process improvement } \\
\text { model }\end{array}$ & $\begin{array}{l}\text { Utilizing patterns does have a positive effect on the } \\
\text { effectiveness of the inspection process. Patterns have } \\
\text { to be defined carefully. }\end{array}$ \\
\hline VI & PNSQ-04 & $\begin{array}{l}\text { Summarizing aspects of } \\
\text { inspections in SMEs }\end{array}$ & $\begin{array}{l}\text { The limited resources of small companies have to be } \\
\text { taken into account when planning the inspection } \\
\text { improvement. }\end{array}$ \\
\hline VII & SPIP-05 & $\begin{array}{l}\text { Introducing the } \\
\text { improvement pattern } \\
\text { approach }\end{array}$ & $\begin{array}{l}\text { The literature shows many examples of recurring } \\
\text { inspection problems, which can be combined into a } \\
\text { pattern catalogue. }\end{array}$ \\
\hline
\end{tabular}

This study has outlined the possibilities that software organizations with limited resources have for improvement of their processes. The study is based on the assumption that focusing on specific development subprocesses yields the best cost-benefit ratio if the whole process cannot be addressed. This approach is justified by research results reported in the SPI area. As well as their labour-intensiveness, generic process improvement models have another widely agreed disadvantage: they do not provide practical guidance on how to carry out the improvement. Instead, they concentrate on describing the process elements required to fulfil a certain level of process capability. Especially with limited resources, concrete guidelines would be more practicable. On the other hand, full-scale improvement models attempt to describe all aspects of a development process and including instructions for all subprocesses and all situations would be impossible.

Because software inspections have been proven to produce dramatic quality improvements, it is possible to start the improvement by installing the inspection process, enhancing it later with other quality assurance methods. However, the literature and previous research in the area of software inspections shows that some problems reoccur when organizations are establishing or upgrading inspections or reviews. Experiments with flexible formats for the method seem to confirm that small companies that have little previous experience of systematic quality assurance functions may encounter problems in introducing the inspection method. Inspection improvement patterns have been introduced to capture and incorporate the knowledge from previous research with the goal of eliminating the need to reinvent solutions to these problems.

Introducing inspections in an organization requires a systematic approach. Describing process improvement actions as patterns can make it easier to decide on the concrete 
improvement implementation tasks. The current catalogue of improvement patterns, although not supposed to be complete, provides a good foundation for inspection process improvement. Furthermore, the catalogue offers practical ideas for launching a full-scale process improvement project later.

The entire development process of a company may benefit from the improvement of the inspection process, as some patterns may have organization-wide influences. Upgrading process descriptions, starting a metrics programme, and training developers certainly help in creating a process improvement culture. Highlighting quality issues is always beneficial.

The contributions of this study can be summarized in three main points. First, the study presents a new view of the inspection process, based on the fundamental activities that are performed during an inspection, rather than a series of stages. This enables easier adaptation of the process according to organization-specific needs. The goals for improvement, and targeting this improvement, are also less problematic due to the clearly defined activities. Secondly, the study introduces a practical and manageable method for guiding the improvement actions. The full-scale software process improvement models do not provide detailed information on how improvements should be carried out. The pattern approach represents a promising method for enhancing the reference SPI models with practical implementation guidelines. Thirdly, the inspection process does not hold a process status in generic SPI models, and this study should help in understanding the importance and value of inspections. A similar approach could be applied to other software subprocesses to enable their evaluation and improvement.

\subsection{Limitations and Future Research}

The main focus during this study has been on the inspection process and its straightforward introduction and establishment in small and medium sized organizations. Obviously, installing new processes in an organization causes changes in its overall culture and work practices. In this study, issues of organizational change and the social impacts of the inspection improvement have not been addressed. However, these aspects should not be ignored, and more research is needed in that field.

Investigating the organizational implications of introducing new quality methods would require a longitudinal research in a case organization. Several research reports exist that have analysed the implications of a full-scale SPI programme on product quality. Less information can be found about the effects that SPI has on people's working practices and on organizational infrastructure. The problem of this sort of research is that it is very difficult to measure what proportion of the quality improvement is caused by specific actions, and how much is caused by overall increase in knowledge or personal learning, for example.

More detailed information is also needed on the practical implementation of patterns, to validate the initial patterns further. With limited experimentation, it can be stated that the pattern approach seems to work, but individual patterns need further evaluation. Furthermore, a survey in SME companies that have some experience of inspections would probably reveal additional patterns. 
By definition, a pattern is a pattern only if it reoccurs in real situations. The patterns in the initial catalogue are very fundamental, and the problems described in them are definitely of a recurring nature, according to published inspection research and literature. Finding workable information about the solutions attached to the patterns is more difficult, as solutions are not usually reported in detail. More real-life experimentation would enhance the evidence for the solutions suggested in the patterns. 


\section{References}

Abbott JJ (1997) Software Process Improvement in a Small Commercial Software Company. Proceedings of the 1997 Software Engineering Process Group Conference.

Ackerman AF, Buchwald LS \& Lewsky FH (1989) Software Inspections: An Effective Verification Process. IEEE Software: 6(3): 31-36.

Agerbo E \& Cornils A (1998) How to preserve the benefits of Design Patterns. Proceedings of the $13^{\text {th }}$ conference on Object-oriented programming, systems, languages, and applications: 134143.

Ahern D, Clouse A \& Turner R (2001) CMMI Distilled: A Practical Introduction to Integrated Process Improvement. SEI Series in Software Engineering, Addison Wesley.

Alexander C (1979) The Timeless Way of Building. Oxford University Press.

Alexander C, Ishikawa S \& Silverstein M (1977) A Pattern Language: Towns, Buildings, Construction. Oxford University Press.

de Almeida JR jr, Camargo JB jr, Basseto BA \& Paz SM (2003) Best practices in code inspection for safety-critical software: IEEE Software: 20(3): 56-63.

Ambler SW (1998) Process Patterns: Building Large-Scale Systems Using Object Technology. Cambridge University Press, New York.

Ambler SW (1999) More Process Patterns: Delivering Large-Scale Systems Using Object Technology. Cambridge University Press, New York.

Anderson P \& Teitelbaum T (2001) Software Inspection Using CodeSurfer. Proceedings of the First Workshop on Inspection in Software Engineering.

Anderson P, Reps T, Teitelbaum T \& Zarins M (2003) Tool Support for Fine-Grained Software Inspection. IEEE Software: 20(4): 42-50.

Annala T (2002) Nuorten suomalaisten ohjelmistoyritysten tilannekartoitus, Source Code Finland, http://www.sourcecodefinland.com/liitteet/SCF-yritystutkimus 2002.pdf.

ANSI/IEEE (1989) ANSI/IEEE Std 1028-1988: An American National Standard, IEEE Standards for Software Reviews and Audits.

Appleton B (1997) Patterns for conducting process improvement. PloP'97 conference.

Aurum A, Petersson H \& Wohlin C (2002) State-of-the-art: software inspections after 25 years. Software Testing, Verification and Reliability: 12(3): 133-154.

Barnard J \& Price A (1994) Managing Code Inspection Information. IEEE Software 11(2): 59-69.

Basili V \& Caldiera G (1995) Improving Software Quality by Reusing Knowledge and Experience. Sloan Management Review/Fall. 55-64.

Basili V, Caldiera G \& Rombach HD (1994) Experience Factory. Encyclopaedia of Software Engineering. vol 1: John Wiley \& Sons: 528-532. 
Basili V, Green S, Laitenberger O, Lanubile F, Shull F, Sorumgård S \& Zelkowitz MV (1996) The Empirical Investigation of Perspective-Based Reading. International Journal on Empirical Software Engineering: 2(1): 133-164.

Basili V \& Mills HD (1982) Understanding and Documenting Programs, IEEE Transactions on Software Engineering: 8(3): 270-283.

Beck K (2000) Extreme Programming Explained: Embrace Change. Addison-Wesley.

Beck K \& Cunningham W (1987) Using Pattern Languages for Object-Oriented Programs. OOPSLA Workshop on the Specification and Design for Object-Oriented Programming.

Beck K, Coplien JO, Crocker R, Dominick L, Meszaros G, Paulisch F \& Vlissides J (1996) Industrial Experience with Design Patterns. Proceedings of the $18^{\text {th }}$ International Conference on Software Engineering: 103-114.

Benedicenti L, Succi G \& Vernazza T (1997) From process modeling to domain modeling. ACM SIGAPP Applied Computing Review: 5(2): 28-32.

Berman EF (1998) Software inspections at Fermilab - use and experience. IEEE Transactions on Nuclear Science: 45(4): 1937-1941.

Biffl S \& Halling M (2003a) Investigating the Defect Detection Effectiveness and Cost Benefit of Nominal Inspection Teams. IEEE Transactions on Software Engineering: 29(5): 385-397.

Biffl S \& Halling M (2003b) A Knowledge Management Framework to Support Software Inspection Planning. In: Aurum A, Jeffery R, Wohlin C \& Handzic M (eds.) Managing Software Engineering Knowledge. Springer-Verlag: 231-249.

Biffl S, Halling M \& Kohle M (2000) Investigating the effect of a second software inspection cycle. Cost-benefit data from a large-scale experiment on reinspection of a software requirements document. Proceedings of the First Asia-Pacific Conference on Quality Software: 194-203.

Bisant DB \& Lyle JR (1989) A Two-Person Inspection Method to Improve Programming Productivity. IEEE Transactions on Software Engineering: 15(10): 1294-1304.

Blakely FW \& Boles ME (1991) A Case Study of Code Inspections. Hewlett-Packard Journal: 42(4): 58-63.

Boehm BW (1998) A Spiral Model for Software Development and Enactment. IEEE Computer. 21(5): 61-72.

Bollinger TB \& McGowan C (1991) A Critical Look at Software Capability Evaluations. IEEE Software: 8(4): 25-41.

Borchers JO (2000) A Pattern Approach to Interaction Design. John Wiley \& Sons.

Briand LC, El Emam K, Freimut BG \& Laitenberger O. (2000) A comprehensive evaluation of capture-recapture models for estimating software defect content. IEEE Transactions on Software Engineering: 26(6): 518-40.

Briand LC, Freimut B \& Vollei F (2004) Using multiple adaptive regression splines to support decision making in code inspections. Journal of Systems and Software: 73(2): 205-217.

Brodman JG \& Johnson DL (1994) What Small Businesses and Small Organizations Say About the CMM. International Conference on Software Engineering (ICSE), 331-340.

Brooks FP (1975) The Mythical Man-Month. Addison-Wesley.

Brooks FP (1987) No silver bullet: Essence and accidents of software engineering. IEEE Computer: 20(4): 10-19.

Brothers L, Sembugamoorthy V \& Muller M (1990) ICICLE: Groupware for code inspection, Proceedings of the ACM Conference on Computer Supported Cooperative Work: 169-181.

Brykczynski B (1999) A survey of software inspection checklists. ACM SIGSOFT Software Engineering Notes: 24(1): 82-89.

Buchman CD \& Bramble LK (1995) Three-tiered Software Process Assessment Hierarchy. Software Process - Improvement and Practice 1(2): 99-106. 
Buschmann F, Meunier R, Rohnert H, Sommerland P \& Stal M (1996) Pattern-Oriented Software Architecture - A System of Patterns. John Wiley \& Sons.

Burnstein I (2003) Practical Software Testing. Springer-Verlag.

Butler K (1995) The economics benefits of software process improvement. CrossTalk: 7: 14-17.

Cain B, Coplien J \& Harrison N (1996) Social patterns in productive software organizations. In Annals of Software Engineering: (J. McGregor, Ed.): 2: 259-286.

Chaar JK, Halliday MJ, Bhandari IS \& Chillarege R (1993) In-process evaluation for software inspection and test. IEEE Transactions on Software Engineering: 19(11): 1055-1070.

Chatzigeorgiou A \& Antoniadis G (2003) Efficient Management of Inspections in Software Development Projects. Journal of Information and Software Technology: 45(10): 671-680.

Cheng B \& Jeffery R (1996) Comparing Inspection Strategies for Software Requirements Specifications. Proceedings of the 1996 Australian Software Engineering Conference: 203-211.

Chernak Y (1996) A statistical approach to the inspection checklist formal synthesis and improvement. IEEE Transactions on Software Engineering: 22(12): 866-874.

Chowdhury A \& Land LPW (2004) The impact of training-by-examples on inspection performance using two laboratory experiments. Proceedings of the 2004 Australian Software Engineering Conference: 279-288.

Ciolkovski M, Laitenberger O \& Biffl S (2003) Software Reviews: The State of the Practice. IEEE Software: 20(6): 46-51.

Clough AJ (1992) Software Process Technology. CrossTalk, June/July.

Coad P (1992) Object-oriented patterns. Communications of the ACM: 35(9): 152-159.

Collofello JS \& Woodfield SN (1989) Evaluating the effectiveness of reliability-assurance techniques. Journal of Systems and Software: 9(3): 191-195.

Coplien JO (1995) A Generative Development-Process Pattern Language. In Pattern Languages of Program Design: (J. Coplien \& D. Schmidt, Ed.) Addison-Wesley, New York: 183-237.

Crosby PB. (1979) Quality is Free, The Art of Making Quality Certain. New York: Mentor, New American Library.

Cunningham J \& Finnegan P (2004) Process improvement (PI) programs and information systems: a cross-case analysis of impact. Journal of Information Technology: 19(1), 59-70.

Davenport TH (1993) Process Innovation. Harvard Business School Press.

Davis T (1993) The reuse capability model: a basis for improving an organization's reuse capability. Proceedings of Advances in Software Reusability: 126-133.

Davis A \& Royce W (1994) Rewards of taking the Path Less Traveled. IEEE Software 11(4): 100103.

Deming WE (1982) Out of the Crisis. MIT Press, Cambridge, Mass.

Demirörs E, Demirörs O, Dikenelli O \& Keskin B (1998) Process Improvement Towards ISO 9001 Certification in a Small Software Organization. International Conference on Software Engineering (ICSE), 435-438.

Dewar R, Lloyd AD, Pooley R \& Stevens P (1999) Identifying and communicating expertise in systems reengineering - a Patterns approach, IEEE Proceedings - Software: (146)3: 145-152.

Diaz M \& Sligo J (1997) How Software Process Improvement Helped Motorola. IEEE Software 14(5): 75-81.

Doolan EP (1992) Experiences with Fagan's Inspection Method. Software - Practice and Experience: 22(2): 173-182.

Drummond H (1996) Escalation in Decision-Making: The Tragedy of Taurus. Oxford University Press.

Dunsmore A, Roper M \& Wood M (2002) Further Investigations into the Development and Evaluation of Reading Techniques for Object-Oriented Inspection. Proceedings of the 24th International Conference on Software Engineering: 47-57. 
Dunsmore A, Roper M \& Wood M (2003) Practical Code Inspection Techniques for ObjectOriented Systems:An Experimental Comparison. IEEE Software: 20(4): 21-29.

Dybå T (2000) An Instrument for Measuring the Key Factors of Success in Software Process Improvement. Empirical Software Engineering: 5(4): 357-390.

Dybå T (2003) Factors of Software Process Improvement Success in Small and Large Organizations: An Empirical Study in the Scandinavian Context. Proceedings of the $9^{\text {th }}$ European Software Engineering Conferene: 148-157.

Dybå T \& Moe NB (1999) Rethinking the Concept of Software Process Assessment. Proceedings of the European Software Process Improvement Conference.

Ebenau RG \& Strauss S (1993) Software Inspection Process. McGraw-Hill.

El Emam K (1997) Spice: The Theory and Practice of Software Process Improvement and Capability Determination. IEEE Computer Society Press.

El Emam K, Laitenberger O \& Harbich T (2000) The application of subjective estimates of effectiveness to controlling software inspections. Journal of Systems and Software: 54(2): 119136.

Fagan M (1976) Design and Code Inspections to Reduce Errors in Program Development. IBM Systems Journal 3: 182-210.

Fagan M (1986) Advances in Software Inspection. IEEE Transactions on Software Engineering. 12(7): 744-751.

Fenton EF, Neil M (1999) A Critique of Software Defect Prediction Models. IEEE Transactions on Software Engineering 25(5): 675-689.

Fowler M (1997) Analysis Patterns - Reusable Object Models. Addison-Wesley.

Fredj M \& Roudies O (1999) A pattern based approach for requirements engineering. Proceedings of the Tenth International Workshop on Database and Expert Systems Applications: 310-314.

Galin D (2004) Software Quality Assurance - From theory to implementation. Pearson Education Limited.

Gamma E, Helm R, Johnson R \& Vlissides J (1994) Design Patterns - Elements of Reusable Object-Oriented Software. Addison-Wesley, USA.

van Genuchten M, Cornelissen W \& van Dijk C (1997) Supporting inspections with an electronic meeting system. Journal of Management Information Systems: 14(3): 165 - 178.

German DM \& Cowan DD (2000) Towards a unified catalog of hypermedia design patterns. Proceedings of the 33rd Annual Hawaii International Conference on System Sciences.

George E \& Janiszewski S (2003) Optimizing Software Inspections with Statistical Quality Techniques. Software Quality Professional: 6(1): 16-29.

Geyres S, Bazzana G \& Deler G (1997) Exchanging SPI Experience Across SMEs by Internet Conferencing. Proceedings of the SPI, Barcelona, Spain.

Gilb T \& Graham B (1993) Software Inspection. Addison-Wesley Longman Ltd, Essex, England.

Gintell J, Arnold J, Houde M, Kruszelnicki J, McKenney R \& Memmi G (1993) Scrutiny: A collaborative inspection and review system. Proceedings of the Fourth European Software Engineering Conference.

Gnatz M, Marschall F, Popp G, Rausch A \& Schwerin W (2002) Towards a tool support for a Living Software Development Process. Proceedings of the $35^{\text {th }}$ Hawaii International Conference on System Sciences: 1529-1537.

Gnatz M, Marschall F, Popp G, Rausch A \& Schwerin W (2001) Towards a Living Software Development Process based on Process Patterns. Proceedings of the $8^{\text {th }}$ European Workshop on Software Process Technology: Lecture Notes in Computer Science 2077, Springer: 182-202.

Grady RB \& Van Slack T (1994) Key Lessons in Achieving Widespread Inspection Use. IEEE Software 11(4): 46-57.

Grady RB (1997) Successful Software Process Improvement. Prentice Hall PTR, Upper Saddle River, NJ. 
Guerrero F \& Eterovic Y (2004) Adopting the SW-CMM in a Small IT Organization. IEEE Software: 21(3): 29-35.

Haley TJ (1996) Software Process Improvement at Raytheon. IEEE Software: 13(6): 33-41.

Hammer M (1996) Beyond Reengineering: How the Process-Centered Organization Will Change Our Work and Our Lives. HarperCollins, New York.

Harjumaa L (2003) Distributed Software Inspections - An Experiment with Adobe Acrobat. Proceedings of the IASTED International Conferenceon Computer Science and Technology: 2631.

Harjumaa L \& Tervonen I (2000) Virtual software inspections over the internet. Proceedings of the third ICSE workshop on software engineering over the internet: 30-40.

Harrison NB (1996) Organizational Patterns for Teams. Pattern Languages of Program Design 2. Addison-Wesley.

Hedberg H (2004) Introducing the Next Generation of Software Inspection Tools. Proceedings of the 5th International Conference of Product Focused Software Process Improvement: 234-247.

Heires JT (2001) What I did last summer: a software development benchmarking case study. IEEE Software: 18(5): 33-39.

Herbsleb J, Carleton A, Rozum J, Siegel J \& Zubrow D (1994) Benefits of CMM-based Software Process Improvement: Executive Summary of Initial Results. Special report. CMU/SEI-94-SR013.

Herbsleb JD \& Goldenson DR (1996) A systematic survey of CMM experience and results. Proceedings of the $18^{\text {th }}$ International Conference on Software Engineering: 323-330.

Herrmann T, Hoffmann M, Jahnke I, Kienle A, Kunau G, Loser K-U \& Menold N (2003) Concepts for Usable Patterns of Groupware Applications. Proceedings of the 2003 international ACM SIGGROUP conference on Supporting group work: 349-358.

Hevner AR, March ST, Park J \& Ram S (2004) Design science in information systems research. MIS Quarterly: 28(1): 75-105.

Horvat RV, Rozman I \& Györkös J (2000) Managing the Complexity of SPI in Small Companies. Software Process - Improvement and Practice 5(1): 45-54.

Huang H \& Zhang S (2003) Hierarchical Process Patterns: Construct Software Processes in a Stepwise Way. International Conference on Systems, Man and Cybernetics: 1353-1358.

Humphrey WS (1989) Managing the Software Process. Addison-Wesley, Reading, Mass.

Humphrey WS \& Sweet WL (1987) A Method for Assessing the Software Engineering Capability of Contractors. Preliminary Version. CMU/SEI-87-TR-23. Software Engineering Institute. Carnegie-Mellon University.

Höst M \& Johansson C (2000) Evaluation of Code Review Methods through Interviews and Experimentation. Journal of Systems and Software: 52(2-3): 113-120.

Ishikawa K (1985) What Is Total Quality Control? The Japanese Way. Prentice-Hall.

ISO/IEC/JTC1/SC7 (1992) The Need and Requirements for a Software Process Assessment Standard, Study Report, issue 2.0.

Jacobs D (2004) Accelerating Process Improvement Using Agile Techniques. Crosstalk: March: 49.

Jalote P \& Haragopal M (1998) Overcoming the NAH syndrome for inspection deployment. Proceedings of the 20th International Conference on Software Engineering: 371-378.

Johnson DL \& Brodman JG (1996) Realities and Rewards of Software Process Improvement. IEEE Software: 13(6): 99-101.

Johnson DL \& Brodman JG (1998) Applying the CMM to Small Organizations and Small Projects. Proceedings of the Software Engineering Process Group Conference (SEPG).

Johnson PM (1994) An instrumented approach to improving software quality through formal technical review. Proceedings of the 16th International Conference on Software Engineering: 113-122. 
Johnson PM \& Tjahjono D (1998) Does every inspection really need a meeting? Empirical Software Engineering: 3(1): 9-35.

Johnson RE (1992) Documenting Frameworks using Patterns. Proceedings of the Conference on Object Oriented Programming Systems Languages and Applications: 63-76.

Juran J (1951) Quality Control Handbook. McGraw-Hill.

Kautz K, Hansen HW \& Thaysen K (2000) Applying and Adjusting a Software Process Improvement Model in Practice: The Use of IDEAL Model in a Small Software Enterprise. Proceedings of the $22^{\text {nd }}$ International Conference on Software Engineering: 626-633.

Kelly DP \& Culleton (1999) Process Improvement for Small Organizations. IEEE Computer: 32(10): 41-47.

Kelly DP \& Shepard T (2002) Qualitative observations from software code inspection experiments. Proceedings of the 2002 conference of the Centre for Advanced Studies on Collaborative research: 5-16.

Kelly DP \& Shepard T (2001) A case study in the use of defect classification in inspections. Proceedings of the 2001 conference of the Centre for Advanced Studies on Collaborative research.

Kelly DP \& Shepard T (2004) Task-directed software inspection. Journal of Systems and Software: 73(2): 361-368.

Kelly JC, Sherif JS \& Hops J (1992) An Analysis of Defect Densities Found During Software Inspections. Journal of Systems and Software: 17(2): 111-117.

Kiiskila J (1998) Practical aspects on the assessment of a review process. Proceedings of the $24^{\text {th }}$ Euromicro Conference: 867-870.

Kingston G, Jeffery R \& Huang W (2000) An explanatory study on the goal alignment problem in joint software reviews. Proceedings of the 2000 Australian Software Engineering Conference: 63-72.

Kitchenham B, Kitchenham A \& Fellows J (198) The effects of inspections on software quality and productivity. Technical Report 1, ICL Technical Journal.

Knight JC \& Myers EA (1991) Phased Inspections and their Implementation. ACM SIGSOFT Software Engineering Notes: 16(3): 29-35.

Knight JC \& Myers EA (1993) An Improved Inspection Technique. Communications of the ACM: 36(11): 51-61.

Koomen T \& Pol M (1999) Test Process Improvement. Addison-Wesley.

Kruchten P (2000) The Rational Unified Process: An Introduction. $2^{\text {nd }}$ edition. Addison-Wesley.

Kuvaja P, Similä J, Krzanik L, Bicego A, Saukkonen S \& Koch G (1994) Software Process Assessment \& Improvement - The BOOTSTRAP Approach. Blackwell Publishers, Oxford, UK.

Laitenberger O (2001) Cost-effective Detection of Software Defects through Perspective-based Inspections. Empirical Software Engineering: 6(1): 81-84.

Laitenberger O, Atkinson C, Schlich M \& El Emam K (2000) An Experimental Comparison of Reading Techniques for Defect Detection in UML Design Documents. The Journal of Systems and Software: 53(2): 183-204.

Laitenberger O \& DeBaud J (2000) An Encompassing Life-Cycle Centric Survey of Software Review. Journal of Systems and Software: 50(1): 5-31.

Landay JA \& Borriello G (2003) Design Patterns for Ubiquitous Computing. IEEE Computer: 36(8): 93-95.

Lano K \& Malik N (1997) Reengineering Legacy Applications using Design Patterns. Proceedings of the 8th International Workshop on Software Technology and Engineering Practice: 326-338.

Lepasaar M, Varkoi T \& Jaakkola H (2001) Models and Success Factors of Process Change. In Proceedings of Third International Conference of Product Focused Software Process Improvement: 68-77. 
Lukka K (2003) The constructive research approach. In: L. Ojala, O-P. Hilmola. (eds.) Case study research in logistics. Publications of the Turku School of Economics and Business Administration, Series B 1: 2003: 83-101.

Macdonald F (1998) Computer supported software inspection. PhD Thesis, University of Strathclyde, Glasgow.

MacFarland R (2001). Case Study of an Improvement Program Featuring Reviews and Inspections. Software Quality Professional: 3(3): 26-33.

Mahemoff MJ \& Johnston LJ (1998) Principles for a usability-oriented pattern language. Proceedings of the Australasian Computer Human Interaction Conference: 132-139.

Mahon A (1996) Groupware and the web. American Programmer: August: 19-22.

Mann ML \& Rising L (2005) Fearless Change: Patterns for introducing new ideas. AddisonWesley.

March ST \& Smith GF (1995) Design and natural science research on infromation technology. Decision Support Systems: 15(4): 251-266.

Mashayekhi V, Drake JM, Tsai WT \& Reidl J (1993) Distributed, collaborative software inspection. IEEE Software: 5: 66-75.

Mashayekhi V, Feulner C \& Reidl J (1994) CAIS: Collaborative Asynchronous Inspection of Software. Proceedings of the second ACM SIGSOFT Symposium on the Foundations of Software Engineering.

May D \& Taylor P (2003) Knowledge management with patterns. Communications of the ACM: 46(7): 94-99.

McConnell S (1993) Code Complete - A Practical Handbook of Software Construction.

McConnell S (2002) Real Quality for Real Engineers. IEEE Software: 19(2): 5-7.

McFeeley B (1996) IDEAL: A User's guide for software process improvement. CMU/SEI-96-HB001. Software Engineering Institute.

Moore J, Inder R, Chung P, Macintosh A \& Stader J (2000) Combining and Adapting Process Patterns for Flexible Workflow. Proceedings of the $11^{\text {th }}$ Intenational Workshop on Database and Expert Systems Applications: 797-801.

NASA (1999) Mars Climate Orbiter Mishap Investigation Board Phase I Report. Available: ftp://ftp.hq.nasa.gov/pub/pao/reports/1999/MCO_report.pdf.

Natwick G, Draper G \& Bearden L (1999) Software Mini-Assessments: Process and Practice. Crosstalk: october: 10-14.

Niazi M, Wilson D \& Zowghi D (2005) A maturity model for the implementation of software process improvement: an empirical study. Journal of Systems and Software: 74(2): 155-172.

Nunamaker J, Chen M \& Purdin T (1990) Systems development in information systems research. Journal of Management Information Systems: 7(3): 89-106.

O’Neill (1997) Issues in Software Inspection. IEEE Software 14(1): 18-19.

Ogasawara H, Yamada A \& Kojo M (1996) Experiences of software quality management using metrics through the life-cycle. Proceedings of the 18th International Conference on Software Engineering: 179-188.

Otoya S \& Cerpa N (1999) An Experience: A Small Software Company Attempting to Improve its Process. In Proceedings of the Software Technology and Engineering Practice. Pittsburgh, USA: 153-160.

Parnas DL \& Weiss DM (1985) Active Design Reviews: Principles and Practices. Proceedings of the 8th International Conference on Software Engineering: 132-136.

Paulk MC (1998) Using the Software CMM in Small Organizations. International Conference on Software Quality, 350-361.

Paulk MC (1995) How ISO 9001 compares with the CMM. IEEE Software. 12(1): 74-83.

Paulk MC, Curtis B, Chrissis MB, Weber CV (1993) Capability maturity model, version 1.1. IEEE Software 10: 18-27. 
Peirce CS (1931) Collected papers. Harvard University Press.

Petersson H (2001) Individual reviewer contribution to the effectiveness of software inspection teams. Proceedings of Australian Software Engineering Conference: 160-168.

Pitterman B (2000) Telcordia technologies: the journey to high maturity. IEEE Software: 17(4): 8996.

Porter A, Siy H, Mockus A \& Votta L (1998) Understanding the Sources of Variation in Software Inspections. ACM Transactions on Software Engineering and Methodology 7(1): 41-79.

Porter A, Votta L (1997) What Makes Inspections Work? IEEE Software 14(6): 99-102.

Porter A, Votta L \& Basili V (1995) Comparing Detection Methods for Software Requirements Inspections: A Replicated Experiment. IEEE Transactions of Software Engineering 21(6): 563575.

Radice RA (2002) High Quality Low Cost Software Inspections. Paradoxicon Publishing, Andover, USA.

Richardson I (2002) SPI Models: What Characteristics are Required for Small Software Development Companies? Software Quality Journal 10(2): 101-114.

Rico DF (2002) How to estimate ROI for inspections, PSP, TSP, SW-CMM, ISO 9000, and CMMI. The DoD SoftwareTech News 5: 23-31.

Riehle D \& Zullighoven H (1996) Understanding and using patterns in software development. Theory and Practice of Object Systems: 2(1): 3-13.

Rodgers TL \& Dean DL (1999) Process Maturity and Inspector Proficiency: Feedback Mechanisms for Software Inspections. Proceedings of the 32nd Hawaii International Conference on System Sciences.

Rossi G Schwabe D \& Garrido A (1997) Design Reuse in Hypermedia Applications Development. Proceedings of the Eighth ACM Conference on Hypertext, Hypertext Design: 57-66.

Rossi G, Schwabe D \& Lyardet F (1999) Improving web information systems with navigational patterns. Proceedings of the 8th International World Wide Web Conference: W3C, Elsevier.

Rout TP (1995) SPICE: A Framework for Software Process Assessment. Software Process Improvement and Practice, pilot issue: 57-66.

Rout TP (2000) The Rapid Assessment of Software Process Capability. International Conference on Software Process Improvement and Capability dEtermination (SPICE): 47-55.

Royce W (1970) Managing the Development of Large Software Systems: Concepts and Techniques. Proceedings of the IEEE WESCON: 1-9.

Russell GW (1991) Experience with Inspection in Ultralarge-Scale Developments. IEEE Software: 8(1): 25-31.

Sakamoto K, Kishida K, Nakakoji K (1996) Cultural Adaptation of the CMM: A Case Study of a Software Engineering Process Group in a Japanese Manufacturing Company. Fugetta A, Wolf A, Eds. Software Process. John Wiley \& Sons Ltd, West Sussex: 137-154.

Sakamoto K, Nakakoji K, Takagi Y, Niihara N (1998) Toward Computational Support for Software Process Improvement Activities. International Conference on Software Engineering: 22-31.

Salzmann C \& Maraist J, (1997) A Pattern-Based Approach for a Flexible, Self-Extending Document System. Proceedings of the $24^{\text {th }}$ International Conference on Technology of ObjectOriented Languages: 215-221.

Satpahty M \& Harrison R (2002) A Typed Generic Process Model for Product Focused Process Improvement. Proceedings of the 26th International Computer Software and Applications Conference on Prolonging Software Life: Development and Redevelopment: 379-384.

Sauer C, Jeffery R, Land L \& Yetton P (2000) The Effectiveness of Software Development Technical Reviews - A Behaviourally Motivated Program of Research, IEEE Transactions on Software Engineering: 26(1): 1-14.

Schmidt D (1995) Experience using design patterns to develop reuseable object-oriented communication software. Communications of the ACM: 38(19): 65-74. 
Schneider GM, Martin J \& Tsai WT (1992) An experimental study of fault detection in user requirements documents. ACM Transactions on Software Engineering and Methodology: 1(2): 188-204.

Sembugamoorthy V \& Brothers L (1990) ICICLE: Intelligent code inspection in a C language environment. Proceedings of the Fourteenth Annual International on Computer Software and Applications Conference: 146-154.

Seruca I \& Loucopulos P (2003) Towards a Systematic Approach to the Capture of Patterns within a Business domain. Journal of Systems and Software 67(1): 1-18.

Sheard SA, Zampella S \& Truesdale AJ (2004) Ten Key Techniques for Process Improvement Consulting in a Challenging Environment. Crosstalk: february: 11-13.

Shewhart WA (1931) Economic control of quality of manufactured product. Van Nostrand.

Shirey GC (1992) How Inspections Fail. Proceedings of the $9^{\text {th }}$ International Conference on Testing Computer Software: 151-159.

Shull F (1998) Developing Techniques for Using Software Documents: A Series of Empirical Studies, doctoral dissertation, Computer Science Dept., Univ. of Maryland: Available: http://www.cs.umd.edu/projects/SoftEng/ESEG/papers/postscript/shull_diss.ps.gz.

Shull F, Rus I \& Basili V (2000) How perspective-based reading can improve requirements inspections. IEEE Computer: 33(7): 73-79.

Siew Hock Ow \& Yaacob MH (1997) A study on the requirements review process in software development: problems and solutions. Proceedings of The Twenty-First Annual International Computer Software and Applications Conference: 542-547.

Simon J (1999) Software process identification: A case study using ISO/IEC 12207 software life cycle process standard. In Better Software Practice for Business Benefit (eds: Messnarz R \& Tully C): IEEE Computer Society: 303-315.

Sitra (2001) NetCenter selvitys. Available: http://www.sitra.fi/Julkaisut/osku/osku6.pdf.

Software Engineering Institute (1994) Benefits of CMM-Based Software Process Improvement: Initial Results (CMU/SEI-94-TR-13). Software Engineering Institute, Carnegie-Mellon University.

Software Quality Engineering (2005) Home page. http://www.sqe.com/.

van Solingen R (2004) Measuring the ROI of Software Process Improvement. IEEE Software: 21(3): 32-38.

Sommerville I \& Sawyer P (1997) Requirements Engineering - A Good Practice Guide. John Wiley \& Sons.

Soukup J (1995) Implementing Patterns. (Coplien JO \& Schmidt DC eds) Pattern languages of Program Design. Addison-Wesley.

Stalhane T, Kutay C, Al-Kilidar H \& Jeffery R (2004) Teaching the process of code review. Proceedings of the 2004 Australian Software Engineering Conference: 271-278.

Stein M, Riedl J, Harner SJ \& Mashayekhi V (1997) A case study of distributed, asynchronous software inspection. Proceedings of the 19th International Conference on Software Engineering: 107-117.

Stein M, Heimdahl MPE \& Riedl JT (1999) Enhancing Annotation Visibility for Software Inspection. Proceedings of the Automated Software Engineering Conference: 243-246.

Stevens P \& Pooley R (1998) Systems Reengineering Patterns. Proceedings of the 6th ACM SIGSOFT international symposium on Foundations of software engineering: 17-23.

Störrle H (2001) Describing Process Patterns with UML. European Workshop on Software Process Technology, LNCS, Springer-Verlag: 0173-0181.

Taylor P (1998) Capable, Productive and Satisfied - Patterns for Productivity. Proceedings of the PLoP'98 Conference.

Tervonen I (1996) Consistent Support for Software Designers and Inspectors. Software Quality Journal: 5(3): 221-229. 
Thelin T, Petersson H \& Wohlin C (2001) Sample-Driven Inspection. Proceedings of the 1st Workshop on Inspection in Software Engineering: 81-91.

Thelin T, Runeson P \& Wohlin C (2003) An experimental comparison of usage-based and checklist-based reading. IEEE Transactions on Software Engineering: 29(8): 687-704.

Thelin T, Petersson H, Runeson P \& Wohlin C (2004) Applying sampling to improve software inspections. Journal of Systems and Software: (73)2: 257-269.

Tian J (2001) Quality Assurance Alternatives and Techniques: A Defect-Based Survey and Analysis. Software Quality Professional: 3(3): 6-18.

Travassos GH, Shull F, Fredericks M \& Basili VR (1999) Detecting Defects in Object Oriented Designs: Using Reading Techniques to Increase Software Quality. Conference on ObjectOriented Programming, Systems, Languages, and Applications (OOPSLA): 225-237.

Tyran CK \& George JF (2002) Improving Software Inspections with Group Process Support. Communications of the ACM: 45(9): 87-92.

Waldstein NS (1974) The Walk-Thru - A Method of Specification, Design and Review. Technical Report TR 00.2536, IBM Corporation. Poughkeepsie, NY.

Wang YH, Mei H \& Shao WH (1997) Experience Report: Using Design Patterns in the Development of JB System. Proceedings of the $24^{\text {th }}$ International Conference on Technology of Object-Oriented Languages: 199-204.

Weinberg GM (1971) The Psychology of Computer Programming. Van Nostrand Reinhold, NY.

Weller EF (1992) Experiences with Inspections at Bull HN Information System. Proceedings of the $4^{\text {th }}$ Annual Software Quality Workshop.

Weller EF (1993) Lessons from Three Years of Inspection Data. IEEE Software: 10(5): 38-45.

Wiegers KE (2002) Peer Reviews in Software - A Practical Guide. Addison-Wesley.

Wiegers KE (1998) Read My Lips: No New Models! IEEE Software: 15(5): 10-13.

Wong YK (2003) An exploratory study of software review in practice. Proceedings of the Portland International Conference on Management of Engineering and Technology: 301-308.

Votta LG (1993) Does Every Inspection Need a Meeting? ACM Software Engineering Notes: 18: 107-114.

Yamamura G (1999) Process improvement satisfies employees. IEEE Software: 16(5) : 83-85.

Yin Z, Dunsmore A \& Miller J (2004) Self-assessment of performance in software inspection processes. Information and Software Technology: 46(3): 185-194.

Yourdon E (1989) Structured Walkthroughs. $4^{\text {th }}$ ed. Yourdon Press, New York.

Zahran S (1998) Software Process Improvement - Practical Guidelines for Business Success. Addison-Wesley. 


\section{Appendix}

The initial software inspection process improvement pattern catalogue is presented in this appendix.

\section{Introduction}

This catalogue introduces seven patterns for inspection process improvement. Each pattern contains the following parts:

- Pattern name.

- Intent of the pattern describing briefly the purpose of the pattern.

- Problem description illustrating and giving examples of the problem to which the pattern can be applied.

- Symptoms briefly listing warning signs that suggest using the pattern.

- Context listing situations for which the pattern is particularly suitable.

- Solution listing the actions that need to be carried out to solve the problem.

- Consequences listing implications that utilizing the pattern may have in the organization.

It is recommended that one starts the search for a suitable pattern by looking at the symptoms sections of the pattern descriptions. The problem description gives more details about the motivation and background of a specific pattern. Actions listed in the solution section can be prioritized according to the needs of the situation. 


\section{The Patterns}

\section{Greed}

\section{Intent}

The intent of the Greed pattern is to upgrade the inspection process in order to uncover more defects during inspections.

\section{Problem}

Even though inspections are carried out frequently, they may produce poor results. Despite visible investment into the method its advantages remain unclear. Inspectors may consider the whole process a waste of time, and eventually management will think the same way, if no observable gains are achieved.

A certain degree of rigor is necessary in order to get the most out of the process. If this discipline is overlooked, or inspectors are not provided with adequate support to do their job, inspections will probably decrease productivity and motivation. A review is meaningful and efficient only if it reveals an adequate number of defects, and the defects discovered are not trivial.

The reasons for poor performance may vary. First, the artefact to be inspected or material related to the artefact may be unavailable or hard to obtain for the inspectors, or there may be shortcomings in the workflow of the process. Second, there might be an inadequate number of inspectors to read through the document, or they might not be provided with enough time for the review. Third, inspectors may not have up-to-date checklists and tools to support them through the process.

The following picture illustrates that insufficient resources or support can dilute the outcome of an inspection.

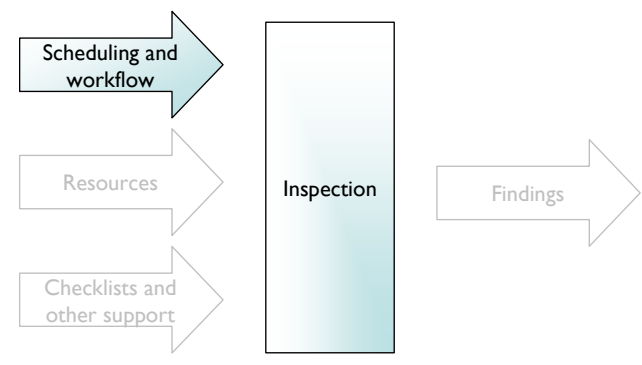

Using the Greed pattern should balance the process in order to get better results from the inspection. 


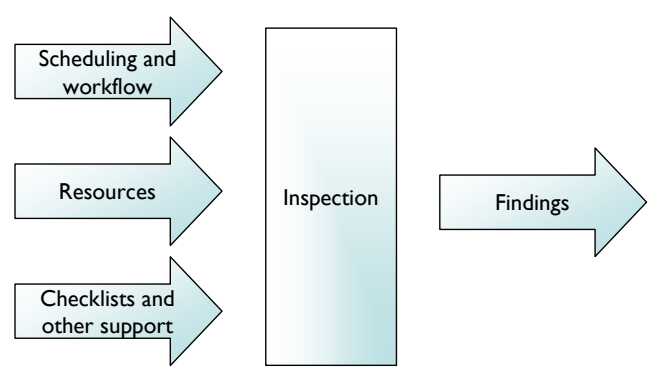

\section{Symptoms}

Symptoms that suggest that use of the Greed pattern would be beneficial include the following:

- Just a few errors are revealed in inspections.

- Only cosmetic errors are found in inspections.

- A lot of time is used to achieve poor results.

\section{Context}

Use the Greed pattern when you have recently installed the inspection process, or when the skill of using inspections has deteriorated, and the process does not perform efficiently enough. Inspection tasks may be seen as extra work and the benefits may seem vague. Inspections may be a normal procedure, but arranged and controlled carelessly by the QA group. If inspectors feel that their work is not supported or appreciated adequately, they will probably fail to discover real defects.

\section{Solution}

To approach the inspection process with the Greed pattern, upgrade the inspection process as follows:

1. Name a responsible person or persons to be the owner of the inspection process. The owner should pay special attention to reaching a common agreement (especially with the management) that inspections are to be planned, scheduled and resourced in every project. Consider adjusting the project plan document template so that inspections cannot be omitted.

2. Checklists have to be created or updated. Either the process owner has to take responsibility for checklist maintenance or another person has to be named for the task. It is handy to derive one checklist from one document template - a requirements checklist for requirements document template, for example. For code, use languagespecific coding conventions and such guides to create initial checklists. If possible, use historical data (search through a bug database, interview experienced developers) to find typical errors and write them into checklists.

3. Make artefact distribution more efficient. Study the feasibility of e-mail or other software tools. Ensure that checklists and source documents are really used by the 
inspectors. Observe their work and arrange training if possible. Ensure that checklists and source documents are distributed along with the artefact and that inspectors know how to use them.

4. Consider establishing specialist roles - for example, inspectors can focus on usability, interoperability or code-reusability aspects. Create separate sets of checklists from these specialist viewpoints. Train participants, if needed.

\section{Consequences}

Implementing actions listed in this pattern will have effects on activities P.5 (Maintain rules and checklists) and P.9 (Find issues in the artefact). Organizationally these improvements aim at making the inspection process more visible and owned. Actions defined in this pattern will strengthen the very essential practices of the process. Using this pattern may lead to modifications to the company's quality handbook, if planning and scheduling of inspection tasks are not already adequately addressed there. 


\section{Early Bird}

\section{Intent}

The intent of the Early Bird pattern is to upgrade the inspection process in order to reveal defects in earlier phases of the development cycle.

\section{Problem}

Inspections may be "plugged in" to the development process simply because "they are mandatory in our organization". It may seem that nobody is interested in the outcome of inspections, and even if there are significant outcomes, developers have already progressed to the next phase and cannot react to the feedback.

The following picture illustrates that if inspections are arranged separated from the development cycle, the results gained will be too late.

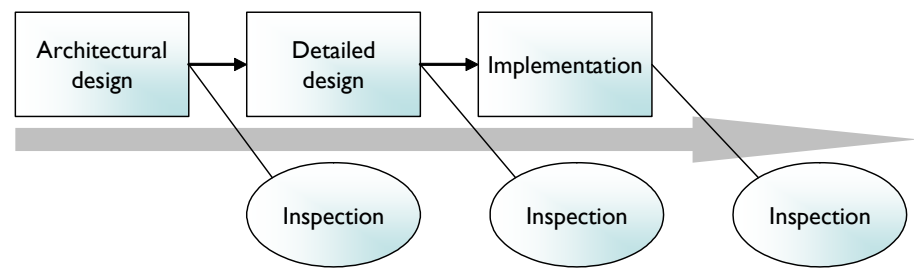

Using the Early Bird pattern should help in taking the inspection results into consideration in each phase of the development.

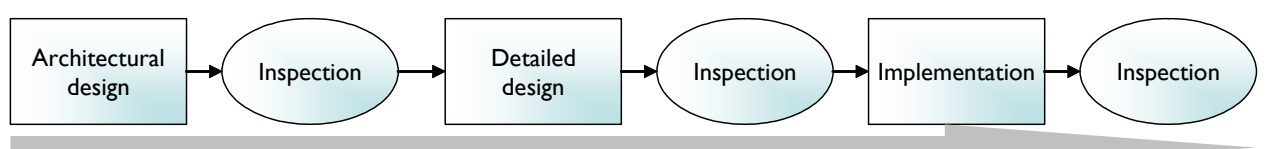

\section{Symptoms}

Symptoms that suggest that use of the Early Bird pattern would be beneficial include the following:

- Defects revealed in inspections are very serious and difficult to fix.

- A lot more errors are found in testing than in inspections.

- Product releases are postponed due to error-fixing activities.

\section{Context}

Use the Early Bird pattern when inspections are frequently arranged, but they are not controlled and their outcomes are not processed by the QA group. Inspectors and even project managers may feel that inspections are obligatory, extraneous tasks, and testing may be considered a more important and efficient method for quality assurance. 


\section{Solution}

To approach the inspection process with the Early Bird pattern, upgrade the inspection process as follows:

1. Include inspections in the quality handbook and ensure that inspections are included in all applicable phases of the development cycle. Make sure that resourcing is adequate. Motivating both the management and the people involved is beneficial. Bring up facts and figures about the efficiency of inspections.

2. Check that there are an adequate number of trained inspection leaders. Emphasize the importance and value of inspections to them, and remind them about their responsibility to plan and schedule inspections properly. Try to motivate leaders to perform review tasks, and consider rewarding the most diligent projects.

3. Ensure that there are working information channels between developers, projects and departments. When a piece of software or documentation is ready for inspection, it should be inspected as soon as possible. Also, ensure that tools utilized in inspection tasks work, and are available. Material distribution, reporting and other routine tasks should be straightforward. Take a look at checklists and make sure they are correct and available.

4. Check that the inspection process description includes a planning phase. Check that planning and scheduling is really done by inspection leaders and that each inspector has enough time to gain an understanding of the artefact.

5. Make sure that findings are reported immediately to the author of the inspected document. Ensure that the author has time to fix errors and that he or she gives rationales for the fixes. Start collecting experience data about error types.

\section{Consequences}

Typically, the company's quality handbook has to be updated when implementing this pattern. Integration of the inspection process and other development activities probably requires changes to the development process and work practices in place. 


\section{Substance}

\section{Intent}

The intent of the Substance pattern is to upgrade the inspection process in order to reveal more serious defects in inspections.

\section{Problem}

The following picture depicts a situation where an inspection uncovers only a couple of minor defects in the document under inspection.

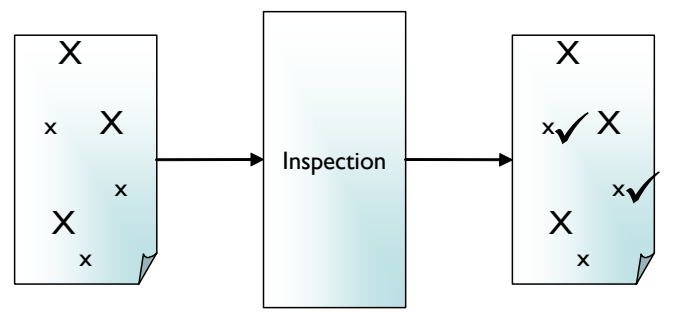

If inspectors do not have knowledge of or experience with the process, it is easiest to concentrate on typographic or other trivial defects and report those. When the purpose and goals of the review are unclear, people perhaps do not have the courage or interest to report any serious defects. To be meaningful, inspections should reveal an adequate number of severe defects.

Poor performance may be caused by insufficient training or inadequate checklist support. Checklists should focus attention on the most severe types of errors, and as the process evolves, checklists should be updated. In addition to checklists, it should be made clear how different types of source documents, categorization rules, specialist roles and other inspection guidelines should be used. All these supporting tools help in analysing the document under inspection more carefully and deeply.

Using the Substance pattern should help in revealing more serious issues.

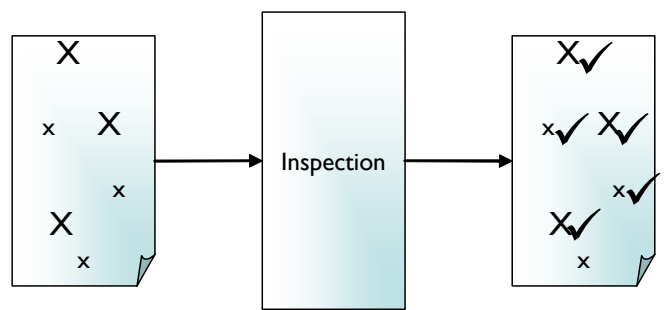




\section{Symptoms}

Symptoms that suggest that use of the Substance pattern would be beneficial include the following:

- Mainly cosmetic defects are reported in inspections.

- No significant modifications are made to inspected artefacts after review.

- The usefulness of inspections is unclear.

\section{Context}

Use the Substance pattern when inspections are arranged regularly, but produce insignificant results. Sometimes the constant absence of major findings may be wrongly interpreted as good quality, and usually inspections are just considered a waste of time, because lists of typographicla errors are the most visible outcome.

\section{Solution}

To approach the inspection process with the Substance pattern, upgrade the inspection process as follows:

1. The first thing to check is that the inspection process contains a categorization phase. Attention should be paid to analysis of the causes and types of errors, and inspectors should be aware that this analysis is as significant as revealing the defects. Also, check that in each inspection the participants really do carry out the initial categorization before reporting their findings. Ensure that each inspection leader requires this categorization when planning and arranging inspections.

2. Check that categorization guidelines are up-to-date and reasonable. Check that defect types correspond to the roles and rationales used in the development cycle. For instance, if the software is modularized into user interface, database and telecommunications components, it is natural to classify defects using that division. Other classification principles used must also be relevant. Severity classes that are too finely-grained may be hard to understand, for example. The usual major/minor classification is adequate in most situations. If the categorization system is too complex or too simple, the inspectors will probably get frustrated and will not use it correctly.

3. Update checklists. Irrelevant lists and list items should be dropped. Try to exploit experience data about the usage of checklists. Compare checklists to software engineering rules and guidelines used in the company. Consider defining roles, perspectives and scenarios based on different development aspects: designer, tester, database, communications, etc.

4. Take advantage of typo lists and other forms that can be used to report non-major findings. This should help inspectors concentrate on more important issues, and emphasizes the reporting of at least some major defects.

5. Train the people involved in inspections. Write a tutorial and make it available. Make sure that inspection leaders have special training. Emphasize the importance of reviews to all inspectors, leaders, and project managers.

6. Check that the material to be inspected is cursorily checked by the inspection leader to ensure the initial quality of the work product. 


\section{Consequences}

Utilizing the Substance pattern is not expected to increase the number of defects found. In fact, the total number of reported defects may decrease, as preconditions for inspections are checked more rigorously and inspectors are encouraged to search for more serious faults. Thus, the aim is to reveal more severe issues. Introducing checklists, typo lists and other inspector aids may also initiate tool upgrade and development. It may be beneficial to look at the Comfort pattern, if a considerable amount of effort is needed for such upgrading.

Training must also be arranged to ensure that people understand the meaning and usage of checklists and categorization tools. This training requires a few hours of work and should not require inspectors to sacrifice their free time. 


\section{Comfort}

\section{Intent}

The intent of the Comfort pattern is to upgrade the inspection process in order to make the process easier to carry out and more flexible.

\section{Problem}

It is not always clear what people should do in inspections and when they should do it, especially if the process has only recently been introduced in the organization. It may seem that there are an enormous number of forms to fill in, papers to print and meetings to attend. The situation may be even more difficult from the moderator's viewpoint: which forms should be delivered to whom, how should the material be distributed, when can meetings be arranged. The following picture depicts such a situation. The review is seen as having much larger importance than the artefact itself.

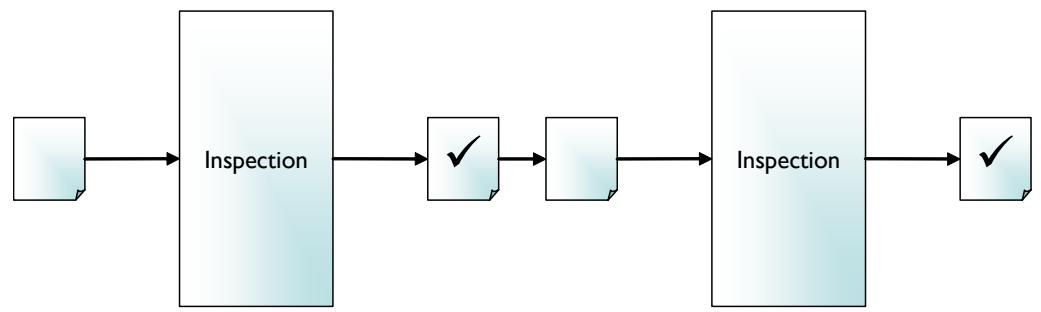

The Comfort pattern tries to re-arrange the inspection in order to make the procedure effortless, or even automate certain parts of it.

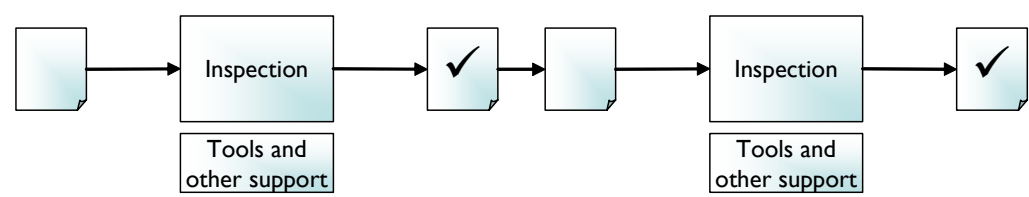

\section{Symptoms}

Symptoms that suggest that use of the Comfort pattern would be beneficial include the following:

- An inspection takes a lot of time.

- Inspectors are unhappy with the effort they have to put into review tasks.

- There are difficulties in arranging inspections due to scheduling and resourcing problems. 


\section{Context}

Use the Comfort pattern when inspections are burdensome.

\section{Solution}

To approach the inspection process with the Comfort pattern, upgrade the inspection process as follows:

1. Start by estimating the need for computerized inspection tools. If the inspection team is distributed and meetings are difficult to arrange, tools are especially useful for material distribution and communication.

2. Perform a feasibility study for a selection of inspection tools. Assess the need to adjust working methods (e.g., changing authoring tools, the need for a common document format). In a very small organization, a set of spreadsheet or word processing templates is an adequate tool.

3. Install tools and establish resources for maintaining them.

4. Even though the tools may be simple, such as forms and templates, pay attention to the standardization of tasks and documentation. Ensure that material is always delivered to inspectors in a similar way. Ensure that appropriate source documents and checklists are always bundled and delivered along with the document under inspection.

5. Consider establishing tools or templates for online commenting and reporting of findings, and macros to calculate basic metrics automatically. Try to automate reporting. For example, you can ask inspectors to report their findings in the form of a spreadsheet which automatically creates a graph of major findings.

6. If there are tools in use, check that time is not wasted in document format conversions, filling in obvious data fields, copying data from one form to another, etc. Update the tools, if possible. Set responsibilities to prevent several people doing the same thing.

7. Arrange training concerning tools, templates and the process.

\section{Consequences}

Typically, using the Comfort pattern introduces new tools into the work process. This, in turn, involves training for these tools. Without the proper training, the tools will not be used and they will eventually be forgotten. The need for tools and implementing (or purchasing) them will probably require a project of its own. 


\section{Promotion}

\section{Intent}

The intent of the Promotion pattern is to promote the inspection process so that inspections are carried out more often and for a larger number of projects.

\section{Problem}

Particularly when the inspection process is poorly defined in the quality handbook, or people working in real software projects are not motivated to take part in reviews, inspections may be dropped almost completely. If there is an organizational obligation to carry out inspections, they may be arranged every now and then in some less important phases of the projects. Another reason for rarely performed inspections may be a lack of organizational commitment. Even though certain project managers may see the value of inspections and arrange them in their projects, it requires management-level commitment to make inspections an established activity.

The following picture gives an example of a situation where documentation of each development phase is inspected just partially. The documents for review are selected randomly.

Architectural design

Detailed design

Implementation

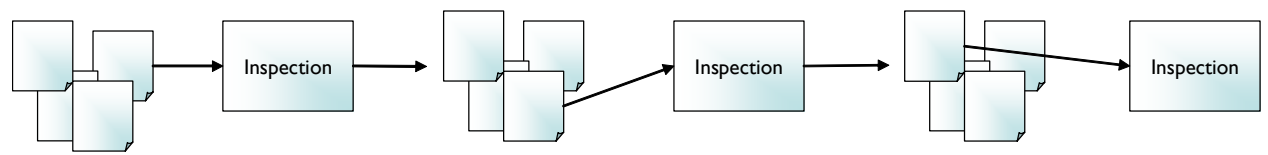

The Promotion pattern tries to create a culture where all documentation relevant to software development is inspected.

Architectural design

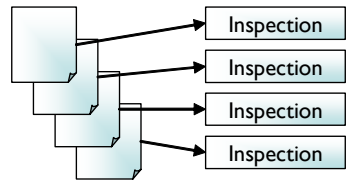

Detailed design

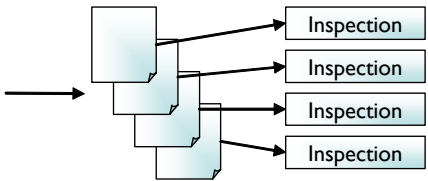

Implementation

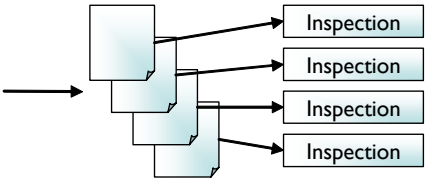

\section{Symptoms}

Symptoms that suggest that use of the Promotion pattern would be beneficial include the following:

- Inspections are not performed in every project.

- Inspections are not considered useful. 
- Authors are reluctant to deliver material for inspections.

- Inspections are seen as personal criticism.

\section{Context}

Use the Promotion pattern when inspections are not considered important.

\section{Solution}

To approach the inspection process with the Promotion pattern, upgrade the inspection process as follows:

1. The first step is to ensure that inspection is a company policy. Include the inspection process in the quality handbook, if it is not already there. Promote the process to management and request adequate resources to administrate and improve the inspection process. If inspections are already listed in the quality handbook, identify their weakest areas and create a concrete schedule for improving the performance of the process. Ask people who have worked as moderators what is wrong with the process. You can also consult an outside expert.

2. The initial performance is best ensured by establishing a minimum set of metrics. The data gathered can also be used to motivate management. Time used, number of defects found and severity of defects are the very basic measures. Collect them systematically. Study the performance of each inspection step too, and ask for improvement suggestions and feedback about the process itself. Recording the lessons learned and reporting them actively to the management as well as to the inspectors and project managers is crucial.

3. Ensure that there are enough trained moderators. Arrange training for inspectors too. Make sure that the process, templates, metrics and tools are well-known by everybody. Good knowledge usually motivates the people involved.

4. Consider rewarding project workers who take inspections seriously and do the most diligent job.

\section{Consequences}

A little organization-wide effort is needed to advertise the inspection process and to motivate inspectors to do a good job in every inspection. Management support and "inspection champions" are very important in achieving wide-spread use of the process. Everybody has to feel that inspections really have a significant and direct impact on product quality.

The whole development process is expected to change when utilizing the Promotion pattern. Inspections are made visible in the software process, and the general attitude towards reviews has to be positive and encouraging. 


\section{Wisdom}

\section{Intent}

The intent of the Wisdom pattern is to improve the effectiveness of inspections by enhancing their understandability.

\section{Problem}

Recording best practices concerning review tasks is the first step towards starting continuous improvement of the process. If the results of each inspection are not systematically recorded and analysed, reviewing documents has to be started from scratch every time. There is a vast amount of data involved in inspections that can be utilized throughout development. It is in inspections that the most typical types of errors are revealed, and a great quantity of metrics can be gathered. Collecting the lessons learned and really learning them may improve the development process greatly.

The following figure depicts a situation where inspections do not take advantage of any specific knowledge base.
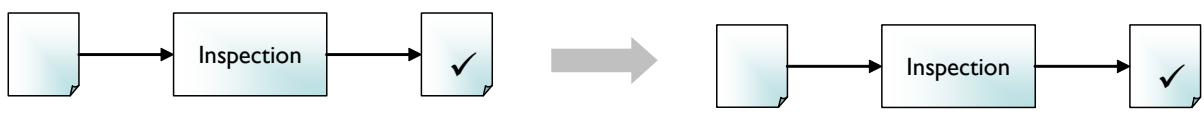

With the Wisdom pattern, the lessons learned from each inspection are recorded and referred to when arranging the next inspection.

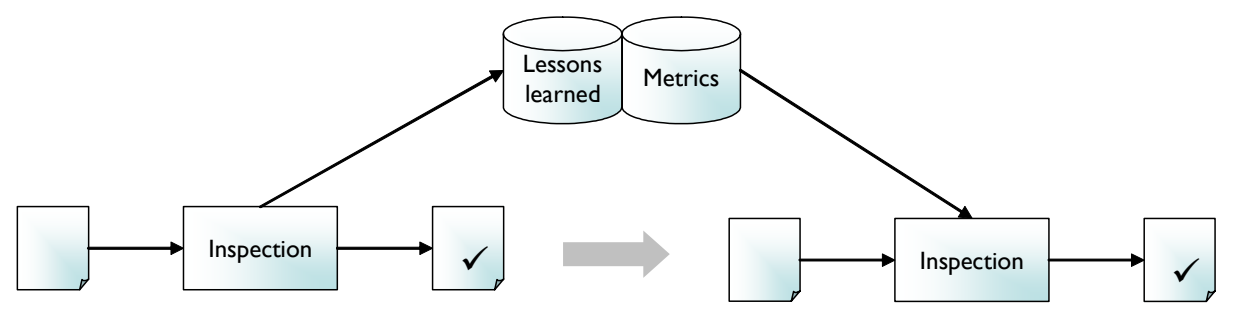

\section{Symptoms}

Symptoms that suggest that use of the Wisdom pattern would be beneficial include the following:

- The usefulness of inspections is unclear and they are seen as an organizational duty.

- In every inspection, the same sort of problems and defects are encountered.

- Only minor defects are reported. 


\section{Context}

Use the Wisdom pattern when inspections have been introduced into the organization some time ago, but still do not produce significant results. If there are frequent attempts to improve the effectiveness of inspections, and recognized problems reoccur every time, try using the Wisdom pattern. If inspectors and inspection leaders confront the same problems over and over again, this pattern may help in getting the process onto the next level.

\section{Solution}

To approach the inspection process with the Wisdom pattern, upgrade the inspection process as follows:

1. Define an appropriate set of metrics for both process quality and artefact quality. The minimum set is: time used to review a document, number of issues found, and severity of issues. You can refer to inspection literature to define more metrics. Ensure that the people participating in inspections interpret the metrics similarly. This is especially challenging with severity measures. Make this knowledge base available to everybody involved in inspections.

2. Make inspection results visible by establishing a standard reporting practice to inform the management, inspection leaders and individual inspectors. Management is mostly interested in inspection efficiency, inspection leaders need data for planning and scheduling inspections, and inspectors may be interested in defect types and severities.

3. Pay special attention to categorization. In addition to the typical major/minor classification, consider other types of classes, such as defect source (requirements, specification, etc), structure-based classification (user interface, database, etc), or lifecycle-based classification (new feature, updated source code, maintained code, etc). Make sure that the categorization is understood and known by everybody. If feedback suggests that the categorization does not work, change it.

4. Perform systematic follow-up. Give the author adequate time and resources to fix defects. Keep records of fixes, and generate reports of the most typical errors to help others avoid repeating them.

\section{Consequences}

Typically, activities A.6, A.10 and A.11 are affected by the Wisdom pattern. Using this pattern may cause a considerable initial cost, as establishing data collection scheme and metrics, and analysing the data requires a basic set of tools. Even if these tools are spreadsheet forms, they still involve specification and implementation effort. Once the correct tools and metrics are in place, inspections should run smoothly and without extra work.

Using the Wisdom pattern gives an excellent opportunity to make use of the data collected in other development processes as well. It may be possible at the same time to implement a metrics programme involving the whole development cycle. Thus, using the solution described in the Wisdom pattern may bring even more considerable benefits. 


\section{Precision}

\section{Intent}

The intent of the Precision pattern is to improve the effectiveness of inspections by improving the structure of the process.

\section{Problem}

The following figure illustrates an inspection process that is poorly organized. When review tasks are carried out only partially, the process will probably produce limited results.

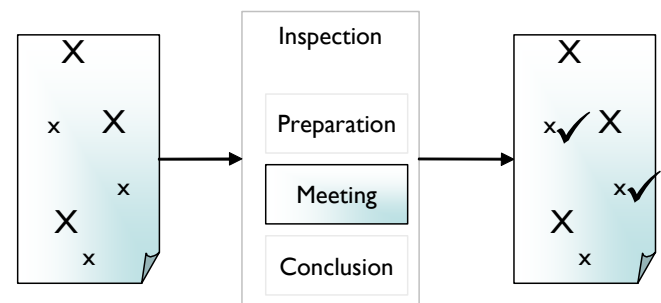

Perhaps the most typical reason for bad performance is that inspections are arranged in an ad hoc style. Lack of organizational commitment to the inspection process, or inadequate understanding of its significance may result in a compulsory, uncontrolled process, where nobody seems to be interested in its results. Inspections have to be planned. Planning starts from the organizational level and leads to a single inspection leader. The tasks and purposes of the method have to be clear to everybody.

Proper planning requires a clearly defined process, which means that inspections have to be declared a common policy for every project in the quality handbook. In addition to clear definition, the process has to be carried out properly. To enable that, an adequate inspection infrastructure has to be established in the form of checklists, categorization and so on.

With the Precision pattern, the structure and the rigidity of the process are improved, resulting in a more effective review process.

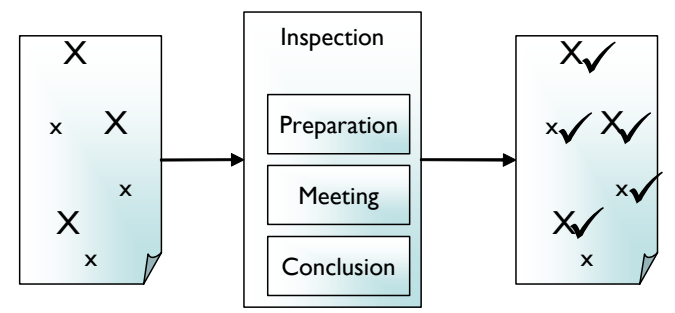




\section{Symptoms}

Symptoms that suggest that use of the Precision pattern would be beneficial include the following:

- Artefacts are of poor quality even after going through inspections.

- Lots of inspected artefacts are "approved with changes".

- Inspections are postponed or rescheduled.

\section{Context}

Use the Precision pattern when inspections are practiced, but do not seem to produce relevant results. Due to their pointlessness, inspections are not taken seriously. There may be a complete process description for inspection, but some parts of the process may be omitted or replaced with light-weight versions.

\section{Solution}

To approach the inspection process with the Precision pattern, upgrade the inspection process as follows:

1. Check the inspection process definition in the quality handbook. Ensure that inspections are required in each phase of the development. Ensure that motivation for reviewing documents is given and examples are given to demonstrate the value of the process. Check that the defined process includes an adequate number of phases, in order to reveal as many defects as possible.

2. Motivate the organization and the people. Show the management facts and figures about the efficiency of inspections. Organize a workshop for developers. Emphasize that inspections are not to judge individual developers but to support them.

3. Study the efficiency of inspections. Identify the weakest points: is enough time spent in preparation? Is categorization relevant? Are checklists up-to-date? Ask for feedback from inspection leaders and inspectors to identify the most frustrating parts of the process. You can also consult an outside expert to get an assessment of the quality of the process.

4. Create an inspection infrastructure. Start recording the lessons learned, and establish a suitable set of metrics for the collection of inspection data. At the very least, time, number of defects and severity should be measured systematically. Clearly define entry and exit criteria for documents and ensure that these criteria are used.

5. Pay attention to the follow-up. The author has to be given enough time to fix errors. Furthermore, the rationales for defect elimination can be requested and recorded, in order to transfer software development knowledge and best practices.

\section{Consequences}

The Precision pattern typically affects activities A.1, A.2, A.7, A.11 and A.12. These activities are mostly related to the organization and management of the process. At the same time, these activities are those that are very easily forgotten and which, perhaps, deteriorate over time.

Utilizing the Precision pattern may cause a slight increase in resource needs, because it involves tasks that have in all likelihood previously been considered unnecessary. 
Checking preconditions and postconditions is quite effortless, but if there are problems with inspection process definition and ownership, more resources will be needed.

The Precision pattern does not greatly affect other development practices, as it is assumed that it is the inspection part of the overall development that is being carried out carelessly. 